\title{
Hierarchy spectrum of SM fermions: from top quark to electron neutrino
}

\author{
She-Sheng Xue \\ ICRANet, \\ Piazza della Repubblica 10, 65122 Pescara, Italy \\ Physics Department, Sapienza University of Rome, \\ Piazzale Aldo Moro 5, 00185 Roma, Italy \\ E-mail: xue@icra.it
}

ABSTRACT: In the SM gauge symmetries and fermion content of neutrinos, charged leptons and quarks, we study the effective four-fermion operators of Einstein-Cartan type and their contributions to the Schwinger-Dyson equations of fermion self-energy functions. The study is motivated by the speculation that these four-fermion operators are probably originated due to the quantum gravity, which provides the natural regularization for chiral-symmetric gauge field theories. In the chiral-gauge symmetry breaking phase, as to achieve the energetically favorable ground state, only the top-quark mass is generated via the spontaneous symmetry breaking, and other fermion masses are generated via the explicit symmetry breaking induced by the top-quark mass, four-fermion interactions and fermion-flavor mixing matrices. A phase transition from the symmetry breaking phase to the chiral-gauge symmetric phase at $\mathrm{TeV}$ scale occurs and the drastically fine-tuning problem can be resolved. In the infrared fixed-point domain of the four-fermion coupling for the SM at low energies, we qualitatively obtain the hierarchy patterns of the SM fermion Dirac masses, Yukawa couplings and family-flavor mixing matrices with three additional right-handed neutrinos $\nu_{R}^{f}$. Large Majorana masses and lepton-number symmetry breaking are originated by the four-fermion interactions among $\nu_{R}^{f}$ and their left-handed conjugated fields $\nu_{R}^{f c}$. Light masses of gauged Majorana neutrinos in the normal hierarchy $\left(10^{-5}-10^{-2} \mathrm{eV}\right)$ are obtained consistently with neutrino oscillations. We present some discussions on the composite Higgs phenomenology and forward-backward asymmetry of $t \bar{t}$-production, as well as remarks on the candidates of light and heavy dark matter particles (fermions, scalar and pseudoscalar bosons).

Keywords: Quark Masses and SM Parameters, Spontaneous Symmetry Breaking, Technicolor and Composite Models

ARXIV EPRINT: 1605.01266 


\section{Contents}

1 Introduction 1

2 Four-fermion operators beyond the SM 4

2.1 Regularization and quantum gravity 4

2.2 Einstein-Cartan theory with the SM gauge symmetries and fermion content 5

$\begin{array}{lll}2.3 & \text { SM gauge-symmetric four-fermion operators } & 6\end{array}$

$\begin{array}{lll}2.4 & \text { Four-fermion operators of quark-lepton interactions } & 7\end{array}$

3 Gauge vs mass eigenstates in fermion-family space $\quad 7$

$\begin{array}{lll}3.1 \text { Quark sector } & 7\end{array}$

$\begin{array}{llr}3.2 & \text { Lepton sector } & 9\end{array}$

$\begin{array}{ll}3.3 \text { Quark-lepton interaction sector } & 10\end{array}$

4 Spontaneous symmetry breaking $\quad 11$

4.1 The IR fixed-point domain and only top-quark mass generated via the SSB 11

$\begin{array}{ll}4.2 \text { The }\langle\bar{t} t\rangle \text {-condensate model } & 12\end{array}$

4.2.1 The scaling region of the IR-stable fixed point 13

4.2.2 Experimental indications of composite Higgs boson? 14

5 Origins of explicit symmetry breaking $\quad \mathbf{1 5}$

$\begin{array}{ll}5.1 \text { Quark-lepton interactions } & 15\end{array}$

$\begin{array}{lll}5.2 W^{ \pm} \text {-boson coupling to right-handed fermions } & 18\end{array}$

6 Schwinger-Dyson equations for fermion self-energy functions 18

$\begin{array}{ll}\text { 6.1 Chiral symmetry-breaking terms in SD equations } & 19\end{array}$

6.2 Twelve coupled SD equations for SM quark and lepton masses 19

$\begin{array}{lll}6.3 & \text { Realistic massive solutions } & 20\end{array}$

7 The hierarchy spectrum of SM fermion masses $\quad 21$

$\begin{array}{ll}7.1 \text { The third fermion family } & 21\end{array}$

7.1.1 Approximate fermion mass-gap equations for the third family 21

7.1.2 Fermion masses and running Yukawa couplings 22

$\begin{array}{ll}7.2 & \text { The second fermion family } 23\end{array}$

7.2.1 Approximate fermion mass-gap equations of the second family 24

7.2.2 Running fermion masses and Yukawa couplings 25

$\begin{array}{ll}7.3 & \text { The first fermion family } \\ & 26\end{array}$

7.3.1 Approximate mass-gap equations of the first fermion family 26

$\begin{array}{ll}\text { 7.3.2 Running fermion masses and Yukawa couplings } & 27\end{array}$

$\begin{array}{lll}7.4 & \text { Summary and discussion } & 28\end{array}$ 
8.1 Spontaneous symmetry breaking of $U_{\text {lepton }}(1)$ symmetry 30

8.2 Gauged and sterile Majorana neutrino masses 31

$\begin{array}{lll}\text { 8.3 Flavor oscillations of gauged Majorana neutrinos } & 32\end{array}$

8.4 Flavor oscillations of sterile Majorana neutrinos 34

8.5 Oscillations between gauged and sterile Majorana neutrinos 35

$9 \quad$ A summary and some remarks $\quad 36$

9.1 SM fermion Dirac masses and Yukawa couplings 36

$\begin{array}{lll}9.2 & \text { Neutrinos and dark-matter particles } & 37\end{array}$

\section{Introduction}

The parity-violating (chiral) gauge symmetries and spontaneous/explicit breaking of these symmetries for the hierarchy pattern of fermion masses have been at the center of a conceptual elaboration that has played a major role in donating to mankind the beauty of the Standard Model (SM) for fundamental particle physics. On the one hand the composite Higgs-boson model or the Nambu-Jona-Lasinio (NJL) [1] with effective four-fermion operators, and on the other the phenomenological model [2-7] of the elementary Higgs boson, they are effectively equivalent for the SM at low energies and provide an elegant and simple description for the chiral electroweak symmetry breaking and intermediate gauge boson masses. The experimental measurements of Higgs-boson mass $126 \mathrm{GeV}$ [8, 9] and top-quark mass $173 \mathrm{GeV}[10,11]$, as well as the other SM fermion masses and family-mixing angles, in particular neutrino oscillations, begin to shed light on this most elusive and fascinating arena of fundamental particle physics.

The patterns of the SM fermion masses and family-mixing matrices are equally fundamental, and closely related. Since Gatto et al. [12] tried to find the relation between the Cabibbo mixing angle and light-quark masses, the tremendous effort and many models have been made to study the relation of the SM fermion masses and family-mixing matrices from the phenomenological and/or theoretical view points [13-74], where the references are too many to be completely listed. In literature the most of effort based on phenomenological models assuming a particular texture in the original fermion-mass matrices in quark and/or lepton sectors to find the fermion-family mixing matrices as functions of observed fermion masses, i.e., the eigenvalues of the original fermion-mass matrices. Whereas some other models try to find the relations of fermion masses and family-mixing matrices on the basis of theoretically model-building approaches, for example, the left-right symmetric scenario $[12-16]$ and $[23,49]$, string theory phenomenology $[50,51]$ or the scenario of effective vector-like $W^{ \pm}$-coupling at high energies $[32,33]$. In the model-independent approach, the fermion-mass matrices with different null matrix elements (texture zeros) are considered to find the relations of fermion mass and mixing patterns [52-57]. The gauge symmetries of grand unification theories, like $\mathrm{SO}(10)$-theory, and/or the fermion-flavor symmetries, like 
horizontal or family discrete symmetry, are adopted to find non-trivial relations of fermion mass and mixing patterns [22-25, 57-62] and [68-74]. As the precision measurements for neutrino oscillations are progressing $[47,63,64]$, the study of neutrino mass pattern and lepton-flavor mixing becomes vigorously crucial [65-67].

In this article, we approach to this long-standing problem by considering effective four-fermion operators in the framework of the SM gauge symmetries and fermion content: neutrinos, charged leptons and quarks. In order to accommodate high-dimensional operators of fermion fields in the SM-framework of a well-defined quantum field theory at the high-energy scale $\Lambda$, it is essential and necessary to study: (i) what physics beyond the SM at the scale $\Lambda$ explains the origin of these operators; (ii) which dynamics of these operators undergoes in terms of their dimensional couplings (e.g., $G$, see below) and energy scale $\mu$; (iii) associating to these dynamics, where infrared (IR) and ultraviolet (UV) stable fixed points of these couplings locate and what characteristic energy scales are; (iv) in the IR-domain and UV-domain (scaling regions) of these stable IR and UV fixed points, which operators become physically relevant (effectively dimension-4) and renormalizable following renormalization group (RG) equations (scaling laws), and other irrelevant operators are suppressed by the cutoff at least $\mathcal{O}\left(\Lambda^{-2}\right)$.

We briefly recall that the strong technicolor dynamics of extended gauge theories at the $\mathrm{TeV}$ scale was invoked [75-80] to have a natural scheme incorporating the four-fermion operator

$$
L=L_{\text {kinetic }}+G\left(\bar{\psi}_{L}^{i a} t_{R a}\right)\left(\bar{t}_{R}^{b} \psi_{\text {Lib }}\right),
$$

of Bardeen, Hill and Lindner (BHL) $\langle\bar{t} t\rangle$-condensate model [81] in the context of a welldefined quantum field theory at the high-energy scale $\Lambda$. The four-fermion operator (1.1) undergoes the spontaneous symmetry breaking (SSB) dynamics responsible for the generation of top-quark and Higgs-boson masses in the domain of IR-stable fixed point $G_{c}$ (critical value associated with the SSB) and characteristic energy scale (vev) $v \approx 239.5 \mathrm{GeV}$. The analysis of this composite Higgs boson model shows [81] that eq. (1.1) effectively becomes a bilinear and renormalizable Lagrangian following RG equations, together with the composite Goldstone modes for the longitudinal components of massive $W^{ \pm}$and $Z^{0}$ gauge bosons, and the composite scalar for the Higgs boson. The low-energy SM physics, including the values of top-quark and Higgs-boson masses, was supposed to be achieved by the RG-equations in the domain of the IR-stable fixed point [78-81, 85]. On the other hand, the relevant operator (1.1) can be constructed on the basis of the SM phenomenology at low-energies. It was suggested ([81-83, 85]; the SU(3)-extension of their work in chapter 26 of the textbook [84]) that the symmetry breakdown of the SM could be a dynamical mechanism of the NJL type that intimately involves the top quark at the high-energy scale $\Lambda$, since then, many models based on this idea have been studied [86, 87].

Nowadays, the known top-quark and Higgs boson masses completely determine the boundary conditions of the RG equations for the top-quark Yukawa coupling $\bar{g}_{t}(\mu)$ and Higgs-boson quartic coupling $\tilde{\lambda}(\mu)$ in the composite Higgs boson model (1.1). Using the experimental values of top-quark and Higgs boson masses, we obtained [88, 89] the unique solutions $\bar{g}_{t}(\mu)$ and $\tilde{\lambda}(\mu)$ to these RG equations, provided the appropriate non-vanishing 
form-factor $\tilde{Z}_{H}(\mu)=1 / \bar{g}_{t}^{2}(\mu)$ of the composite Higgs boson at the energy-scale $\mathcal{E} \sim \mathrm{TeV}$, where the effective quartic coupling $\tilde{\lambda}(\mu)$ of composite Higgs bosons vanishes.

The form-factor of composite Higgs boson $H \sim(\bar{\psi} \psi)$ is finite and does not vanish in the SSB phase (composite Higgs phase for small $G \gtrsim G_{c}$ ), indicating that the tightly bound composite Higges particle behaves as if an elementary particle. On the other hand, due to large four-fermion coupling $G$, massive composite fermions $\Psi \sim(H \psi)$ are formed by combining a composite Higgs boson $H$ with an elementary fermion $\psi$ in the symmetric phase where the SM gauge symmetries are exactly preserved [90-94]. This indicates that a second-order phase transition from the SSB phase to the SM gauge symmetric phase takes place at the critical point $G_{\text {crit }}>G_{c}$. In addition the effective quartic coupling of composite Higgs bosons vanishing at $\mathcal{E} \sim \mathrm{TeV}$ scales indicates the characteristic energy scale of such phase transition. The energy scale $\mathcal{E}$ is much lower than the cutoff scale $\Lambda(\mathcal{E} \ll \Lambda)$ so that the drastically fine-tuning (hierarchy) problem that fermion masses $m_{f} \ll \Lambda$ or the pseudoscalar decay constant $f_{\pi} \ll \Lambda$ can be possibly avoided by the replacements $m_{f}<\mathcal{E}$ or the pseudoscalar decay constant $f_{\pi}<\mathcal{E}$ [88].

In ref. [97], after a short review that recalls and explains the quantum-gravity origin of four-fermion operators at the cutoff $\Lambda$, the BHL $\langle\bar{t} t\rangle$-condensate model and the SSB, we show that due to four-fermion operators (i) there are the SM gauge symmetric vertexes of quark-lepton interactions; (ii) the one-particle-irreducible (1PI) vertex-function of $W^{ \pm}$-boson coupling becomes approximately vector-like at $\mathrm{TeV}$ scale. Both interacting vertexes contribute the explicit symmetry breaking (ESB) terms to the Schwinger-Dyson (SD) equations of fermion self-energy functions. As a result, once the top-quark mass is generated via the SSB, the masses of third fermion family $\left(\nu_{\tau}, \tau, b\right)$ are generated by the ESB via quark-lepton interactions and $W^{ \pm}$-boson vector-like coupling. Within the third fermion family, we qualitatively study the hierarchy of fermion masses and effective Yukawa couplings in terms of the top-quark mass and Yukawa coupling [97].

In this article, we generalize this study into three fermion families of the SM by taking into account the flavor mixing of three fermion families. Such flavor mixing inevitably introduces the 1PI vertex-functions of quark-lepton interactions and approximately vectorlike $W^{ \pm}$-boson coupling among three fermion families at $\mathrm{TeV}$ scale. As a consequence, these 1PI vertex-functions introduce the ESB terms into the SD-equations of the fermion self-energy functions for all SM fermions in three families. Once the top-quark mass is generated via the SSB, all other SM fermions acquire their masses via the ESB terms by (i) four-fermion interactions among fermion flavors via family mixing matrices; (ii) the $W^{ \pm}$-boson coupling among fermion flavors via the CKM or PMNS mixing matrix. The latter is dominate particularly for light quarks and leptons. As a result, we quatitatively obtain the hierarchy patterns of the SM fermion masses and family-mixing matrices, and all fermion masses and Yukawa couplings are functions of the top-quark mass and Yukawa coupling. Neutrino masses will separately be studied in the last part of the article, for its peculiarity.

This lengthy article is organized as follow. In section 2, we give an argument why fourfermion operators should be present in an effective Lagrangian at the high-energy cutoff at which the quantum gravity introduces a natural regulator for chiral gauge theories. In 
the framework of the SM gauge symmetries and fermion content, we discuss four-fermion operators, including quark-lepton interactions. In section 3, we describe fermion-flavor mixing matrices in lepton and quark sectors, as well as quark-lepton interaction sector. In section 4, we give a brief recall that the SSB is responsible only for the top-quark and Higgs boson masses, whose values determine the unique solution to the RG equations for the topquark Yukawa and composite Higgs quartic couplings. In sections 5 and 6, we discuss the ESB terms and massive solutions of SD equations of other SM fermions. In section 7, we qualitatively present the hierarchy patterns of the SM fermions and fermion-flavor mixing matrices. In the last section 8, we focus on the discussions of gauged and sterile neutrinos of Dirac or Majorana type, and their masses, mixing and oscillation. A brief summary and some remarks are given at the end of the article. ${ }^{1}$

\section{Four-fermion operators beyond the SM}

\subsection{Regularization and quantum gravity}

Up to now the theoretical and experimental studies tell us the chiral gauge-field interactions to fermions in the lepton-quark family that is replicated three times and mixed. The spontaneous breaking of these chiral gauge symmetries and generating of fermion masses are made by the Higgs field sector. In the IR-fixed-point domain of weak four-fermion coupling or equivalently weak Yukawa coupling, the SM Lagrangian with all relevant operators (parametrizations) is realized and behaves an effective and renormalizable field theory in low energies. To achieve these SM relevant operators, a finite field theory of chiral-gauge interactions should be well-defined by including the quantum gravity that naturally provides a space-time regularization (UV cutoff). As an example, the finite superstring theory is proposed by postulating that instead of a simple space-time point, the fundamental space-time "constituents" is a space-time "string". The Planck scale is a plausible cut-off, at which all principle and symmetries are fully respect by gauge fields and particle spectra, fermions and bosons.

In this article, we do not discuss how a fundamental theory at the Planck scale induces high-dimensional operators. Instead, as a postulation or motivation, we argue the presence of at least four-fermion operators beyond the SM from the following point view. A well-defined quantum field theory for the SM Lagrangian requires a natural regularization (UV cutoff $\Lambda$ ) fully preserving the SM chiral-gauge symmetry. The quantum gravity naturally provides a such regularization of discrete space-time with the minimal length $\tilde{a} \approx 1.2 a_{\mathrm{pl}}$ [98-100], where the Planck length $a_{\mathrm{pl}} \sim 10^{-33} \mathrm{~cm}$ and scale $\Lambda_{\mathrm{pl}}=\pi / a_{\mathrm{pl}} \sim 10^{19} \mathrm{GeV}$. However, the no-go theorem [101-104] tells us that there is no any consistent way to regularize the SM bilinear fermion Lagrangian to exactly preserve the SM chiral-gauge symmetries, which must be explicitly broken at the scale of fundamental space-time cutoff $\tilde{a}$. This implies that the natural quantum-gravity regularization for the SM should lead us to consider at least dimension-6 four-fermion operators originated

\footnotetext{
${ }^{1}$ More discussions on the experimental aspects of this scenario can be found in the refs. S.-S. Xue [130] and in the last section of [97] and references therein.
} 
from quantum gravity effects at short distances. ${ }^{2}$ As a model, we adopt the four-fermion operators of the torsion-free Einstein-Cartan Lagrangian within the framework of the SM fermion content and gauge symmetries. We stress that a fundamental theory at the UV cutoff is still unknown.

\subsection{Einstein-Cartan theory with the SM gauge symmetries and fermion con- tent}

The Lagrangian of torsion-free Einstein-Cartan (EC) theory reads,

$$
\mathcal{L}_{\mathrm{EC}}(e, \omega, \psi)=\mathcal{L}_{\mathrm{EC}}(e, \omega)+\bar{\psi} e^{\mu} \mathcal{D}_{\mu} \psi+G J^{d} J_{d}
$$

where the gravitational Lagrangian $\mathcal{L}_{\mathrm{EC}}=\mathcal{L}_{\mathrm{EC}}(e, \omega)$, tetrad field $e_{\mu}(x)=e_{\mu}{ }^{a}(x) \gamma_{a}$, spinconnection field $\omega_{\mu}(x)=\omega_{\mu}^{a b}(x) \sigma_{a b}$, the covariant derivative $\mathcal{D}_{\mu}=\partial_{\mu}-i g \omega_{\mu}$ and the axial current $J^{d}=\bar{\psi} \gamma^{d} \gamma^{5} \psi$ of massless fermion fields. The four-fermion coupling $G$ relates to the gravitation-fermion gauge coupling $g$ and fundamental space-time cutoff $\tilde{a}$.

Within the SM fermion content, we consider massless left- and right-handed Weyl fermions $\psi_{L}^{f}$ and $\psi_{R}^{f}$ carrying quantum numbers of the SM symmetries, as well as three right-handed Weyl sterile neutrinos $\nu_{R}^{f}$ and their left-handed conjugated fields $\nu_{R}^{f c}=$ $i \gamma_{2}\left(\nu_{R}\right)^{*}$, where " $f$ " is the fermion-family index. Analogously to the EC theory (2.1), we obtain a torsion-free, diffeomorphism and local gauge-invariant Lagrangian

$$
\begin{aligned}
\mathcal{L}= & \mathcal{L}_{\mathrm{EC}}(e, \omega)+\sum_{f} \bar{\psi}_{L, R}^{f} e^{\mu} \mathcal{D}_{\mu} \psi_{L, R}^{f}+\sum_{f} \bar{\nu}_{R}^{f c} e^{\mu} \mathcal{D}_{\mu} \nu_{R}^{f c} \\
& +G\left(J_{L}^{\mu} J_{L, \mu}+J_{R}^{\mu} J_{R, \mu}+2 J_{L}^{\mu} J_{R, \mu}\right)+G\left(j_{L}^{\mu} j_{L, \mu}+2 J_{L}^{\mu} j_{L, \mu}+2 J_{R}^{\mu} j_{L, \mu}\right)
\end{aligned}
$$

where we omit the gauge interactions in $\mathcal{D}_{\mu}$ and axial currents read

$$
J_{L, R}^{\mu} \equiv \sum_{f} \bar{\psi}_{L, R}^{f} \gamma^{\mu} \gamma^{5} \psi_{L, R}^{f}, \quad j_{L}^{\mu} \equiv \sum_{f} \bar{\nu}_{R}^{f c} \gamma^{\mu} \gamma^{5} \nu_{R}^{f c} .
$$

The four-fermion coupling $G$ is unique for all four-fermion operators and high-dimensional fermion operators $(d>6)$ are neglected.

By using the Fierz theorem $[105,106]$, the dimension- 6 four-fermion operators in eq. (2.2) can be written as [107]

$$
\begin{aligned}
& +(G / 2)\left(J_{L}^{\mu} J_{L, \mu}+J_{R}^{\mu} J_{R, \mu}+j_{L}^{\mu} j_{L, \mu}+2 J_{L}^{\mu} j_{L, \mu}\right) \\
& -G \sum_{f f^{\prime}}\left(\bar{\psi}_{L}^{f} \psi_{R}^{f^{\prime}} \bar{\psi}_{R}^{f^{\prime}} \psi_{L}^{f}+\bar{\nu}_{R}^{f c} \psi_{R}^{f^{\prime}} \bar{\psi}_{R}^{f^{\prime}} \nu_{R}^{f c}\right),
\end{aligned}
$$

which preserve the SM gauge symmetries. Equations (2.4) and (2.5) represent repulsive and attractive operators respectively. The former (2.4) are suppressed by the cutoff $\mathcal{O}\left(\Lambda^{-2}\right)$,

\footnotetext{
${ }^{2}$ In the regularized and quantized EC theory [98-100] with a basic space-time cutoff, in addition to dimension-6 four-fermion operators, there are high-dimensional fermion operators $(d>6)$, e.g., $\partial_{\sigma} J^{\mu} \partial^{\sigma} J_{\mu}$, which are suppressed at least by $\mathcal{O}\left(\tilde{a}^{4}\right)$.
} 
and cannot become relevant and renormalizable operators of effective dimension-4. Thus the torsion-free EC theory with the attractive four-fermion operators read,

$$
\begin{aligned}
\mathcal{L}= & \mathcal{L}_{\mathrm{EC}}+\sum_{f} \bar{\psi}_{L, R}^{f} e^{\mu} \mathcal{D}_{\mu} \psi_{L, R}^{f}+\sum_{f} \bar{\nu}_{R}^{f c} e^{\mu} \mathcal{D}_{\mu} \nu_{R}^{f c} \\
& -G \sum_{f f^{\prime}}\left(\bar{\psi}_{L}^{f} \psi_{R}^{f^{\prime}} \bar{\psi}_{R}^{f^{\prime}} \psi_{L}^{f}+\bar{\nu}_{R}^{f c} \psi_{R}^{f^{\prime}} \bar{\psi}_{R}^{f^{\prime}} \nu_{R}^{f c}\right)+\text { h.c. },
\end{aligned}
$$

where the two component Weyl fermions $\psi_{L}^{f}$ and $\psi_{R}^{f}$ respectively are the $S U_{L}(2) \times U_{Y}(1)$ gauged doublets and singlets of the SM. For the sake of compact notations, $\psi_{R}^{f}$ are also used to represent $\nu_{R}^{f}$, which have no any SM quantum numbers. All fermions are massless, they are four-component Dirac fermions $\psi^{f}=\left(\psi_{L}^{f}+\psi_{R}^{f}\right)$, two-component right-handed Weyl neutrinos $\nu_{L}^{f}$ and four-component sterile Majorana neutrinos $\nu_{M}^{f}=\left(\nu_{R}^{f c}+\nu_{R}^{f}\right)$ whose kinetic terms read

$$
\bar{\nu}_{L}^{f} e^{\mu} \mathcal{D}_{\mu} \nu_{L}^{f}, \quad \bar{\nu}_{M}^{f} e^{\mu} \mathcal{D}_{\mu} \nu_{M}^{f}=\bar{\nu}_{R}^{f} e^{\mu} \mathcal{D}_{\mu} \nu_{R}^{f}+\bar{\nu}_{R}^{f c} e^{\mu} \mathcal{D}_{\mu} \nu_{R}^{f c} .
$$

In eq. (2.6), $f$ and $f^{\prime}\left(f, f^{\prime}=1,2,3\right)$ are fermion-family indexes summed over respectively for three lepton families (charge $q=0,-1)$ and three quark families $(q=2 / 3,-1 / 3)$. Eq. (2.6) preserves not only the SM gauge symmetries and global fermion-family symmetries, but also the global symmetries for fermion-numbers conservations. We adopt the effective four-fermion operators (2.6) in the context of a well-defined quantum field theory at the high-energy scale $\Lambda$.

\subsection{SM gauge-symmetric four-fermion operators}

Neglecting the flavor-mixing of three fermion families $\left(f=f^{\prime}\right)$ to simply notations, we explicitly show SM gauge symmetric four-fermion operators in eq. (2.6). In the quark sector, the four-fermion operators are

$$
G\left[\left(\bar{\psi}_{L}^{i a} t_{R a}\right)\left(\bar{t}_{R}^{b} \psi_{\mathrm{Lib}}\right)+\left(\bar{\psi}_{L}^{i a} b_{R a}\right)\left(\bar{b}_{R}^{b} \psi_{\mathrm{Lib}}\right)\right]+\text { "terms", }
$$

where $a, b$ and $i, j$ are the color and flavor indexes of the top and bottom quarks, the quark $S U_{L}(2)$ doublet $\psi_{L}^{i a}=\left(t_{L}^{a}, b_{L}^{a}\right)$ and singlet $\psi_{R}^{a}=t_{R}^{a}, b_{R}^{a}$ are the eigenstates of electroweak interaction. The first and second terms in eq. (2.8) are respectively the four-fermion operators of top-quark channel [81] and bottom-quark channel, whereas "terms" stands for the first and second quark families that can be obtained by substituting $t \rightarrow u, c$ and $b \rightarrow d, s[88,89,109]$.

In the lepton sector with three right-handed sterile neutrinos $\nu_{R}^{\ell}(\ell=e, \mu, \tau)$, the four-fermion operators in terms of gauge eigenstates are,

$$
G\left[\left(\bar{\ell}_{L}^{i} \ell_{R}\right)\left(\bar{\ell}_{R} \ell_{L i}\right)+\left(\bar{\ell}_{L}^{i} \nu_{R}^{\ell}\right)\left(\bar{\nu}_{R}^{\ell} \ell_{L i}\right)+\left(\bar{\nu}_{R}^{\ell c} \nu_{R}^{\ell}\right)\left(\bar{\nu}_{R}^{\ell} \nu_{R}^{\ell c}\right)\right],
$$

preserving all SM gauge symmetries, where the lepton $S U_{L}(2) \operatorname{doublets} \ell_{L}^{i}=\left(\nu_{L}^{\ell}, \ell_{L}\right)$, singlets $\ell_{R}$ and the conjugate fields of sterile neutrinos $\nu_{R}^{\ell c}=i \gamma_{2}\left(\nu_{R}^{\ell}\right)^{*}$. Coming from the second term in eq. (2.6), the last term in eq. (2.9) preserves the symmetry $U_{\text {lepton }}(1)$ for 
the lepton-number conservation, although $\left(\bar{\nu}_{R}^{\ell} \nu_{R}^{\ell c}\right)$ violates the lepton number of family " $\ell$ " by two units.

Similarly, from the second term in eq. (2.6) there are following four-fermion operators

$$
G\left[\left(\bar{\nu}_{R}^{\ell c} \ell_{R}\right)\left(\bar{\ell}_{R} \nu_{R}^{\ell c}\right)+\left(\bar{\nu}_{R}^{\ell c} u_{a, R}^{\ell}\right)\left(\bar{u}_{a, R}^{\ell} \nu_{R}^{\ell c}\right)+\left(\bar{\nu}_{R}^{\ell c} d_{a, R}^{\ell}\right)\left(\bar{d}_{a, R}^{\ell} \nu_{R}^{\ell c}\right)\right],
$$

where quark fields $u_{a, R}^{\ell}=(u, c, t)_{a, R}$ and $d_{a, R}^{\ell}=(d, s, b)_{a, R}$.

\subsection{Four-fermion operators of quark-lepton interactions}

Although the four-fermion operators in eq. (2.6) do not have quark-lepton interactions, we consider the following SM gauge-symmetric four-fermion operators that contain quarklepton interactions [33],

$$
G\left[\left(\bar{\ell}_{L}^{i} e_{R}\right)\left(\bar{d}_{R}^{a} \psi_{L i a}\right)+\left(\bar{\ell}_{L}^{i} \nu_{R}^{e}\right)\left(\bar{u}_{R}^{a} \psi_{L i a}\right)\right]+(\cdots),
$$

where $\ell_{L}^{i}=\left(\nu_{L}^{e}, e_{L}\right)$ and $\psi_{\text {Lia }}=\left(u_{L a}, d_{L a}\right)$ for the first family. The $(\cdots)$ represents for the second and third families with substitutions: $e \rightarrow \mu, \tau, \nu^{e} \rightarrow \nu^{\mu}, \nu^{\tau}$, and $u \rightarrow c, t$ and $d \rightarrow s, b$. The four-fermion operators (2.11) of quark-lepton interactions are not included in eq. (2.6), since leptons and quarks are in separated representations of SM gauge groups. They should be expected in the framework of Einstein-Cartan theory and $\mathrm{SO}(10)$ unification theory $[110,111]$.

In order to study the mass generation of three fermion families by the mixing of three fermion families we generalize the quark-lepton interacting operators (2.11) to

$$
G \sum_{f f^{\prime}}\left\{\left(\bar{\ell}_{L}^{i f} e_{R}^{f^{\prime}}\right)\left(\bar{d}_{R}^{a f^{\prime}} \psi_{L i a}^{f}\right)+\left(\bar{\ell}_{L}^{i f} \nu_{e R}^{f^{\prime}}\right)\left(\bar{u}_{R}^{a f^{\prime}} \psi_{L i a}^{f}\right)\right\},
$$

analogously to the four-fermion operators in eq. (2.6).

\section{Gauge vs mass eigenstates in fermion-family space}

Due to the unique four-fermion coupling $G$ and the global fermion-family $U_{L}(3) \times U_{R}(3)$ symmetry of eq. (2.6), one is allowed to perform chiral transformations $\mathcal{U}_{L} \in U_{L}(3)$ and $\mathcal{U}_{R} \in U_{R}(3)$ so that $f=f^{\prime}$, the four-fermion operators (2.6) are only for each fermion family without the family-flavor-mixing and all fermion fields are Dirac mass eigenstates. In this section, neglecting gauge interactions we discuss the unitary chiral transformations from gauge eigenstates to mass eigenstates in quark and lepton sectors, so as to diagonalize in the fermion-family space the four-fermion operators (2.6) and two-fermion operators $(\psi \bar{\psi})$, the latter is relating to fermion mass matrices.

\subsection{Quark sector}

For the quark sector, the four-fermion operators (2.6) are

$$
-G \sum_{f f^{\prime}}\left[\bar{\psi}_{L}^{f} \psi_{R}^{f^{\prime}} \bar{\psi}_{R}^{f^{\prime}} \psi_{L}^{f}\right]_{2 / 3}-G \sum_{f f^{\prime}}\left[\bar{\psi}_{L}^{f} \psi_{R}^{f^{\prime}} \bar{\psi}_{R}^{f^{\prime}} \psi_{L}^{f}\right]_{-1 / 3},
$$


where the $S U_{L}(2) \times U_{Y}(1)$ doublets $\psi_{L}^{f}$ and singlets $\psi_{R}^{f}$ are the SM gauge eigenstates, $\mathrm{SU}(3)$ color index " $a$ " is summed over $\bar{\psi}_{L}^{a f} \psi_{a R}^{f^{\prime}} \rightarrow \bar{\psi}_{L}^{f} \psi_{R}^{f^{\prime}}, f$ and $f^{\prime}$ are family indexes of three fermion families. The first term is for the $(2 / 3)$-charged sector, $\psi_{R}^{f^{\prime}} \Rightarrow u_{R}^{f^{\prime}}$ represented by the $u$-quark sector $u^{f^{\prime}} \Rightarrow(u, c, t)$, the second term is for the $(-1 / 3)$-charged sector, $\psi_{R}^{f^{\prime}} \Rightarrow d_{R}^{f^{\prime}}$ represented by the $d$-quark sector $d^{f^{\prime}} \Rightarrow(d, s, b)$.

Due to the unique four-fermion coupling $G$ and the global fermion-family $U_{L}^{u}(3) \times$ $U_{R}^{u}(3)$ symmetry for the $u$-quark sector and $U_{L}^{d}(3) \times U_{R}^{d}(3)$ symmetry for the $d$-quark sector in eq. (3.1), we perform four unitary chiral transformations from gauge-eigenstates to mass-eigenstates:

$$
\psi_{L}^{u} \rightarrow \mathcal{U}_{L}^{u} \psi_{L}^{u}, \quad \psi_{R}^{u} \rightarrow \mathcal{U}_{R}^{u} \psi_{R}^{u} ; \quad \mathcal{U}_{L, R}^{u} \in U_{L, R}^{u}(3),
$$

and

$$
\psi_{L}^{d} \rightarrow \mathcal{U}_{L}^{d} \psi_{L}^{d}, \quad \psi_{R}^{d} \rightarrow \mathcal{U}_{R}^{d} \psi_{R}^{d} ; \quad \mathcal{U}_{L, R}^{d} \in U_{L, R}^{d}(3)
$$

so that in eq. (3.1) the fermion-family indexes $f=f^{\prime}$, i.e., $\delta_{f f^{\prime}}$ respectively for the $u$-quark sector and the $d$-quark sector. As a result, all quark fields are mass eigenstates, the fourfermion operators (3.1) are "diagonal" only for each quark family without family-mixing,

$$
-G \sum_{f=1,2,3}\left[\bar{\psi}_{L}^{f} \psi_{R}^{f} \bar{\psi}_{R}^{f} \psi_{L}^{f}\right]_{2 / 3}-G \sum_{f=1,2,3}\left[\bar{\psi}_{L}^{f} \psi_{R}^{f} \bar{\psi}_{R}^{f} \psi_{L}^{f}\right]_{-1 / 3} \cdot
$$

In this representation, the vacuum expectation values of two-fermion operators $\left\langle\psi_{R}^{f} \bar{\psi}_{L}^{f}\right\rangle+$ h.c., i.e., quark-mass matrices are diagonalized in the fermion-family space by the biunitary transformations

$$
\begin{gathered}
M^{u} \Rightarrow M_{\text {diag }}^{u}=\left(m_{1}^{u}, m_{2}^{c}, m_{3}^{t}\right)=\mathcal{U}_{L}^{u \dagger} M^{u} \mathcal{U}_{R}^{u}, \\
M^{d} \Rightarrow M_{\text {diag }}^{d}=\left(m_{1}^{d}, m_{2}^{s}, m_{3}^{b}\right)=\mathcal{U}_{L}^{d \dagger} M^{d} \mathcal{U}_{R}^{d},
\end{gathered}
$$

where all quark masses (eigenvalues) are positive, $\mathcal{U}_{L}$ and $\mathcal{U}_{R}$ are related by

$$
\mathcal{U}_{L}^{u, d}=\mathcal{V}^{u, d} \mathcal{U}_{R}^{u, d}
$$

and $\mathcal{V}^{u, d}$ is an unitary matrix, see for example $[112,113]$.

Using unitary matrices $\mathcal{U}_{L, R}^{u}(3.2)$ and $\mathcal{U}_{L, R}^{d}(3.3)$, up to a diagonal phase matrix we define the unitary quark-family mixing matrices,

$$
\begin{array}{ll}
\mathcal{U}_{L}^{u \dagger} \mathcal{U}_{L}^{d}, & \mathcal{U}_{L}^{u \dagger} \mathcal{U}_{R}^{d}, \\
\mathcal{U}_{R}^{u \dagger} \mathcal{U}_{L}^{d}, & \mathcal{U}_{R}^{u \dagger} \mathcal{U}_{R}^{d}
\end{array}
$$

where the first element is the CKM matrix $U=U_{L}^{q} \equiv \mathcal{U}_{L}^{u \dagger} \mathcal{U}_{L}^{d}$. The experimental values [114] of CKM matrix are adopted to calculate the fermion spectrum in this article. 


\section{$3.2 \quad$ Lepton sector}

For the lepton sector, the four-fermion operators (2.6) are

$$
-G \sum_{f f^{\prime}}\left[\bar{\ell}_{L}^{f} \ell_{R}^{f^{\prime}} \bar{\ell}_{R}^{f^{\prime}} \ell_{L}^{f}+\left(\bar{\ell}_{L}^{f} \nu_{R}^{f^{\prime}}\right)\left(\bar{\nu}_{R}^{f^{\prime}} \ell_{L}^{f}\right)+\left(\bar{\nu}_{R}^{f c} \nu_{R}^{f^{\prime}}\right)\left(\bar{\nu}_{R}^{f^{\prime}} \nu_{R}^{f c}\right)\right]
$$

where Dirac lepton fields $\ell_{L}^{f}$ and $\ell_{R}^{f}$ are the $S M S U_{L}(2)$-doublets and singlets respectively, $\nu_{R}^{f}$ are three sterile (Dirac) neutrinos and $\nu_{R}^{f c}=i \gamma_{2}\left(\nu_{R}^{f}\right)^{*}$ are their the conjugate fields. Analogously to the quark sector (3.1), we perform four unitary chiral transformations from gauge eigenstates to mass eigenstates

$$
\nu_{L} \rightarrow \mathcal{U}_{L}^{\nu} \nu_{L}, \quad \nu_{R} \rightarrow \mathcal{U}_{R}^{\nu} \nu_{R} ; \quad \mathcal{U}_{L, R}^{\nu} \in U_{L, R}^{\nu}(3),
$$

and

$$
\ell_{L} \rightarrow \mathcal{U}_{L}^{\ell} \ell_{L}, \quad \ell_{R} \rightarrow \mathcal{U}_{R}^{\ell} \ell_{R} ; \quad \mathcal{U}_{L, R}^{\ell} \in U_{L, R}^{\ell}(3)
$$

so that in eq. (3.9) the fermion-family indexes $f=f^{\prime}$, i.e., $\delta_{f f^{\prime}}$ respectively for the Dirac $\nu$-neutrino sector $f \mapsto \nu \Rightarrow\left(\nu_{e}, \nu_{\mu}, \nu_{\tau}\right)$ and the charged $\ell$-lepton sector $f \mapsto \ell \Rightarrow(e, \mu, \tau)$. As a result, all lepton fields are mass eigenstates, the four-fermion operators (3.9) are "diagonal" only for each lepton family without family-mixing,

$$
-G \sum_{f=1,2,3}\left[\bar{\ell}_{L}^{f} \ell_{R}^{f} \bar{\ell}_{R}^{f} \ell_{L}^{f}+\left(\bar{\ell}_{L}^{f} \nu_{R}^{f}\right)\left(\bar{\nu}_{R}^{f} \ell_{L}^{f}\right)+\left(\bar{\nu}_{R}^{f c} \nu_{R}^{f}\right)\left(\bar{\nu}_{R}^{f} \nu_{R}^{f c}\right)\right]
$$

and the vacuum expectation values of two-lepton operators $\left\langle\ell_{R}^{f} \bar{\ell}_{L}^{f}\right\rangle+$ h.c., $\left\langle\nu_{L}^{f} \bar{\nu}_{R}^{f}\right\rangle+$ h.c. and $\left\langle\nu_{R}^{f c} \bar{\nu}_{R}^{f}\right\rangle+$ h.c., i.e., lepton-mass matrices are diagonalized in the fermion-family space by the biunitary transformations

$$
M^{\ell} \Rightarrow M_{\text {diag }}^{\ell}=\left(m_{1}^{e}, m_{2}^{\mu}, m_{3}^{\tau}\right)=\mathcal{U}_{L}^{\ell \dagger} M^{\ell} \mathcal{U}_{R}^{\ell}
$$

and

$$
\begin{aligned}
M^{\nu} \Rightarrow M_{\text {diag }}^{\nu} & =\left(m_{1}^{\nu_{e}}, m_{2}^{\nu_{\mu}}, m_{3}^{\nu_{\tau}}\right)=\mathcal{U}_{L}^{\nu \dagger} M^{\nu} \mathcal{U}_{R}^{\nu} \\
M \Rightarrow M_{\text {diag }} & =\left(m_{1}^{M}, m_{2}^{M}, m_{3}^{M}\right)=\left(\mathcal{U}_{R}^{\nu \dagger}\right)^{*} M \mathcal{U}_{R}^{\nu}=\tilde{\mathcal{U}}_{R}^{\nu} M \mathcal{U}_{R}^{\nu}
\end{aligned}
$$

where all lepton masses (eigenvalues) are positive. The Dirac neutrino mass matrix can be expressed as $M^{\nu}=\mathcal{H}^{\nu} \mathcal{V}^{\nu}$, and $\mathcal{H}^{\nu}$ is a hermitian matrix. The $\mathcal{U}_{L}^{\nu}$ and $\mathcal{U}_{R}^{\nu}$ are related by

$$
\mathcal{U}_{L}^{\nu}=\mathcal{V}^{\nu} \mathcal{U}_{R}^{\nu}
$$

and $\mathcal{V}^{\nu}$ is an unitary matrix. This also applies for charged lepton sector $(\nu \rightarrow \ell)$, see $[112,113]$. In the following sections, we adopt the bases of mass-eigenstates and drop the subscriptions 1,2,3 for simplifying the notations in $M_{\text {diag }}^{u, d}(3.5),(3.6), M_{\text {diag }}^{\nu, \ell}(3.14),(3.13)$ and $M_{\text {diag }}(3.15)$. 
Using unitary matrices $\mathcal{U}_{L, R}^{\nu}(3.10)$ and $\mathcal{U}_{L, R}^{\ell}(3.11)$, up to a phase we define the unitary lepton-family mixing matrices,

$$
\begin{array}{ll}
\mathcal{U}_{L}^{\nu \dagger} \mathcal{U}_{L}^{\ell}, & \mathcal{U}_{L}^{\nu \dagger} \mathcal{U}_{R}^{\ell}, \\
\mathcal{U}_{R}^{\nu^{\dagger}} \mathcal{U}_{L}^{\ell}, & \mathcal{U}_{R}^{\nu \dagger} \mathcal{U}_{R}^{\ell}
\end{array}
$$

where the first element is the PMNS matrix $U^{\ell}=U_{L}^{\ell} \equiv \mathcal{U}_{L}^{\nu^{\dagger}} \mathcal{U}_{L}^{\ell}$. We adopt the most recent updated range [115] of PMNS matrix elements to calculate the fermion spectrum in this article. We can also define the notation for the last element

$$
U_{R}^{\ell} \equiv \mathcal{U}_{R}^{\nu \dagger} \mathcal{U}_{R}^{\ell}
$$

that will be used later. Note that each of the unitary matrices $\mathcal{U}_{L}^{\nu, \ell, u, d}$ in eqs. (3.2), (3.3) and (3.10), (3.11) is unique up to a diagonal phase matrix $P_{\text {diag }}^{\nu, \ell, d}=\left(e^{i \phi_{1}}, e^{i \phi_{2}}, e^{i \phi_{3}}\right)$ [112]. These phase degrees of freedom are used here to ensure all mass eigenvalues are positive, and we do not consider the question of CP-violation at the moment.

The Majorana mass matrix $M(3.15)$ is a symmetric matrix, relating to the vacuum expectation value of two fermion operator $\left(\bar{\nu}_{R}^{f c} \nu_{R}^{f}\right)$. Using (3.16), eq. (3.15) can be rewritted as,

$$
M_{\text {diag }}=\left(\mathcal{U}_{L}^{\nu \dagger} \mathcal{V}^{\nu}\right)^{*} M \mathcal{U}_{R}^{\nu}=\left(\mathcal{U}_{L}^{\nu \dagger} \mathcal{V}^{\nu} M \mathcal{U}_{R}^{\nu}\right)^{*}=\mathcal{U}_{L}^{\nu \dagger}\left(\mathcal{V}^{\nu} M\right) \mathcal{U}_{R}^{\nu},
$$

where in the last equality we assume the CP-conservation for Majorana fields $\nu_{R}^{f c}$ and $\nu_{R}^{f}$ so that their matrix $M=M^{*}$ and transformation $\left(\mathcal{U}_{R}^{\nu}\right)^{*}=\mathcal{U}_{R}^{\nu}$ are real. Comparing eq. (3.14) to eq. (3.19), we find that the Dirac neutrino mass matrix $M^{\nu}=H^{\nu} \mathcal{V}^{\nu}$ (3.14) and the matrix $\mathcal{V}^{\nu} M$ (3.19) are diagonalized by the same biunitary transformation, and they have common eigen-vectors. In fact, both mass matrices are related to the $\bar{\nu}_{R}^{f}$-field condensation, i.e., the Dirac mass matrix $M^{\nu} \sim\left\langle\nu_{L}^{f} \bar{\nu}_{R}^{f}\right\rangle$ and the Majorana mass matrix $M \sim\left\langle\nu_{R}^{f c} \bar{\nu}_{R}^{f}\right\rangle$. Therefore, we expect that they should have a similar structure of eigenvalues, for example the normal hierarchy structure, $m_{1}^{\nu_{e}}<m_{2}^{\nu_{\mu}}<m_{3}^{\nu_{\tau}}$ in eq. (3.14) and $m_{1}^{M}<m_{2}^{M}<m_{3}^{M}$ in eq. (3.15). We will present detail discussions on the first, second and third four-fermion operators involving $\nu_{R}^{f}$ in eq. (3.12), as well as the Dirac mass matrix $M^{\nu}(3.14)$, the Majorana mass matrix $M(3.15)$ and mixing matrix (3.17) in the last section specially for neutrinos.

\subsection{Quark-lepton interaction sector}

Using the same chiral transformations (3.2), (3.3), (3.10) and (3.11) in quark and lepton sectors, we obtain that in the fermion-family space the four-fermion operators (2.12) are "diagonal" $\left(f=f^{\prime}\right)$, and we rewrite these operators in terms of Dirac mass eigenstates

$$
\begin{gathered}
\text { Eq. (2.12)=G } \sum_{f f^{\prime}}\left\{\left[\left(\bar{\ell}_{L}^{i} \mathcal{U}_{L}^{e \dagger}\right)^{f}\left(\mathcal{U}_{R}^{e} e_{R}\right)^{f^{\prime}}\right]\left[\left(\bar{d}_{R}^{a} \mathcal{U}_{R}^{d \dagger}\right)^{f^{\prime}}\left(\mathcal{U}_{L}^{d} \psi_{\text {Lia }}\right)^{f}\right]\right. \\
\left.+\left[\left(\bar{\ell}_{L}^{i} \mathcal{U}_{L}^{\nu \dagger}\right)^{f}\left(\mathcal{U}_{R}^{\nu} \nu_{e R}\right)^{f^{\prime}}\right]\left[\left(\bar{u}_{R}^{a} \mathcal{U}_{R}^{u \dagger}\right)^{f^{\prime}}\left(\mathcal{U}_{L}^{u} \psi_{L i a}\right)^{f}\right]\right\} \\
=G \sum_{f f^{\prime}}\left\{\left[\begin{array}{c}
{\left[\bar{\ell}_{L}^{i f}\left(U_{R}^{d e} e_{R}\right)^{f^{\prime}}\right]\left[\bar{d}_{R}^{a f^{\prime}}\left(U_{L}^{e d} \psi_{\text {Lia }}\right)^{f}\right]} \\
\left.+\left[\bar{\ell}_{L}^{i f}\left(U_{R}^{u \nu} \nu_{e R}\right)^{f^{\prime}}\right]\left[\bar{u}_{R}^{a f^{\prime}}\left(U_{L}^{\nu u} \psi_{L i a}\right)^{f}\right]\right\} .
\end{array}\right.\right.
\end{gathered}
$$


where $f=f^{\prime}$ and four unitary mixing matrices between lepton and quark families are defined by

$$
\begin{aligned}
& U_{R}^{d e}=\mathcal{U}_{R}^{d \dagger} \mathcal{U}_{R}^{e}, \quad U_{L}^{e d}=\mathcal{U}_{L}^{e \dagger} \mathcal{U}_{L}^{d}, \\
& U_{R}^{u \nu}=\mathcal{U}_{R}^{u \dagger} \mathcal{U}_{R}^{\nu}, \quad U_{L}^{\nu u}=\mathcal{U}_{L}^{\nu \dagger} \mathcal{U}_{L}^{u},
\end{aligned}
$$

analogously to the mixing matrices (3.8) in the quark sector and (3.17) in the lepton sector. Relating to the $U_{L}^{d}\left(U_{L}^{u}\right)$ in the CKM matrix $U_{L}^{u \dagger} U_{L}^{d}$, the matrix $U_{L}^{e d}\left(U_{L}^{\nu u}\right)$ is expected to have a hierarchy structure, namely, in the fermion-family space the diagonal elements are the order of unit, while the off-diagonal elements are much smaller than the order of unit.

Equations (3.8), (3.17), and (3.22) give the mixing matrices of mass and gauge eigenstates of three fermion families, due to the $W^{ \pm}$-boson interaction and four-fermion interactions (2.2). The elements of these unitary matrices are not completely independent each other, as we have already known from the CKM and PMNS matrices. As will be shown, these mixing matrices and mass spectra of the SM fermions are fundamental, and closely related.

Henceforth, all fermion fields are mass eigenstates, two-fermion mass operators and four-fermion operators are "diagonal" in the fermion-family space.

\section{Spontaneous symmetry breaking}

In this section, we briefly recall and discuss that in the IR-domain of the IR-stable fixed point $G_{c}$, the relevant four-fermion operator (2.8) undergoes the SSB and becomes an effectively bilinear and renormalizable Lagrangian that follows the RG-equations to approach the SM physics in the low-energy. This is necessary and fundamental for studying the origin of SM fermion masses in this article.

\subsection{The IR fixed-point domain and only top-quark mass generated via the SSB}

Apart from what is possible new physics at the scale $\Lambda$ explaining the origin of these effective four-fermion operators (2.6), it is essential and necessary to study: (i) which dynamics of these operators undergo in terms of their couplings as functions of running energy scale $\mu$; (ii) associating to these dynamics where the infrared (IR) or ultraviolet (UV) stable fixed point of physical couplings locates; (iii) in the domains (scaling regions) of these stable fixed points, which physically relevant operators that become effectively dimensional-4 renormalizable operators following RG equations (scaling laws), while other irrelevant operators are suppressed by the cutoff at least $\mathcal{O}\left(\Lambda^{-2}\right)$.

In the IR-domain of the IR-stable fixed point $G_{c}$, the four-fermion operator (1.1) was shown [81] to become physically relevant and renormalizable operators of effective dimension-4, due to the SSB dynamics of NJL-type. Namely, the Lagrangian (1.1) becomes the effective SM Lagrangian with bilinear top-quark mass term and Yukawa-coupling to the composite Higgs boson $H$, which obeys the RG-equations approaching to the low-energy SM physics characterized by the energy scale $v \approx 239.5 \mathrm{GeV}$. In addition, the top-quark 
and composite Higgs-boson masses are correctly obtained by solving RG-equations with the appropriate non-vanishing form-factor of the Higgs boson in TeV scales [88, 89].

It seems that via the SSB dynamics the four-fermion operator the quark-condensation $M_{f f^{\prime}}^{q}=-G\left\langle\bar{\psi}^{f} \psi^{f^{\prime}}\right\rangle / 2 N_{c}=m \delta_{f f^{\prime}} \neq 0$ (the color number $N_{c}$ ), and two diagonal mass matrices $M_{\text {diag }}^{u}=\left(m_{1}^{u}, m_{2}^{c}, m_{3}^{t}\right)$ and $M_{\text {diag }}^{d}=\left(m_{1}^{d}, m_{2}^{s}, m_{3}^{b}\right)$ of quark sectors $q=2 / 3$ and $q=$ $-1 / 3$ satisfying $3+3$ mass-gap equations of NJL type. It was demonstrated [109] that as an energetically favorable solution of the SSB ground state of the SM, only top quark is massive $\left(m_{t}^{\mathrm{sb}}=-G\left\langle\bar{\psi}_{t} \psi_{t}\right\rangle \neq 0\right)$, otherwise there would be more Goldstone modes in addition to those become the longitudinal modes of massive gauge bosons $W^{ \pm}$and $Z^{0}$. Extra Goldstone modes have positive contributions to the ground-state energy, and thus make the ground-state energy increase. As a result, $M_{\text {diag }}^{u}=\left(0,0, m_{3}^{t}\right)$ and $M_{\text {diag }}^{d}=(0,0,0)$, only the top-quark channel (1.1) undergoes the SSB dynamics and becomes relevant operator following the RG equations in the IR domain.

We turn to the lepton sector. The first and second four-fermion operators in eq. (2.9) or (3.12) relate to the lepton Dirac mass matrix. At first glance, it seems that the four-lepton operators undergo the SSB leading to the lepton-condensation $M_{f f^{\prime}}^{\ell}=$ $-G\left\langle\bar{\ell}^{f} \ell^{f^{\prime}}\right\rangle / 2=m_{\ell} \delta_{f f^{\prime}}$, and two diagonal mass matrices $M_{\text {diag }}^{\nu}=\left(m_{1}^{\nu_{e}}, m_{2}^{\nu_{\mu}}, m_{3}^{\nu_{\tau}}\right)$ and $M_{\mathrm{diag}}^{\ell}=\left(m_{1}^{e}, m_{2}^{\mu}, m_{3}^{\tau}\right)$ of the lepton sector $(q=0$ and $q=-1)$ satisfying $3+3$ mass-gap equations of NJL type. Actually, the first and second four-fermion operators in eq. (2.9) or (3.12) do not undergo the SSB and two lepton Dirac mass matrices $(q=0$ and $q=-1)$ are zero matrices, i.e., $M_{\text {diag }}^{\nu}=(0,0,0)$ and $M_{\text {diag }}^{\ell}=(0,0,0)$. The reason is that the effective four-lepton coupling $\left(G N_{c}\right) / N_{c}$ is $N_{c}$-smaller than the critical value $\left(G N_{c}\right)$ of the effective four-quark coupling for the SSB in the quark sector, in addition to the reason of energetically favorable solution for the SSB ground state discussed above.

Therefore, in the IR-domain where the SSB occurs, except the top quark, all quarks and leptons are massless and their four-fermion operators (3.4) and (3.12), as well as repulsive four-fermion operators (2.4), are irrelevant dimension-6 operators. Their treelevel amplitudes of four-fermion scatterings are suppressed $\mathcal{O}\left(\Lambda^{-2}\right)$, thus such deviations from the SM are experimentally inaccessible today [107].

The heaviest quark which acquires its mass via the SSB is identified and named as the top quark. The heaviest fermion family is named as the third fermion family of fermions $\nu_{\tau}, \tau, t, b$, where the top quark is. We study their mass spectra in ref. [97]. As will be discussed, these third-family quarks and leptons are grouped together for their heavy masses, due to the fermions $\nu_{\tau}, \tau, b$ have the largest mixing with the top quark.

\subsection{The $\langle\bar{t} t\rangle$-condensate model}

We briefly recall the BHL $\langle\bar{t} t\rangle$-condensate model [81] for the full effective Lagrangian of the low-energy SM in the IR-domain, and the analysis [88, 89] of RG equations based on experimental boundary conditions, as well as experimental indications of the composite Higgs boson. 


\subsubsection{The scaling region of the IR-stable fixed point}

Using the approach of large $N_{c}$-expansion with a fixed value $G N_{c}$, it is shown [81] that the top-quark channel of operators (2.8) undergoes the SSB dynamics in the IR-domain of IR-stable fixed point $G_{c}$, leading to the generation of top-quark mass

$$
m_{t}=-\left(1 / 2 N_{c}\right) G \sum_{a}\left\langle\bar{t}^{a} t_{a}\right\rangle=-\left(G / N_{c}\right) \sum_{a}\left\langle\bar{t}_{L}^{a} t_{a R}\right\rangle
$$

by the $\langle\bar{t} t\rangle$-condensate. As a result, the $\Lambda^{2}$-divergence (tadpole-diagram) is removed by the mass gap-equation, the top-quark channel of four-fermion operator (1.1) becomes physically relevant and renormalizable operators of effective dimension-4. Namely, the effective SM Lagrangian with the bilinear top-quark mass term and Yukawa coupling to the composite Higgs boson $H$ at the low-energy scale $\mu$ is given by [81]

$$
\begin{aligned}
L= & L_{\text {kinetic }}+g_{t 0}\left(\bar{\Psi}_{L} t_{R} H+\text { h.c. }\right)+\Delta L_{\text {gauge }} \\
& +Z_{H}\left|D_{\mu} H\right|^{2}-m_{H}^{2} H^{\dagger} H-\frac{\lambda_{0}}{2}\left(H^{\dagger} H\right)^{2}
\end{aligned}
$$

all renormalized quantities received fermion-loop contributions are defined with respect to the low-energy scale $\mu$. The conventional renormalization $Z_{\psi}=1$ for fundamental fermions and the unconventional wave-function renormalization (form factor) $\tilde{Z}_{H}$ for the composite Higgs boson are adopted

$$
\tilde{Z}_{H}(\mu)=\frac{1}{\bar{g}_{t}^{2}(\mu)}, \bar{g}_{t}(\mu)=\frac{Z_{H Y}}{Z_{H}^{1 / 2}} g_{t 0} ; \quad \tilde{\lambda}(\mu)=\frac{\bar{\lambda}(\mu)}{\bar{g}_{t}^{4}(\mu)}, \bar{\lambda}(\mu)=\frac{Z_{4 H}}{Z_{H}^{2}} \lambda_{0},
$$

where $Z_{H Y}$ and $Z_{4 H}$ are proper renormalization constants of the Yukawa coupling and quartic coupling in eq. (4.2). The SSB-generated top-quark mass $m_{t}(\mu)=\bar{g}_{t}^{2}(\mu) v / \sqrt{2}$. The composite Higgs-boson is described by its pole-mass $m_{H}^{2}(\mu)=2 \tilde{\lambda}(\mu) v^{2}$, form-factor $\tilde{Z}_{H}(\mu)=1 / \bar{g}_{t}^{2}(\mu)$, and effective quartic coupling $\tilde{\lambda}(\mu)$, provided that $\tilde{Z}_{H}(\mu)>0$ and $\tilde{\lambda}(\mu)>0$ are obeyed. After the proper wave-function renormalization $\tilde{Z}_{H}(\mu)$, the Higgs boson behaves as an elementary particle, as long as $\tilde{Z}_{H}(\mu) \neq 0$ is finite.

In the IR-domain where the SM of particle physics is realized, the full one-loop RG equations for running couplings $\bar{g}_{t}\left(\mu^{2}\right)$ and $\bar{\lambda}\left(\mu^{2}\right)$ read

$$
\begin{aligned}
16 \pi^{2} \frac{d \bar{g}_{t}}{d t} & =\left(\frac{9}{2} \bar{g}_{t}^{2}-8 \bar{g}_{3}^{2}-\frac{9}{4} \bar{g}_{2}^{2}-\frac{17}{12} \bar{g}_{1}^{2}\right) \bar{g}_{t}, \\
16 \pi^{2} \frac{d \bar{\lambda}}{d t} & =12\left[\bar{\lambda}^{2}+\left(\bar{g}_{t}^{2}-A\right) \bar{\lambda}+B-\bar{g}_{t}^{4}\right], \quad t=\ln \mu
\end{aligned}
$$

where one can find $A, B$ and RG equations for running gauge couplings $\bar{g}_{1,2,3}^{2}$ in eqs. (4.7), (4.8) of ref. [81]. The solutions to these ordinary differential equations are uniquely determined, once the boundary conditions are fixed.

In ref. [88, 89], we analyzed the RG equations (4.4) and (4.5) by using the boundary conditions based on the experimental values of top-quark and Higgs-boson masses, $m_{t} \approx$ $173 \mathrm{GeV}$ and $m_{H} \approx 126 \mathrm{GeV}$, i.e., the mass-shell conditions

$$
m_{t}\left(m_{t}\right)=\bar{g}_{t}^{2}\left(m_{t}\right) v / \sqrt{2} \approx 173 \mathrm{GeV}, \quad m_{H}\left(m_{H}\right)=\left[2 \tilde{\lambda}\left(m_{H}\right)\right]^{1 / 2} v \approx 126 \mathrm{GeV}
$$



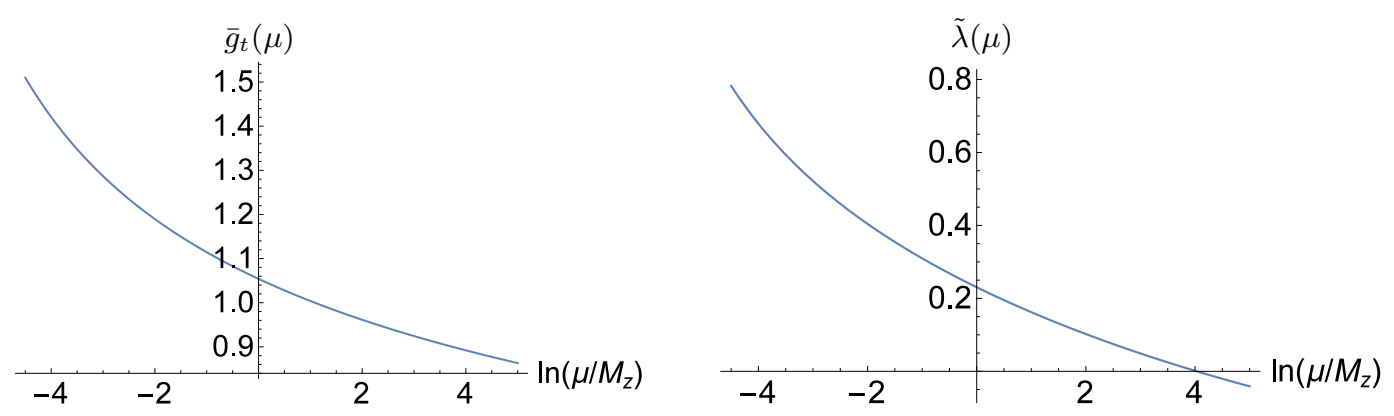

Figure 1. Using experimentally measured SM quantities (including $m_{t}$ and $m_{H}$ ) as boundary values, we uniquely solve the RG equations for the composite Higgs-boson model [81], we find [88, 89] the effective top-quark Yukawa coupling $\bar{g}_{t}(\mu)$ (left) and effective Higgs quartic coupling $\tilde{\lambda}(\mu)$ (right) in the range $1.0 \mathrm{GeV} \lesssim \mu \lesssim 13.5 \mathrm{TeV}$. Note that $\tilde{\lambda}(\mathcal{E})=0$ at $\mathcal{E} \approx 5.14 \mathrm{TeV}$ and $\tilde{\lambda}(\mu)<0$ for $\mu>\mathcal{E}$.

to determine the solutions for $\tilde{Z}_{H}(\mu)$ and $\tilde{\lambda}(\mu)$ in the IR-domain of the energy scale $v=$ $239.5 \mathrm{GeV}$. As a result, we obtained the unique solution (see figure 1) for the composite Higgs-boson model (1.1) or (4.2) as well as at the energy scale $\mathcal{E}$

$$
\mathcal{E} \approx 5.1 \mathrm{TeV}, \quad \tilde{Z}_{H} \approx 1.26, \quad \tilde{\lambda}(\mathcal{E})=0 .
$$

More detailed discussions can be found in ref. [97]. The interested readers are referred to ref. [88] for the resolution to drastically fine-tuning problem.

\subsubsection{Experimental indications of composite Higgs boson?}

To end this section, we discuss the experimental indications of composite Higgs boson. In the IR-domain, the dynamical symmetry breaking of four-fermion operator $G\left(\bar{\psi}_{L}^{i a} t_{R a}\right)\left(\bar{t}_{R}^{b} \psi_{\mathrm{Lib}}\right)$ of the top-quark channel (2.8) accounts for the masses of top quark, $W$ and $Z$ bosons as well as a Higgs boson composed by a top-quark pair ( $\bar{t} t)$ [81]. It is shown $[88,89]$ that this mechanism consistently gives rise to the top-quark and Higgs masses, provided the appropriate value of non-vanishing form-factor of composite Higgs boson at the high-energy scale $\mathcal{E} \gtrsim 5 \mathrm{TeV}$.

Due to its finite form factor (4.7), the composite Higgs boson behaves as if an elementary Higgs particle, the deviation from the SM is too small to be identified by the low-energy collider signatures at the present level [89]. More detailed analysis of the composite Higgs boson phenomenology is indeed needed. It deserves another lengthy article for this issue, nevertheless we present a brief discussion on this aspect. The non-vanishing form-factor $\tilde{Z}_{H}(\mu)$ means that after conventional wave-function and vertex renormalizations $Z_{H}^{1 / 2} H \rightarrow H, Z_{H Y} g_{t 0} \rightarrow g_{t 0}$ and $Z_{4 H} \lambda_{0} \rightarrow \lambda_{0}$ [see eqs. (4.2) and (4.3)], the composite Higgs boson behaves as an elementary particle. The non-vanishing form-factor of composite Higgs boson is in fact related to the effective Yukawa-coupling of Higgs boson and top quark, i.e., $\tilde{Z}_{H}^{-1 / 2}(\mu)=\bar{g}_{t}(\mu)$ of eq. (4.2). The effective Yukawa coupling $\bar{g}_{t}(\mu)$ and quartic coupling $\tilde{\lambda}(\mu)$ monotonically decrease with the energy scale $\mu$ increasing in the range $m_{H}<\mu<\mathcal{E} \approx 5 \mathrm{TeV}$ (see figure 3 ). This means that the composite Higgs boson becomes more tightly bound as the the energy scale $\mu$ increases. 
On the other hand, that the effective Yukawa coupling $\bar{g}_{t}(\mu)$ and quartic coupling $\tilde{\lambda}(\mu)$ decrease as the energy scale $\mu$ increases in the range $m_{H}<\mu<\mathcal{E}$ implies some effects on the rates or cross-sections of the following three dominate processes of Higgs-boson production and decay $[8,108]$ or other relevant processes. Two-gluon fusion produces a Higgs boson via a top-quark loop, which is proportional to the effective Yukawa coupling $\bar{g}_{t}(\mu)$. Then, the produced Higgs boson decays into the two-photon state by coupling to a top-quark loop, and into the four-lepton state by coupling to two massive $W$-bosons or two massive $Z$-bosons. Due to the $\bar{t} t$-composite nature of Higgs boson, the one-particle-irreducible (1PI) vertexes of Higgs-boson coupling to a top-quark loop, two massive $W$-bosons or two massive $Z$-bosons are proportional to the effective Yukawa coupling $\bar{g}_{t}(\mu)$. As a result, both the Higgs-boson decaying rate to each of these three channels and total decay rate are proportional to $\bar{g}_{t}^{2}(\mu)$, which does not affect on the branching ratio of each Higgsdecay channel. The energy scale $\mu$ is actually the Higgs-boson energy, representing the total energy of final states, e.g., two-photon state and four-lepton states, into which the produced Higgs boson decays.

These discussions imply that the resonant amplitude (number of events) of two-photon invariant mass $m_{\gamma \gamma} \approx 126 \mathrm{GeV}$ and/or four-lepton invariant mass $m_{4 l} \approx 126 \mathrm{GeV}$ is expected to become smaller as the produced Higgs-boson energy $\mu$ increases, i.e., the energy of final two-photon and/or four-lepton states increases, when the CM energy $\sqrt{s}$ of LHC $p p$ collisions increases with a given luminosity. Suppose that the total decay rate or each channel decay rate of the SM Higgs boson is measured at the Higgs-boson energy $\mu=m_{t}$ and the SM value of Yukawa coupling $\bar{g}_{t}^{2}\left(m_{t}\right)=2 m_{t}^{2} / v \approx 1.04$ (see figure 3 ). In this scenario of composite Higgs boson, as the Higgs-boson energy $\mu$ increases to $\mu=2 m_{t}$, the Yukawa coupling $\bar{g}_{t}^{2}\left(2 m_{t}\right) \approx 0.98$ (see figure 3 ), the variation of total decay rate or each channel decay rate is expected to be $6 \%$ for $\Delta \bar{g}_{t}^{2} \approx 0.06$. Analogously, the variation is expected to be $9 \%$ at $\mu=3 m_{t}, \bar{g}_{t}^{2}\left(3 m_{t}\right) \approx 0.95$ or $11 \%$ at $\mu=4 m_{t}, \bar{g}_{t}^{2}\left(4 m_{t}\right) \approx 0.93$ (see figure 3). These variations are still too small to be clearly distinguished by the present LHC experiments. Nevertheless, these effects are the nonresonant new signatures of lowenergy collider that show the deviations of this scenario from the SM. We see that the induced (1PI) Yukawa couplings $\bar{g}_{b}(\mu)$ and $\bar{g}_{\tau}(\mu)$ [97], as well as $\bar{g}_{f}(\mu)$ (the present article) of composite Higgs boson to the bottom-quark, tau-lepton and other fermions also weakly decrease with increasing Higgs-boson energy, this implies a slight decrease of number of dilepton events in the Drell-Yan process.

\section{$5 \quad$ Origins of explicit symmetry breaking}

We study in this section, once the top quark mass is generated by the SSB at the scale $\mathcal{E}$, other quarks and leptons acquire their masses by the explicit symmetry breaking (ESB), via both quark-lepton interactions (2.12) and fermion-family mixing. We henceforth indicate the SSB-generated top-quark mass $m_{t}^{\mathrm{sb}}$ and ESB-generated masses $m_{f}^{\mathrm{eb}}$ of other fermions, they represent bare masses at the cutoff energy scale $\mathcal{E}$ of the symmetry breaking phase.

\subsection{Quark-lepton interactions}

Once quarks acquire their bare masses $m_{u}^{\mathrm{eb}}$ and $m_{d}^{\mathrm{eb}}$, due to the ESB or the SSB for top quark only at the scale $\mathcal{E}$, four-fermion operators (3.21) contribute, via the tadpole 

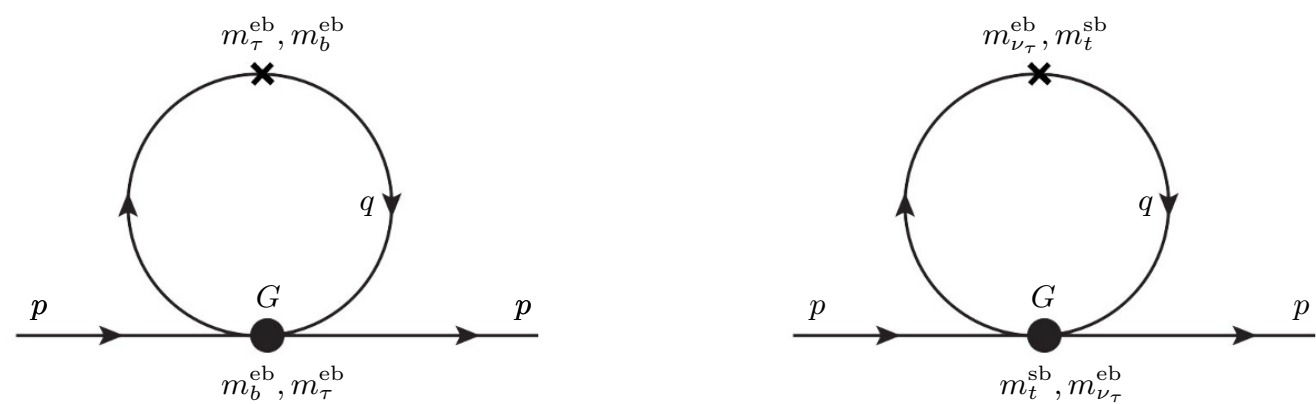

Figure 2. Using the third fermion family as an example, we show the tadpole diagrams of quarklepton interactions (2.11) that contribute quark and lepton ESB masses $m^{\mathrm{eb}}$ to mass-gap equations or SD-equations (6.4)-(6.11). The mixing matrix element $U_{L}^{\tau b} U_{R}^{b \tau}$ or $U_{L}^{\tau b \dagger} U_{R}^{b \tau \dagger}$ associates to the interacting vertex $G$ in the left diagram. The mixing matrix element $U_{L}^{\nu_{\tau} t} U_{R}^{t \nu_{\tau}}$ or $U_{L}^{\nu_{\tau} t \dagger} U_{R}^{t \nu_{\tau} \dagger}$ associates to the interacting vertex $G$ in the right diagram. The mixing matrix elements with the first and second fermion family are neglected.

diagram in figure 2, the bare mass terms $m_{\ell_{\nu}}^{\mathrm{eb}}$ and $m_{\ell}^{\mathrm{eb}}$ in mass-gap equations in the lepton sector. Vice versa once leptons acquire their bare masses, via the same tadpole diagram in figure 2, four-fermion operators (3.21) contribute the bare mass terms $m_{u}^{\mathrm{eb}}$ and $m_{d}^{\mathrm{eb}}$ in mass-gap equations in the quark sector. The superscript "sb" indicates the mass generated by the SSB. The superscript "eb" indicates the mass generated by the ESB. These are bare fermion masses at the energy scale $\mathcal{E}$. As a result, from eq. (3.21) we obtain the relationships between quark and lepton diagonal mass matrices,

$$
\left[m_{e, \mu, \tau}^{\mathrm{eb}}\right]=\left(1 / N_{c}\right) U_{L}^{e d}\left[m_{d, s, b}^{\mathrm{eb}}\right] U_{R}^{d e}, \quad\left[m_{\nu_{e}, \nu_{\mu}, \nu_{\tau}}^{\mathrm{eb}}\right]=\left(1 / N_{c}\right) U_{L}^{\nu u}\left[m_{u, c, t}^{\mathrm{eb}}\right] U_{R}^{u \nu}
$$

where the four diagonal matrices are

$$
\begin{aligned}
{\left[m_{e, \mu, \tau}^{\mathrm{eb}}\right] } & \equiv \operatorname{diag}\left(m_{e}^{\mathrm{eb}}, m_{\mu}^{\mathrm{eb}}, m_{\tau}^{\mathrm{eb}}\right), & {\left[m_{\nu_{e}, \nu_{\mu}, \nu_{\tau}}^{\mathrm{eb}}\right] } & \equiv \operatorname{diag}\left(m_{\nu_{e}}^{\mathrm{eb}}, m_{\nu_{\mu}}^{\mathrm{eb}}, m_{\nu_{\tau}}^{\mathrm{eb}}\right), \\
{\left[m_{d, s, b}^{\mathrm{eb}}\right] } & \equiv \operatorname{diag}\left(m_{d}^{\mathrm{eb}}, m_{s}^{\mathrm{eb}}, m_{b}^{\mathrm{eb}}\right), & {\left[m_{u, c, t}^{\mathrm{eb}}\right] } & \equiv \operatorname{diag}\left(m_{u}^{\mathrm{eb}}, m_{c}^{\mathrm{eb}}, m_{t}^{\mathrm{sb}}\right),
\end{aligned}
$$

and their corresponding non-diagonal mass matrices are eqs. (3.5), (3.6), (3.14) and (3.13). The unitary quark-lepton mixing matrices (3.22) make the transformations from lepton diagonal mass-matrices to quark diagonal mass-matrices, vice versa.

Apart from the SSB-generated top-quark mass $m_{t}^{\mathrm{sb}}$, all other fermion masses $m_{f}^{\mathrm{eb}}$ are ESB-generated and related to the top-quark mass $m_{t}^{\mathrm{sb}}$ by the mixing matrices (3.21) or (3.22). Analogously to eq. (4.1) for the $\langle\bar{t} t\rangle$, in terms of two-fermion operators in mass eigenstates, we define Dirac quark, lepton and neutrino bare masses at the energy scale $\mathcal{E}$, as well as Majorana mass $M$

$$
\begin{aligned}
& m_{\mathrm{q}}^{\mathrm{eb}}=-\left(1 / 2 N_{c}\right) G \sum_{a}\left\langle\bar{\psi}^{a} \psi_{a}\right\rangle=-G / N_{c} \sum_{a}\left\langle\bar{\psi}_{L}^{a} \psi_{a R}\right\rangle, \\
& m_{\ell}^{\mathrm{eb}}=-(1 / 2) G\langle\bar{\ell} \ell\rangle=-G\left\langle\bar{\ell}_{L} \ell_{R}\right\rangle, \\
& m_{\nu^{\ell}}^{\mathrm{eb}}=-(1 / 2) G\left\langle\bar{\nu}^{\ell} \nu^{\ell}\right\rangle=-G\left\langle\bar{\nu}_{L}^{\ell} \nu_{R}^{\ell}\right\rangle, \\
& m_{\nu}^{\mathrm{M}}=-G \sum_{\ell}\left\langle\bar{\nu}_{R}^{\mathrm{c} \ell} \nu_{R}^{\ell}\right\rangle
\end{aligned}
$$


where the color index $a$ is summed over in eq. (5.4) and the lepton-family index $\ell$ is summed over in eq. (5.7), whereas in eqs. (5.5) and (5.6) $\ell=e, \mu, \tau$ respectively indicates each of three fermion families (mass eigenstates). In eqs. (5.4)-(5.7), the notation $\langle\cdots\rangle$ does not represent new SSB-condensates, but the 1PI functions of fermion mass operator $\bar{\psi}_{L} \psi_{R}$, i.e., the self-energy functions $\Sigma_{f}$ that satisfy the self-consistent SD equations or mass-gap equations.

We use the quark-lepton interaction of the third family as an example to show the quark-lepton interactions contribute to the SD-equations of fermion self-energy functions [97]. The quark-lepton interaction (2.11) of the third family reads

$$
G\left[\left(\bar{\ell}_{L}^{i} \tau_{R}\right)\left(\bar{b}_{R}^{a} \psi_{\text {Lia }}\right)+\left(\bar{\ell}_{L}^{i} \nu_{R}^{\tau}\right)\left(\bar{t}_{R}^{a} \psi_{\text {Lia }}\right)\right],
$$

where $\ell_{L}^{i}=\left(\nu_{L}^{\tau}, \tau_{L}\right)$ and $\psi_{\text {Lia }}=\left(t_{L a}, b_{L a}\right)$. Once the top quark mass $m_{t}^{\text {sb }}$ is generated by the SSB, the quark-lepton interactions (5.8) introduce the ESB terms to the SD equations (mass-gap equations) for other fermions.

In order to show these ESB terms, we first approximate the SD equations to be selfconsistent mass gap-equations by neglecting perturbative gauge interactions and using the large $N_{c}$-expansion to the leading order, as indicated by figure 2 . The quark-lepton interactions (5.8), via the tadpole diagrams in figure 2, contribute to the tau lepton mass $m_{\tau}^{\mathrm{eb}}$ and tau neutrino mass $m_{\tau_{\nu}}^{\mathrm{eb}}$, provided the bottom quark mass $m_{b}^{\mathrm{eb}}$ and top quark mass $m_{t}^{\mathrm{sb}}$ are not zero. The latter $m_{t}^{\mathrm{sb}}$ is generated by the SSB, see section 4 . The former $m_{b}^{\mathrm{eb}}$ is generated by the ESB due to the $W^{ \pm}$-boson vector-like coupling and top-quark mass $m_{t}^{\text {sb }}$, see next section 5.2.

Corresponding to the tadpole diagrams in figure 2, the mass-gap equations of tau lepton and tau neutrino are given by

$$
\begin{aligned}
& m_{\nu_{\tau}}^{\mathrm{eb}}=\left(U_{L}^{\nu_{\tau} t} U_{R}^{t \nu_{\tau}}\right) 2 G m_{t}^{\mathrm{sb}} \frac{i}{(2 \pi)^{4}} \int d^{4} l\left[l^{2}-\left(m_{t}^{\mathrm{sb}}\right)^{2}\right]^{-1}=\left(U_{L}^{\nu_{\tau} t} U_{R}^{t \nu_{\tau}}\right)\left(1 / N_{c}\right) m_{t}^{\mathrm{sb}}, \\
& m_{\tau}^{\mathrm{eb}}=\left(U_{L}^{\tau b} U_{R}^{b \tau}\right) 2 G m_{b}^{\mathrm{eb}} \frac{i}{(2 \pi)^{4}} \int d^{4} l\left[l^{2}-\left(m_{b}^{\mathrm{eb}}\right)^{2}\right]^{-1}=\left(U_{L}^{\tau b} U_{R}^{b \tau}\right)\left(1 / N_{c}\right) m_{b}^{\mathrm{eb}}
\end{aligned}
$$

Here we use the self-consistent mass-gap equations of the bottom and top quarks (see eq. 2.1 and 2.2 in ref. [81])

$$
\begin{aligned}
& m_{b}^{\mathrm{eb}}=2 G N_{c} m_{b}^{\mathrm{eb}} \frac{i}{(2 \pi)^{4}} \int d^{4} l\left[l^{2}-\left(m_{b}^{\mathrm{eb}}\right)^{2}\right]^{-1}, \\
& m_{t}^{\mathrm{sb}}=2 G N_{c} m_{t}^{\mathrm{sb}} \frac{i}{(2 \pi)^{4}} \int d^{4} l\left[l^{2}-\left(m_{t}^{\mathrm{sb}}\right)^{2}\right]^{-1},
\end{aligned}
$$

and the definitions of Dirac quark, lepton and neutrino bare masses in eqs. (5.4)-(5.7). It is important to note the difference that eq. (5.12) is the mass-gap equation for the top-quark mass $m_{t}^{\mathrm{sb}}$ generated by the SSB, while eq. (5.11) is just a self-consistent mass-gap equation for the bottom-quark mass $m_{b}^{\mathrm{eb}} \neq 0$, as given by the tadpole diagram. The tau-neutrino mass $m_{\nu_{\tau}}^{\mathrm{eb}}$ and tau-lepton mass $m_{\tau}^{\mathrm{eb}}$ are not zero, if the top-quark mass $m_{t}^{\mathrm{sb}}$ and bottomquark mass $m_{b}^{\mathrm{eb}}$ are not zero. This is meant to the mass generation of tau neutrino and tau lepton due to the ESB terms introduced by the quark-lepton interactions (2.11), quark 
masses $m_{t}^{\mathrm{sb}}$ and $m_{b}^{\mathrm{eb}}$. On the other hand, if the tau-neutrino mass $m_{\nu_{\tau}}^{\mathrm{eb}}$ and tau-lepton mass $m_{\tau}^{\mathrm{eb}}$ are not zero, they also contribute to the self-consistent mass-gap equations for $m_{t}^{\mathrm{sb}}$ and $m_{b}^{\mathrm{eb}}$.

These discussions can be generalized to the three-family case by replacing $t \rightarrow t, c, u$ and $\nu_{\tau} \rightarrow \nu_{\tau}, \nu_{\mu}, \nu_{e}$ in eqs. (5.9) and (5.12); $b \rightarrow b, s, d$ and $\tau \rightarrow \tau, \mu, e$ in eqs. (5.10) and (5.11), and summing all contributions. All these self-consistent mass-gap equations are coupled together.

\section{2 $W^{ \pm}$-boson coupling to right-handed fermions}

In addition to the ESB terms due to quark-lepton interactions, the effective vertex of $W^{ \pm}$-boson coupling to right-handed fermions [97],

$$
\Gamma_{\mu}^{W}\left(p, p^{\prime}\right)=i \frac{g_{2}}{\sqrt{2}} \gamma_{\mu} P_{R} \Gamma^{W}\left(p, p^{\prime}\right)
$$

at the energy scale $\mathcal{E}$, also introduces the ESB terms to the Schwinger-Dyson equations. This is the main reason for the nontrivial bottom-quark mass $m_{b}$, once the top-quark mass $m_{t}$ is generated by the SSB [97]. This will be generalized to other fermions in section 6 .

Before leaving this section, we would like to mention that the vector-like feature of $W^{ \pm}$ boson coupling at high energy $\mathcal{E}$ is expected to have some collider signatures (asymmetry) on the decay channels of $W^{ \pm}$-boson into both left- and right-handed helicity states of two high-energy leptons or quarks $[88,94,95]$. The collider signatures should be more evident in high energies, where heavier fermions are produced. In fact, at the Fermilab Tevatron $p \bar{p}$ collisions the CDF [116] and D0 [117] experiments measured the forward-backward asymmetry in top-quark pair production

$$
A_{F B}=\frac{N_{t}(\cos \theta>0)-N_{t}(\cos \theta<0)}{N_{t}(\cos \theta>0)+N_{t}(\cos \theta<0)}=0.19 \pm 0.065 \text { (stat) } \pm 0.024 \text { (syst), }
$$

where the number $N_{t}(\cos \theta)$ of outgoing top quarks in the direction $\theta$ w.r.t. the incoming proton beam. This is larger than the asymmetry within the SM. In addition to the $s$ channel of one gauge boson $\left(\gamma, g, Z^{0}\right)$ exchange, the process $d\left(p_{1}\right) d\left(p_{2}\right) \rightarrow t\left(k_{1}\right) t\left(k_{2}\right)$, i.e., down-quark pair to top-quark pair, has the $t$-channel of one SM W-boson exchange. Its contributions to the asymmetry (5.14) and total $t \bar{t}$-production rate were studied [96] by assuming a new massive boson $W^{\prime}$ with left- and right-handed couplings $\left(g_{L}, g_{R}\right)$ to the top and down quarks. Performing the same analysis as that in ref. [96], we can explain the asymmetry $(5.14)$ by using the SM boson masses $\left(\approx M_{z}\right)$ and renormalized $S U_{L}(2)$ coupling $\bar{g}_{2}^{2}\left(M_{z}\right) \approx 0.45$ with $\left(g_{L}=1, g_{R}=\Gamma^{W} \approx \gamma_{w} \approx 0.57\right)$. The detailed analysis will be presented somewhere else. However, we want to point out that the analogous asymmetry should be also present in the $b \bar{b}$ channel, since the vector-like coupling (5.13) is approximately universal for all fermions [97].

\section{Schwinger-Dyson equations for fermion self-energy functions}

In order to understand how fermion masses are generated by the ESB and obey their RG equations, we are bound to study the Schwinger-Dyson (SD) equations for fermion selfenergy functions $\Sigma_{f}$. The SD equations are generalized from the third family [97] to the three families. 


\subsection{Chiral symmetry-breaking terms in SD equations}

In a vector-like gauge theory, SD equations for fermion self-energy functions were intensively studied in ref. [118-124]. In the Landau gauge, SD equations for quarks are given by

$$
\begin{array}{ll}
\Sigma_{f}(p)=m_{f}^{\mathrm{eb}}+m_{f}^{\mathrm{sb}} \delta_{f t}+3 \int_{p^{\prime}} \frac{V_{2 / 3}\left(p, p^{\prime}\right)}{\left(p-p^{\prime}\right)^{2}} \frac{\Sigma_{f}\left(p^{\prime}\right)}{p^{2}+\Sigma_{f}\left(p^{\prime}\right)} & f=u, c, t \\
\Sigma_{f^{\prime}}(p)=m_{f^{\prime}}^{\mathrm{eb}}+m_{f^{\prime}}^{\mathrm{sb}}+3 \int_{p^{\prime}} \frac{V_{-1 / 3}\left(p, p^{\prime}\right)}{\left(p-p^{\prime}\right)^{2}} \frac{\Sigma_{f^{\prime}}\left(p^{\prime}\right)}{p^{\prime 2}+\Sigma_{f^{\prime}}\left(p^{\prime}\right)} \quad f^{\prime}=d, s, b,
\end{array}
$$

where the integration $\int_{p^{\prime}} \equiv \int d^{4} p^{\prime} /(2 \pi)^{4}$ is up to the cutoff $\mathcal{E} . V_{2 / 3}\left(p, p^{\prime}\right)$ and $V_{-1 / 3}\left(p, p^{\prime}\right)$ are the vertex-functions of vector-like gauge theories. We neglect corrections to vertexfunctions of vector-like gauge interactions, for example, $V_{2 / 3}=(2 e / 3)^{2}$ and $V_{-1 / 3}=(e / 3)^{2}$ in the QED case.

In eqs. (6.1) and (6.2), only for the top quark the SSB-generated mass term $m_{t}^{\mathrm{sb}} \neq 0$, see the simplest mass-gap equation (5.12) and discussions in section 4, while for all other quarks the SSB-generated mass term $m_{f}^{\mathrm{sb}}=m_{f^{\prime}}^{\mathrm{sb}}=0$, see the discussions in section 4.1.

Instead, the bare mass terms $m_{f}^{\mathrm{eb}}$ and $m_{f^{\prime}}^{\mathrm{eb}}$ in eqs. (6.1) and (6.2) come from the ESB terms due to the quark-lepton interactions (2.12), see the self-consistent mass-gap equations (5.9)-(5.11), and the effective $W^{ \pm}$-boson coupling vertex (5.13). Since the vertex function $\Gamma^{W}\left(p, p^{\prime}\right)$ in eq. (5.13) does not vanish only for high energies, we approximately treat it as a boundary value at the scale $\mathcal{E}$

$$
\alpha_{w}=\alpha_{2}(\mathcal{E})\left(\gamma_{w} / \alpha_{c} \sqrt{2}\right), \quad \alpha_{2}(\mathcal{E})=\bar{g}_{2}^{2}(\mathcal{E}) / 4 \pi, \quad \gamma_{w}=\left.\Gamma^{W}\left(p, p^{\prime}\right)\right|_{p, p^{\prime} \rightarrow \mathcal{E}}
$$

where $\alpha_{c}=\pi / 3$, the $W$-contributions are approximately boundary terms in the integral SD equations (6.1) and (6.2), see figure 4 in ref. [97] for the third family.

We recall that in the SM the $W^{ \pm}$boson does not contribute to the SD equations for fermion self-energy functions $\Sigma_{f}$. However, due to the nontrivial vertex function (5.13), the $W^{ \pm}$gauge boson has the vector-like contributions to SD equations [32, 33]. These contributions not only introduce additional ESB terms, but also mix up SD equations for self-energy functions of different fermion fields via the CKM mixing matrix $U_{f f^{\prime}}=$ $\left(\mathcal{U}_{L}^{u \dagger} \mathcal{U}_{L}^{d}\right)_{f f^{\prime}}$ and the PMNS mixing matrix $U_{f f^{\prime}}^{\ell}=\left(\mathcal{U}_{L}^{\nu^{\dagger}} \mathcal{U}_{L}^{\ell}\right)_{f f^{\prime}}$

\subsection{Twelve coupled SD equations for SM quark and lepton masses}

Following the approach of ref. [118-124], we convert integral equations (6.1) and (6.2) to the following boundary value problems $\left(x=p^{2}, \alpha=e^{2} / 4 \pi\right)$ :

$$
\begin{aligned}
\frac{d}{d x}\left(x^{2} \Sigma_{f}^{\prime}(x)\right)+\frac{\alpha_{f}}{4 \alpha_{c}} \frac{x \Sigma_{f}(x)}{x+\Sigma_{f}^{2}(x)} & =0, \quad f=u, c, t \\
\mathcal{E}^{2} \Sigma_{f}^{\prime}\left(\mathcal{E}^{2}\right)+\Sigma_{f}\left(\mathcal{E}^{2}\right)-\delta_{f t} m_{t}^{\mathrm{sb}} & =\alpha_{w} \sum_{f^{\prime}=d, s, b}\left|U_{f f^{\prime}}\right|^{2} \Sigma_{f^{\prime}}\left(\mathcal{E}^{2}\right)+m_{f}^{\mathrm{eb}},
\end{aligned}
$$


and

$$
\begin{aligned}
\frac{d}{d x}\left(x^{2} \Sigma_{f^{\prime}}^{\prime}(x)\right)+\frac{\alpha_{f^{\prime}}}{4 \alpha_{c}} \frac{x \Sigma_{f^{\prime}}(x)}{x+\Sigma_{f^{\prime}}^{2}(x)} & =0, \quad f^{\prime}=d, s, b \\
\mathcal{E}^{2} \Sigma_{f^{\prime}}^{\prime}\left(\mathcal{E}^{2}\right)+\Sigma_{f^{\prime}}\left(\mathcal{E}^{2}\right) & =\alpha_{w} \sum_{f=u, c, t}\left|U_{f^{\prime} f}\right|^{2} \Sigma_{f}\left(\mathcal{E}^{2}\right)+m_{f^{\prime}}^{\mathrm{eb}},
\end{aligned}
$$

where the fine structure constant $\alpha_{f}\left(\alpha_{f^{\prime}}\right)$ corresponds to the quark sector $2 / 3(-1 / 3)$ and the QCD contributions are not explicitly shown.

Analogously, we obtain the following boundary value problems in the lepton sector:

$$
\begin{aligned}
\frac{d}{d x}\left(x^{2} \Sigma_{\ell_{\nu}}^{\prime}(x)\right) & =0, \quad \ell_{\nu}=\nu_{e}, \nu_{\mu}, \nu_{\tau} \\
\mathcal{E}^{2} \Sigma_{\ell_{\nu}}^{\prime}\left(\mathcal{E}^{2}\right)+\Sigma_{\ell_{\nu}}\left(\mathcal{E}^{2}\right) & =\alpha_{w} \sum_{\ell^{\prime}=e, \mu, \tau}\left|U_{\ell_{\nu} \ell^{\prime}}^{\ell}\right|^{2} \Sigma_{\ell^{\prime}}\left(\mathcal{E}^{2}\right)+m_{\ell_{\nu}}^{\mathrm{eb}},
\end{aligned}
$$

and

$$
\begin{aligned}
\frac{d}{d x}\left(x^{2} \Sigma_{\ell}^{\prime}(x)\right)+\frac{\alpha}{4 \alpha_{c}} \frac{x \Sigma_{\ell}(x)}{x+\Sigma_{\ell}^{2}(x)} & =0, \quad \ell=e, \mu, \tau \\
\mathcal{E}^{2} \Sigma_{\ell}^{\prime}\left(\mathcal{E}^{2}\right)+\Sigma_{\ell}\left(\mathcal{E}^{2}\right) & =\alpha_{w} \sum_{\ell_{\nu}^{\prime}}\left|U_{\ell \ell_{\nu}^{\prime}}^{\ell}\right|^{2} \Sigma_{\ell_{\nu}^{\prime}}(x)+m_{\ell}^{\mathrm{eb}},
\end{aligned}
$$

where $U_{\ell \ell_{\nu}^{\prime}}^{\ell}$ is the PMNS mixing matrix $U^{\ell}=\mathcal{U}_{L}^{\nu_{e} \dagger} \mathcal{U}_{L}^{e}$ of CKM-type in the lepton sector. The boundary conditions (6.5), (6.7), (6.9) and (6.11) are actually the mass-gap equations of quarks and leptons at the scale $\mathcal{E}$. Note that the quark-lepton interactions (2.12) have the contributions to the $m^{\mathrm{eb}}$-term in mass-gap equations (6.5), (6.7), (6.9) and (6.11), see also figure 2, therefore the quark and lepton mass-gap equations are coupled together. In total, these are twelve coupled and mixed SD equations of three quark and lepton families.

These twelve inhomogeneous SD equations admit massive solutions [118-125]

$$
\Sigma_{f}(p) \propto m_{f}\left(\frac{p^{2}}{m_{f}^{2}}\right)^{\gamma}, \quad m_{f} \leq p \leq \mathcal{E},
$$

where $\gamma \ll 1$ is the anomalous dimension of fermion mass operators, and running fermion masses at an infrared scale $\mu$ and mass-shell conditions read

$$
m_{f}(\mu)=\Sigma_{f}(\mu)=\bar{g}_{f}(\mu) v / \sqrt{2}, \quad m_{f}=\Sigma_{f}\left(m_{f}\right)(\mu)=\bar{g}_{f}\left(m_{f}\right) v / \sqrt{2},
$$

where $\bar{g}_{f}(\mu)$ is the corresponding Yukawa coupling, see more discussions in ref. [97].

\subsection{Realistic massive solutions}

Once the top-quark mass $m_{t}^{\mathrm{sb}}$ in eq. (6.5) is generated by the SSB, the SD equations (6.7) for $d, s, b$ quarks acquire inhomogeneous $\alpha_{w}$-terms via flavor mixing. Vice versa, once $d, s, b$ quarks are massive, the SD equations (6.5) for $u, c, t$ quarks acquire inhomogeneous $\alpha_{w^{-}}$ terms via flavor mixing as well. In the same way for the lepton sector, $\alpha_{w}$-terms due to the lepton-flavor mixing in eqs. (6.9) and (6.11) relate the massive solutions of charged 
leptons $e, \mu, \tau$ and neutrinos $\nu_{e}, \nu_{\mu}, \nu_{\tau}$. These inhomogeneous $\alpha_{w}$-terms are the ESB terms, in additional to the ESB mass terms $m_{f}^{\mathrm{eb}}, m_{f^{\prime}}^{\mathrm{eb}}, m_{\ell_{\nu}}^{\mathrm{eb}}$ and $m_{\ell}^{\mathrm{eb}}$ generated by quark-lepton interaction discussed in section 5.1.

These discussions show that the SSB generated top-quark mass $m_{t}^{\text {sb }}$ introduces the ESB terms into the SD equations of both quark and lepton sectors, which become inhomogeneous, completely coupled together and have nontrivial massive solutions. For instance, the top-quark mass $m_{t}$ introduces neutrino bare masses $m_{\ell_{\nu}}^{\mathrm{eb}}$ of eq. (5.1).

At the end of this section, it is worth noting that the top-quark mass $m_{t}$ is generated by the SSB with three Goldstone bosons, which become the longitudinal modes of massive $W^{ \pm}$and $Z^{0}$ gauge bosons, and the ESB for generating masses does not associate with Goldstone bosons. The top-quark mass $m_{t}$ is the unique origin of the ESB for generating all other fermion masses, therefore there is no any extra Goldstone boson in addition to those in the SSB channel of the top quark.

\section{The hierarchy spectrum of SM fermion masses}

In this section, we focus on approximately finding the qualitative fermion masses first for the third family $\left(\nu_{\tau}, \tau, t, b\right)$, then for the second family $\left(\nu_{\mu}, \mu, c, s\right)$ and the first family $\left(\nu_{e}, e, u, d\right)$, in order to understand what is the dominate contribution to each fermion mass and how the hierarchy of fermion masses is built in by the fermion-family mixing.

\subsection{The third fermion family}

This family is much more massive than the first and second fermion families in coupled SD equations. Therefore, we treat the massive solution $\left(m_{\nu_{\tau}}, m_{\tau}, m_{b}, m_{t}\right)$ of the third fermion family as a leading term and those for the first and second fermion families as perturbations in SD equations.

\subsubsection{Approximate fermion mass-gap equations for the third family}

In eqs. (6.5), (6.7), (6.9) and (6.11), we use eq. (6.12) to calculate the term $\mathcal{E}^{2} \Sigma_{i}^{\prime}\left(\mathcal{E}^{2}\right)=$ $\gamma \Sigma_{i}\left(\mathcal{E}^{2}\right) \ll \Sigma_{i}\left(\mathcal{E}^{2}\right)$, thus we neglect the term $\mathcal{E}^{2} \Sigma_{i}^{\prime}\left(\mathcal{E}^{2}\right)$ in these equations. At the scale $\mathcal{E}$, the top-quark bare mass $\Sigma_{t}\left(\mathcal{E}^{2}\right) \equiv m_{t}^{0} \approx m_{t}^{\text {sb }}$ introduces an explicit symmetry breaking term into SD equations for other fermions, and we define bare fermion masses $\Sigma_{f}\left(\mathcal{E}^{2}\right) \equiv$ $m_{f}^{0} \approx m_{f}^{\mathrm{eb}},\left(f=\nu_{\tau}, \tau, b\right)$ due to the ESB. Neglecting the contributions from the first and second fermion families, we approximately obtain the gap-equations (6.5), (6.7), (6.9) and (6.11) as follow,

$$
\begin{aligned}
m_{\nu_{\tau}}^{0} & \approx \alpha_{w}\left|U_{\tau \nu_{\tau}}^{\ell}\right|^{2} m_{\tau}^{0}+U_{L}^{\nu_{\tau} t} U_{R}^{t \nu_{\tau}} m_{t}^{0} / N_{c} \approx \mathcal{M}_{1} m_{t}^{0} / N_{c} \\
m_{\tau}^{0} & \approx \alpha_{w}\left|U_{\tau \nu_{\tau}}^{\ell}\right|^{2} m_{\nu_{\tau}}^{0}+U_{L}^{\tau b} U_{R}^{b \tau} m_{b}^{0} / N_{c} \approx \mathcal{M}_{2}(1 / 2) \alpha_{w} m_{t}^{0} \\
m_{t}^{0} & \approx \alpha_{w}\left|U_{t b}\right|^{2} m_{b}^{0}+U_{L}^{\nu_{\tau} t \dagger} U_{R}^{t \nu_{\tau} \dagger} m_{\nu_{\tau}}^{0}+m_{t}^{\mathrm{sb}} \approx m_{t}^{\mathrm{sb}} \\
m_{b}^{0} & \approx \alpha_{w}\left|U_{b t}\right|^{2} m_{t}^{0}+U_{L}^{\tau b \dagger} U_{R}^{b \tau \dagger} m_{\tau}^{0} \approx \mathcal{M}_{0}\left(N_{c} / 2\right) \alpha_{w} m_{t}^{0}
\end{aligned}
$$

where $\left|U_{t b}\right| \approx 1.03$ [114], $\left|U_{\tau \nu_{\tau}}^{\ell}\right| \approx(0.590 \rightarrow 0.776)$ [115]. The dominate contributions in the r.h.s. of these equations can be figured out. We obtain the approximate solution to 
eqs. (7.1) and (7.3), as well as the approximate solution to eqs. (7.2) and (7.4), which are given in the last step with

$$
\begin{aligned}
& \mathcal{M}_{0} \equiv\left|U_{b t}\right|^{2}+N_{c}^{-1}\left|U_{\tau \nu_{\tau}}^{\ell}\right|^{2}\left(U_{L}^{\nu_{\tau} t} U_{R}^{t \nu_{\tau}}\right)\left(U_{L}^{\tau b} U_{R}^{b \tau}\right) \approx\left|U_{b t}\right|^{2} \approx 1 \\
& \mathcal{M}_{1} \equiv U_{L}^{\nu_{\tau} t} U_{R}^{t \nu_{\tau}} \gg \alpha_{w}\left|U_{\tau \nu_{\tau}}^{\ell}\right|^{2}\left(m_{\tau}^{0} N_{c} / m_{t}^{0}\right) \sim \mathcal{O}\left(10^{-5}\right) \\
& \mathcal{M}_{2} \equiv\left|U_{b t}\right|^{2} U_{L}^{\tau b} U_{R}^{b \tau}+\left|U_{\tau \nu_{\tau}}^{\ell}\right|^{2} U_{L}^{\nu_{\tau} \tau} U_{R}^{t \nu_{\tau}}<2
\end{aligned}
$$

Equations (7.1)-(7.4) show that at the energy scale $\mathcal{E}$, the bare masses $m_{\nu_{\tau}}^{0}, m_{\tau}^{0}$ and $m_{b}^{0}$ are related to the bare mass $m_{t}^{0}$ from the SSB. The dominate contributions follow the following way: (i) the $\tau$-neutrino acquires its mass $m_{\nu_{\tau}}^{0}$ from the top-quark mass $m_{t}^{0}$ via the quark-lepton mixing (2.11) and figure 2 (right), (ii) the bottom-quark acquires its mass $m_{b}^{0}$ from the top-quark mass $m_{t}^{0}$ via the CKM mixing, (iii) the $\tau$-lepton acquires its mass $m_{\tau}^{0}$ from the bottom-quark mass $m_{b}^{0}$ via the quark-lepton mixing and $\tau$-neutrino mass $m_{\nu_{\tau}}^{0}$ via the PMNS mixing. These fermion bare masses $m_{f}^{0}$ due to the ESB at the scale $\mathcal{E}$ are in terms of the top-quark mass $m_{t}^{0}$ due to the SSB.

\subsubsection{Fermion masses and running Yukawa couplings}

These fermion bare masses $m_{f}^{0}$ evolve to their infrared masses $m_{f}(\mu)$, mainly follow the topquark one $m_{t}(\mu)$, apart from the energy-scale evolutions of the SM gauge interactions. In order to qualitatively calculate the infrared scale $m_{f}=m_{f}(\mu)$ as functions of the running scale $\mu$ in eq. (6.13) for each fermion " $f$ ", we neglect the corrections from perturbative gauge interactions and define the effective Yukawa couplings

$$
m_{b}(\mu)=\bar{g}_{b}(\mu) v / \sqrt{2}, \quad m_{\tau}(\mu)=\bar{g}_{\tau}(\mu) v / \sqrt{2}, \quad m_{\nu_{\tau}}(\mu)=\bar{g}_{\nu_{\tau}}(\mu) v / \sqrt{2},
$$

analogously to the top-quark mass $m_{t}(\mu)=\bar{g}_{t}(\mu) v / \sqrt{2}$. This means that effective fermion Yukawa couplings $\bar{g}_{f}(\mu)$ are functions of the top-quark one $\bar{g}_{t}(\mu)$.

Equations (7.1)-(7.4) become

$$
\begin{aligned}
m_{\nu_{\tau}}(\mu) & \approx \mathcal{M}_{1} m_{t}(\mu) / N_{c} \\
m_{\tau}(\mu) & \approx \mathcal{M}_{2}(1 / 2) \alpha_{w} m_{t}(\mu) \\
m_{b}(\mu) & \approx\left(N_{c} / 2\right) \alpha_{w} m_{t}(\mu) .
\end{aligned}
$$

Then, based on the top-quark mass-shell condition $m_{t}=\bar{g}_{t}\left(m_{t}\right) v / \sqrt{2}$ and experimental values of top and bottom quark masses: $m_{t}=m_{t}\left(m_{t}\right) \approx 173 \mathrm{GeV}$ and $m_{b}=m_{b}\left(m_{b}\right) \approx$ $4.2 \mathrm{GeV}$, as well as the Yukawa-coupling values $\bar{g}_{t}\left(m_{t}\right)=1.02$ and $\bar{g}_{t}\left(m_{b}\right)=1.29$ (figure 1 ), eq. (7.9) determines the approximately "universal" value in eq. (6.3) [97]

$$
\alpha_{w} \approx\left(2 / N_{c}\right)\left(\frac{m_{b}}{m_{t}}\right)\left[\frac{\bar{g}_{t}\left(m_{t}\right)}{\bar{g}_{t}\left(m_{b}\right)}\right] \approx 1.9 \times 10^{-2}\left(2 / N_{c}\right) .
$$

Equation $(6.3)$ gives $\gamma_{w} \approx 0.85\left(2 / N_{c}\right) \sim \mathcal{O}(1)$, where the value $\bar{g}_{2}^{2}(\mathcal{E}) \approx 0.42$. In this way, we approximately determine the finite part of vertex function $\Gamma^{W}\left(p, p^{\prime}\right)(5.13)$ or (6.3).

Using the Yukawa coupling $\bar{g}_{\tau}(\mu)(7.6)$ and $\bar{g}_{t}(\mu)$ (figure 1), we numerically solve eq. (7.8) and mass-shell condition $m_{\tau}=\bar{g}_{\tau}\left(m_{\tau}\right) v / \sqrt{2}$,

$$
m_{\tau} \approx 1.69 \mathrm{GeV}, \quad \text { for } \quad \mathcal{M}_{2} \approx 1.1
$$




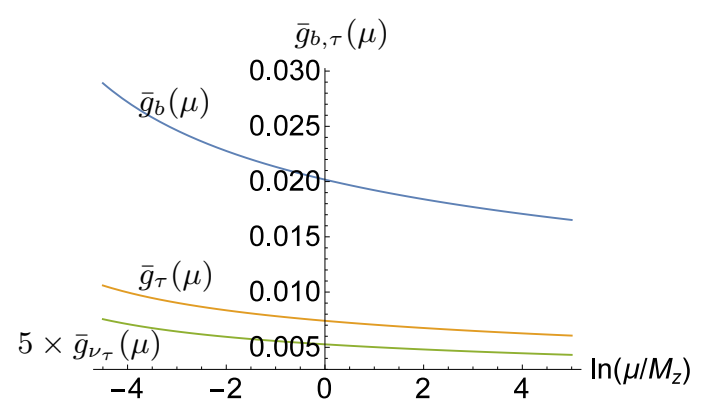

Figure 3. The Yukawa couplings $\bar{g}_{b}(\mu), \bar{g}_{\tau}(\mu)$ and $\bar{g}_{\nu_{\tau}}(\mu)$ in the range $1.0 \mathrm{GeV} \lesssim \mu \lesssim 13.5 \mathrm{TeV}$ for $\mathcal{M}_{2} \approx 1.1(7.11)$ and $\mathcal{M}_{1} \approx 3 \times 10^{-3}(7.12)$. Note that $m_{b, \tau, \nu_{\tau}}(\mu)=\bar{g}_{b, \tau, \nu_{\tau}}(\mu) v / \sqrt{2}$.

which is qualitatively consistent with the experimental value. Some contributions from the first and second fermion families should be expected. Analogously, using the Yukawa coupling $\bar{g}_{\nu_{\tau}}(\mu)(7.6)$ and $\bar{g}_{t}(\mu)$ (figure 1), we numerically calculate eq. (7.7) at $\mu=2 \mathrm{GeV}$ and obtain the neutrino Dirac mass

$$
m_{\nu_{\tau}} \approx 235.8 \mathrm{MeV}, \quad \text { for } \quad \mathcal{M}_{1}=U_{L}^{\nu_{\tau} t} U_{R}^{t \nu_{\tau}} \approx 3 \times 10^{-3} \text {. }
$$

Figure 3 shows the Yukawa couplings $\bar{g}_{\nu_{\tau}}(\mu), \bar{g}_{\tau}(\mu)$ and $\bar{g}_{b}(\mu)$, which are functions of $\bar{g}_{t}(\mu)$, see figure 1 . The variations of Yukawa couplings $\bar{g}_{b, \tau, \nu_{\tau}}(\mu)$ are very small over the energy scale $\mu$.

Equations (4.4) and (4.5) show that $\bar{g}_{t}(\mu)$ has received the contributions from gauge interactions $g_{1,2,3}(\mu)$ of the SM. This means that the RG-equations of these Yukawa couplings calculated are only valid in the high-energy region where the $\bar{g}_{3}(\mu)$ - and $\bar{g}_{2}(\mu)$-perturbative contributions to $\bar{g}_{t}(\mu)$ are taken into account. This is the reason that we adopt the point $\mu=2 \mathrm{GeV}$ to calculate $m_{\nu_{\tau}}$ (7.12), instead of using the mass-shell condition. The same reason will be for calculating at $\mu=2 \mathrm{GeV}$ the light fermion masses of the second and first families.

\subsection{The second fermion family}

In this section, we examine how the masses $m_{\nu_{\tau}, \tau, t, b}$ of the third fermion family introduce ESB terms into the SD equations of the second fermion family via SM gauge interactions and four-fermion interactions, leading to the mass generation of the second fermion family.

It is worthwhile to mention that at the lowest order (tree-level), SM neutral gaugebosons $\left(\gamma\right.$ and $\left.Z^{0}\right)$ interactions and four-fermion interactions (3.1) and (3.9) do not give rise to a 1PI vertex function of the interactions among three fermion families with the same electric charge $q=0,-1,2 / 3,-1 / 3$, as an example, the black blob in figure 4 . This indicates the separate conservations of $u$-quark, $c$-quark and $t$-quark numbers for the $q=2 / 3$ sector, and the same for other charged sectors $q=0,-1,-1 / 3$. As a result, the contributions of the 1PI self-energy functions, as shown in figure 4, to SD equations for fermion self-energy functions are negligible. 


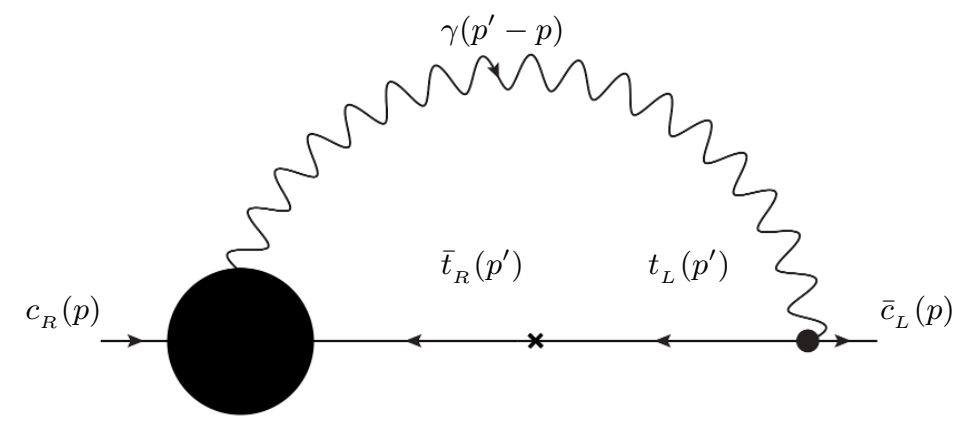

Figure 4. We adopt quarks $(t, c)$ as an example to illustrate a neutral gauge-boson $\gamma$ contribution to the fermion self-energy function $\Sigma_{c}(p)$ in terms of $\Sigma_{t}(p)$, the same diagrams for other quarks $(u, c, t)$ of $q=2 / 3$ charged sector, $(d, s, b)$ of $q=-1 / 3$ charged sector, as well as for other leptons $(e, \mu, \tau)$ of $q=-1$ charged sector, $\left(\nu_{e}, \nu_{\mu}, \nu_{\tau}\right)$ of $q=0$ neutral sector.

\subsubsection{Approximate fermion mass-gap equations of the second family}

Neglecting the contributions from the first fermion family, we assume that fermions in the second family mainly acquire their masses by ESB terms relating to fermion masses of the third family by the following ways: (i) family-mixing diagram, figure 4 in ref. [97], via $W^{ \pm}$-boson exchange at high-energy scale $\mathcal{E}$; (ii) Eq. (5.1) via tadpole diagrams figure 2 of quark-lepton interactions (2.11) or (2.12). Defining bare fermion masses $\Sigma_{f}\left(\mathcal{E}^{2}\right) \equiv m_{f}^{0} \approx$ $m_{f}^{\mathrm{eb}},\left(f=\nu_{\mu}, \mu, s, c\right)$, mass-gap equations (6.5), (6.7), (6.9) and (6.11) for the second fermion family can be approximately written as follow,

$$
\begin{aligned}
m_{\nu_{\mu}}^{0} \approx & \alpha_{w}\left|U_{\mu \nu_{\mu}}^{\ell}\right|{ }^{2} m_{\mu}^{0}+\alpha_{w}\left|U_{\tau \nu_{\mu}}^{\ell}\right|^{2} m_{\tau}^{0} \\
& +U_{L}^{\nu_{\mu} c} U_{R}^{c \nu_{\mu}} m_{c}^{0} / N_{c}+U_{L}^{\nu_{\mu} t} U_{R}^{t \nu_{\mu}} m_{t}^{0} / N_{c} \approx \mathcal{M}_{3} m_{t}^{0} \\
m_{\mu}^{0} \approx & \alpha_{w}\left(\left|U_{\mu \nu_{\mu}}^{\ell}\right|^{2} m_{\nu_{\mu}}^{0}+\left|U_{\mu \nu_{\tau}}^{\ell}\right|^{2} m_{\nu_{\tau}}^{0}\right)+U_{L}^{\mu s} U_{R}^{s \mu} m_{s}^{0} / N_{c}+U_{L}^{\mu b} U_{R}^{b \mu} m_{b}^{0} / N_{c} \\
\approx & \alpha_{w}\left(\left|U_{\mu \nu_{\mu}}^{\ell}\right|^{2} m_{\nu_{\mu}}^{0}+\left|U_{\mu \nu_{\tau}}^{\ell}\right|^{2} m_{\nu_{\tau}}^{0}\right)+\left(4 / N_{c}\right) \mathcal{M}_{6} m_{b}^{0} \\
m_{c}^{0} \approx & \alpha_{w}\left|U_{c s}\right|^{2} m_{s}^{0}+\alpha_{w}\left|U_{c b}\right|^{2} m_{b}^{0} \\
& +U_{L}^{\nu_{\mu} c \dagger} U_{R}^{c \nu_{\mu}^{\dagger}} m_{\nu_{\mu}}^{0}+U_{L}^{\nu_{\tau} c \dagger} U_{R}^{c \nu_{\tau} \dagger} m_{\nu_{\tau}}^{0} \approx\left(4 / N_{c}\right) \mathcal{M}_{4} m_{t}^{0} \\
m_{s}^{0} \approx & \alpha_{w}\left(\left|U_{s c}\right|^{2} m_{c}^{0}+\left|U_{s t}\right|^{2} m_{t}^{0}\right)+U_{L}^{\mu s \dagger} U_{R}^{s \mu \dagger} m_{\mu}^{0}+U_{L}^{\tau s \dagger} U_{R}^{s \tau \dagger} m_{\tau}^{0} \\
\approx & \alpha_{w}\left(\left|U_{s c}\right|^{2} m_{c}^{0}+\left|U_{s t}\right|^{2} m_{t}^{0}\right)+\mathcal{M}_{5} m_{b}^{0}
\end{aligned}
$$

where $\left|U_{c s}\right| \approx 0.986,\left|U_{c b}\right| \approx 4.1 \times 10^{-2}$ and $\left|U_{t s}\right| \approx 4.0 \times 10^{-2}$ [114], as well as $\left|U_{\tau \nu_{\mu}}^{\ell}\right| \approx$ $(0.614 \rightarrow 0.699),\left|U_{\mu \nu_{\tau}}^{\ell}\right| \approx(0.464 \rightarrow 0.713)$ and $\left|U_{\mu \nu_{\mu}}^{\ell}\right| \approx(0.441 \rightarrow 0.699)$ [115], and we use their central value for approximate calculations. The dominate contributions in the r.h.s. of these equations can be figured out. We obtain the approximate solution to eqs. (7.13) and (7.15), as well as the approximate solution to eqs. (7.14) and (7.16), which are given 
in the last step with

$$
\begin{aligned}
\mathcal{M}_{3} & \equiv \frac{1}{2}\left[U_{L}^{\nu_{\mu} t} U_{R}^{t \nu_{\mu}}+\left(U_{L}^{\nu_{\tau} t} U_{R}^{t \nu_{\tau}}\right)\left(U_{L}^{\nu_{\tau} c \dagger} U_{R}^{c \nu_{\tau} \dagger}\right)\left(U_{L}^{\nu_{\mu} c} U_{R}^{c \nu_{\mu}}\right)\right]=U_{L}^{\nu_{\mu} t} U_{R}^{t \nu_{\mu}} \\
\mathcal{M}_{4} & \equiv \frac{N_{c}}{8}\left[\frac{5}{N_{c}}\left(U_{L}^{\nu_{\tau} c \dagger} U_{R}^{c \nu_{\tau} \dagger}\right)\left(U_{L}^{\nu_{\tau} t} U_{R}^{t \nu_{\tau}}\right)+\left(U_{L}^{\nu_{\mu} c \dagger} U_{R}^{c \nu_{\mu} \dagger}\right)\left(U_{L}^{\nu_{\mu} t} U_{R}^{t \nu_{\mu}}\right)\right]=\left(U_{L}^{c t} U_{R}^{t c}\right) \\
\mathcal{M}_{5} & \equiv\left(U_{L}^{s \tau \dagger} U_{R}^{\tau s \dagger}\right)\left(U_{L}^{\tau b} U_{R}^{b \tau}\right)=\left(U_{L}^{s b} U_{R}^{b s}\right), \\
\mathcal{M}_{6} & \equiv\left(U_{L}^{\mu b} U_{R}^{b \mu}\right),
\end{aligned}
$$

where eq. (3.22) is used.

The dominate contributions in mass-gap equations (7.13)-(7.16) to the fermion masses are: (i) the $\nu_{\mu}$-neutrino and $c$-quark acquire their ESB masses $m_{\nu_{\mu}}^{0}$ and $m_{c}^{0}$ from the topquark mass $m_{t}^{0}$ via the quark-lepton interactions (3.21) between the third and second families, i.e., $\mathcal{M}_{3}$ and $\mathcal{M}_{4}$; (ii) the $s$-quark acquires it ESB mass $m_{s}^{0}$ via the CKM mixing and the quark-lepton interactions $\mathcal{M}_{5}$; (iii) the $\mu$-lepton acquires its ESB mass $m_{\mu}^{0}$ via the PMNS mixing and the quark-lepton interactions $\mathcal{M}_{6}$.

\subsubsection{Running fermion masses and Yukawa couplings}

Analogously to the discussion for the third fermion family from eqs. (7.1)-(7.4) to eqs. (7.7)(7.9), neglecting the perturbative corrections from the SM gauge interactions, and defining running fermion masses and Yukawa couplings

$$
\begin{aligned}
m_{\nu_{\mu}}(\mu) & =\bar{g}_{\nu_{\mu}}(\mu) v / \sqrt{2}, & m_{\mu}(\mu) & =\bar{g}_{\mu}(\mu) v / \sqrt{2}, \\
m_{c}(\mu) & =\bar{g}_{c}(\mu) v / \sqrt{2}, & m_{s}(\mu) & =\bar{g}_{s}(\mu) v / \sqrt{2},
\end{aligned}
$$

and the mass-gap equations at the scale $\mu$ are obtained by replacing $m_{f}^{0} \rightarrow m_{f}(\mu)$ in eqs. (7.13)-(7.16).

On the basis of eqs. (7.15) and (7.18) at the scale $\mu$ and the $c$-quark mass-shell condition $m_{c}=\bar{g}_{c}\left(m_{c}\right) v / \sqrt{2}$, as well as the results of the third family in section 7.1 , we numerically obtain

$$
m_{c} \approx 1.2 \mathrm{GeV}, \quad \text { for } \quad \mathcal{M}_{4}=\left(U_{L}^{c t} U_{R}^{t c}\right) \approx 3.6 \times 10^{-3}
$$

Using eqs. (7.13), (7.14), (7.16) and (7.18) at the scale $\mu$, we calculate the $\nu_{\mu}$-neutrino, light $s$-quark mass and $\mu$-muon mass at the scale $\mu=2 \mathrm{GeV}$,

$$
\begin{aligned}
m_{\nu_{\mu}} & \approx 2.4 \mathrm{MeV}, & & \text { for } \mathcal{M}_{3}=U_{L}^{\nu_{\mu} t} U_{R}^{t \nu_{\mu}} \approx 1.0 \times 10^{-5}, \\
m_{\mu} & \approx 121.5 \mathrm{MeV}, & & \text { for } \mathcal{M}_{6}=U_{L}^{\mu b} U_{R}^{b \mu} \approx 2.0 \times 10^{-2}, \\
m_{s} & \approx 91.2 \mathrm{MeV}, & & \text { for } \mathcal{M}_{5}=U_{L}^{s b} U_{R}^{b s} \approx 1.6 \times 10^{-2} .
\end{aligned}
$$

As a result, the Yukawa couplings $\bar{g}_{c}(\mu)$ and $\bar{g}_{\nu_{\mu}}(\mu)$ are shown in figure 5 , the Yukawa coupling $\bar{g}_{s}(\mu)$ and $\bar{g}_{\mu}(\mu)$ are shown in figure 6. The variations of Yukawa couplings $\bar{g}_{c, s, \mu, \nu_{\mu}}(\mu)$ are very small over the energy scale $\mu$. 


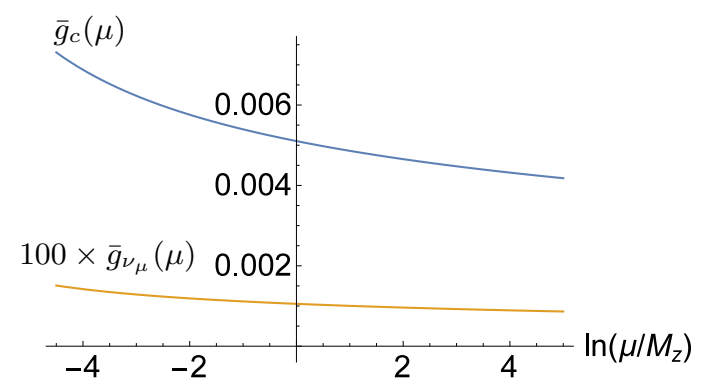

Figure 5. The Yukawa couplings $\bar{g}_{c}(\mu)$ and $\bar{g}_{\nu_{\mu}}(\mu)$ are plotted in the range $1.0 \mathrm{GeV} \lesssim \mu \lesssim 13.5 \mathrm{TeV}$ for $\mathcal{M}_{4} \approx 3.6 \times 10^{-3}(7.19)$ and $\mathcal{M}_{3} \approx 1.0 \times 10^{-5}(7.20)$. Note that $m_{c, \nu_{\mu}}(\mu)=\bar{g}_{c, \nu_{\mu}}(\mu) v / \sqrt{2}$.

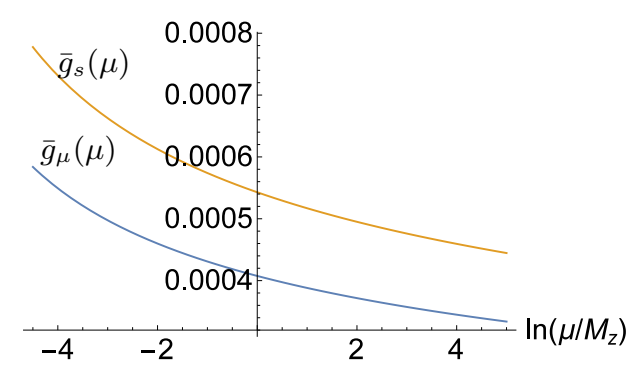

Figure 6. The Yukawa couplings $\bar{g}_{s}(\mu)$ and $\bar{g}_{\mu}(\mu)$ are plotted in the range $1.0 \mathrm{GeV} \lesssim \mu \lesssim 13.5 \mathrm{TeV}$ for $\mathcal{M}_{5}(7.22)$ and $\mathcal{M}_{6}(7.21)$. Note that $m_{s, \mu}(\mu)=\bar{g}_{s, \mu}(\mu) v / \sqrt{2}$.

In summary, the preliminary study (7.19)-(7.22) shows that the pattern of fermion masses in the second family can be consistently obtained by the pattern $\left(\mathcal{M}_{3,4,5,6}\right)$ of quark-lepton interactions and mixing between the third and second families. The scale $\mu$-evolution of masses and Yukawa couplings are functions of the top-quark one $\bar{g}_{t}(\mu)$, see figure 1.

\subsection{The first fermion family}

We turn to the masses and Yukawa couplings of the first fermion family. The coupled SD gap-equations receive ESB contributions from the third and second families, through the CKM and PMNS mixing as well as quark-lepton interactions between fermion families. As a result, the fermion masses of the first family are generated. Analogously to the calculations of the second family case, we neglect the perturbative contributions from gauge interactions and calculate the fermion masses at the scale $\mu=2 \mathrm{GeV}$.

\subsubsection{Approximate mass-gap equations of the first fermion family}

Analogously to eqs. (7.1)-(7.4) and eqs. (7.13)-(7.16) respectively for the third and second fermion family, Equations (6.5), (6.7), (6.9) and (6.11) for the first fermion family read,

$$
\begin{aligned}
m_{\nu_{e}}^{0} \approx & \alpha_{w}\left|U_{e \nu_{e}}^{\ell}\right|^{2} m_{e}^{0}+\alpha_{w}\left|U_{\mu \nu_{e}}^{\ell}\right|^{2} m_{\mu}^{0}+\alpha_{w}\left|U_{\tau \nu_{e}}^{\ell}\right|^{2} m_{\tau}^{0} \\
& +U_{L}^{\nu_{e} u} U_{R}^{u \nu_{e}} m_{u}^{0} / N_{c}+U_{L}^{\nu_{e} c} U_{R}^{c \nu_{e}} m_{c}^{0} / N_{c}+U_{L}^{\nu_{e} t} U_{R}^{t \nu_{e}} m_{t}^{0} / N_{c} \\
\approx & \left(11 / 2 N_{c}\right) U_{L}^{\nu_{e} t} U_{R}^{t \nu_{e}} m_{t}^{0}=\left(11 / 2 N_{c}\right) \mathcal{M}_{7} m_{t}^{0}
\end{aligned}
$$




$$
\begin{aligned}
m_{e}^{0} \approx & \alpha_{w}\left|U_{e \nu_{e}}^{\ell}\right|^{2} m_{\nu_{e}}^{0}+\alpha_{w}\left|U_{e \nu_{\mu}}^{\ell}\right|^{2} m_{\nu_{\mu}}^{0}+\alpha_{w}\left|U_{e \nu_{\tau}}^{\ell}\right|^{2} m_{\nu_{\tau}}^{0} \\
& +U_{L}^{e d} U_{R}^{d e} m_{d}^{0} / N_{c}+U_{L}^{e s} U_{R}^{s e} m_{s}^{0} / N_{c}+U_{L}^{e b} U_{R}^{b e} m_{b}^{0} / N_{c} \\
\approx & \left(N_{c} / 2\right) \alpha_{w}\left[\left|U_{e \nu_{e}}^{\ell}\right|^{2} m_{\nu_{e}}^{0}+\left|U_{e \nu_{\mu}}^{\ell}\right|^{2} m_{\nu_{\mu}}^{0}+\left|U_{e \nu_{\tau}}^{\ell}\right|^{2} m_{\nu_{\tau}}^{0}\right] \\
m_{u}^{0} \approx & \alpha_{w}\left|U_{u d}\right|^{2} m_{d}^{0}+\alpha_{w}\left|U_{u s}\right|^{2} m_{s}^{0}+\alpha_{w}\left|U_{u b}\right|^{2} m_{b}^{0} \\
& +U_{L}^{\nu_{e} u \dagger} U_{R}^{u \nu_{e} \dagger} m_{\nu_{e}}^{0}+U_{L}^{\nu_{\mu} u \dagger} U_{R}^{u \nu_{\mu} \dagger} m_{\nu_{\mu}}^{0}+U_{L}^{\nu_{\tau} u \dagger} U_{R}^{u \nu_{\tau} \dagger} m_{\nu_{\tau}}^{0} \\
\approx & \left(19 / 2 N_{c}\right) U_{L}^{u t} U_{R}^{t u} m_{t}^{0}=\left(19 / 2 N_{c}\right) \mathcal{M}_{8} m_{t}^{0}, \\
m_{d}^{0} \approx & \alpha_{w}\left|U_{d u}\right|^{2} m_{u}^{0}+\alpha_{w}\left|U_{d c}\right|^{2} m_{c}^{0}+\alpha_{w}\left|U_{d t}\right|^{2} m_{t}^{0} \\
& +U_{L}^{e d \dagger} U_{R}^{d e \dagger} m_{e}^{0}+U_{L}^{\mu d \dagger} U_{R}^{d \mu \dagger} m_{\mu}^{0}+U_{L}^{\tau d \dagger} U_{R}^{d \tau \dagger} m_{\tau}^{0} \\
\approx & \left(N_{c} / 2\right) \alpha_{w}\left[\left|U_{d u}\right|^{2} m_{u}^{0}+\left|U_{d c}\right|^{2} m_{c}^{0}+\left|U_{d t}\right|^{2} m_{t}^{0}\right]+(3 / 2) \mathcal{M}_{9} m_{b}^{0},
\end{aligned}
$$

where the CKM matrix elements $\left|U_{u d}\right| \approx 0.974,\left|U_{u s}\right| \approx 0.225,\left|U_{c d}\right| \approx 0.225,\left|U_{u b}\right| \approx$ $4.1 \times 10^{-3},\left|U_{t d}\right| \approx 8.4 \times 10^{-3}[114]$, as well as the PMNS matrix elements $\left|U_{e \nu_{e}}^{\ell}\right| \approx(0.801 \rightarrow$ $0.845),\left|U_{\mu \nu_{e}}^{\ell}\right| \approx(0.514 \rightarrow 0.580),\left|U_{\tau \nu_{e}}^{\ell}\right| \approx(0.137 \rightarrow 0.158),\left|U_{e \nu_{\mu}}^{\ell}\right| \approx(0.225 \rightarrow 0.517)$, $\left|U_{e \nu_{\tau}}^{\ell}\right| \approx(0.246 \rightarrow 0.529),\left|U_{\mu \nu_{\tau}}^{\ell}\right| \approx(0.464 \rightarrow 0.713)$ and $\left|U_{\mu \nu_{\mu}}^{\ell}\right| \approx(0.441 \rightarrow 0.699)$ [115]. The dominate contributions in the r.h.s. of these equations can be figured out. We obtain the approximate solution to eqs. (7.23) and (7.25), as well as the approximate solution to eqs. (7.24) and (7.26), which are given in the last step with

$$
\mathcal{M}_{7} \equiv U_{L}^{\nu_{e} t} U_{R}^{t \nu_{e}}, \quad \mathcal{M}_{8} \equiv U_{L}^{u t} U_{R}^{t u}, \quad \mathcal{M}_{9} \equiv U_{L}^{b d} U_{R}^{d b} .
$$

The dominate contributions are: (i) the $\nu_{e}$-neutrino acquires its mass $m_{\nu_{\mu}}^{0}$ from the $t$-quark mass $m_{t}^{0}$ via the quark-lepton interaction $\mathcal{M}_{7}$; (ii) the $u$-quark acquires its mass $m_{u}^{0}$ from the $t$-quark mass $m_{t}^{0}$ via the quark-lepton interaction $\mathcal{M}_{8}$; (iii) the $e$-lepton acquires its mass $m_{e}^{0}$ from the neutrino masses $m_{\nu_{e}}^{0}, m_{\nu_{\mu}}^{0}$ and $m_{\nu_{\tau}}^{0}$ via the PMNS mixing, which implies the approximate relation of light lepton masses and PMNS mixing angles; (iv) the $d$-quark dominantly acquires its mass $m_{d}^{0}$ from quark masses $m_{u}, m_{c}$ and $m_{t}$ via the CKM mixing, as well as a small contribution from the quark-lepton interaction $\mathcal{M}_{9}$, which implies the approximate relations of light quark masses and CKM mixing angles.

\subsubsection{Running fermion masses and Yukawa couplings}

Analogously to the discussion for the third fermion family from eqs. (7.1)-(7.4) to eqs. (7.7)(7.9), neglecting the perturbative corrections from the SM gauge interactions, and defining running fermion masses and Yukawa couplings

$$
\begin{aligned}
m_{\nu_{e}}(\mu) & =\bar{g}_{\nu_{e}}(\mu) v / \sqrt{2}, & & m_{e}(\mu)=\bar{g}_{e}(\mu) v / \sqrt{2}, \\
m_{u}(\mu) & =\bar{g}_{u}(\mu) v / \sqrt{2}, & & m_{d}(\mu)=\bar{g}_{d}(\mu) v / \sqrt{2},
\end{aligned}
$$

and the gap-equations at the scale $\mu$ are obtained by replacing $m_{f}^{0} \rightarrow m_{f}(\mu)$ in eqs. (7.23)(7.26). On the basis of eqs. (7.23), (7.25) and (7.28) at the scale $\mu$, we numerically calculate the $\nu_{e}, e, u$ - and $d$-quark masses at $\mu=2 \mathrm{GeV}$

$$
\begin{aligned}
m_{\nu_{e}} & \approx 4.3 \mathrm{KeV}, \quad \text { for } \quad \mathcal{M}_{7}=U_{L}^{\nu_{e} t} U_{R}^{t \nu_{e}} \approx 1.0 \times 10^{-7} \\
m_{u} & \approx 2.2 \mathrm{MeV}, \quad \text { for } \quad \mathcal{M}_{8}=\left(U_{L}^{u t} U_{R}^{t u}\right) \approx 3.0 \times 10^{-6}
\end{aligned}
$$




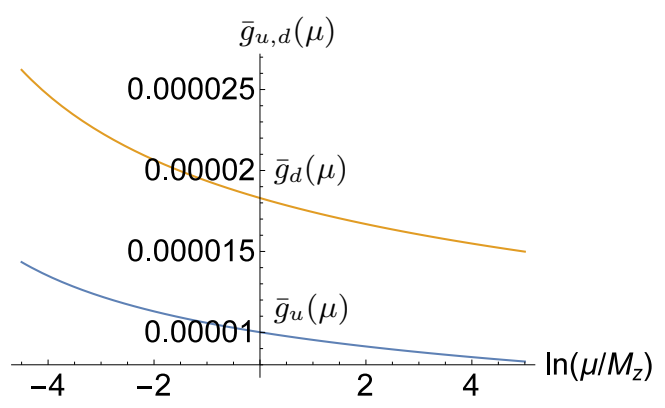

Figure 7. The Yukawa couplings $\bar{g}_{u}(\mu)$ and $\bar{g}_{d}(\mu)$ are plotted in the range $1.0 \mathrm{GeV} \lesssim \mu \lesssim 13.5 \mathrm{TeV}$ for $\mathcal{M}_{8}(7.30)$ and $\mathcal{M}_{9}$ (7.31). Note that $m_{u, d}(\mu)=\bar{g}_{u, d}(\mu) v / \sqrt{2}$.

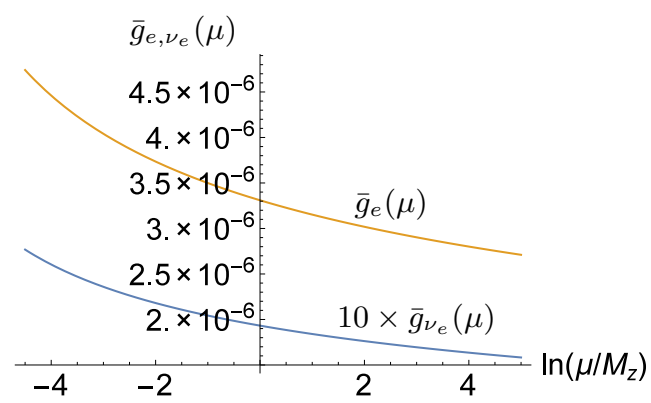

Figure 8. The Yukawa couplings $\bar{g}_{e}(\mu)$ and $\bar{g}_{\nu_{e}}(\mu)$ are plotted in the range $1.0 \mathrm{GeV} \lesssim \mu \lesssim 13.5 \mathrm{TeV}$ for $\mathcal{M}_{7}$ (7.29). Note that $m_{e, \nu_{e}}(\mu)=\bar{g}_{e, \nu_{e}}(\mu) v / \sqrt{2}$.

$$
\begin{aligned}
& m_{d} \approx 4.1 \mathrm{MeV}, \quad \text { for } \quad \mathcal{M}_{9}=\left(U_{L}^{b d} U_{R}^{d b}\right) \approx 4.0 \times 10^{-4} \\
& m_{e} \approx 0.7 \mathrm{MeV},
\end{aligned}
$$

and the Yukawa couplings $\bar{g}_{u}(\mu)$ and $\bar{g}_{d}(\mu)$, see figure 7 , and $\bar{g}_{e}(\mu)$ and $\bar{g}_{\nu_{e}}(\mu)$, see figure 8 . The variations of Yukawa couplings $\bar{g}_{u, d, e, \nu_{e}}(\mu)$ are very small over the energy scale $\mu$.

\subsection{Summary and discussion}

We show that the top-quark mass and Yukawa coupling $m_{t}(\mu)=\bar{g}_{t}(\mu) v / \sqrt{2}$, which is originated from the SSB, inevitably introduce the inhomogeneous (ESB) terms into the SD equations for other fermion masses via the fermion-family mixing due to the quarklepton interactions and the $W^{ \pm}$-boson vector-like vertex (CKM and PMNS mixing) at high energies. As a consequence, this leads to the generations of other fermion masses by the ESB mechanism, and their Yukawa couplings $\left(m_{f}(\mu)=\bar{g}_{f}(\mu) v / \sqrt{2}\right)$ are functions of the top-quark Yukawa coupling $\bar{g}_{t}(\mu)$, figure 1 . We approximately analyze the coupled SD gap-equations for the fermion masses and Yukawa couplings of the third, second and the first family of the SM. With the knowledge of the CKM and PMNS matrices, as well as the fermion mass spectra, we try to identify the dominate ESB contributions to the SD gap-equations, and approximately find their masses, consistently with the fermion-family mixing parameters $\mathcal{M}_{i}$. We have checked that the contributions from perturbative gauge interactions are negligible, compared with the essential contributions due to the fermionfamily mixing. As qualitative and preliminary results, without any drastic fine-tuning we 


\begin{tabular}{|c|c|c|}
\hline$m_{\nu_{e}} \approx 4.3 \times 10^{-6} \mathrm{GeV}$ & $m_{\nu_{\mu}} \approx 2.4 \times 10^{-3} \mathrm{GeV}$ & $m_{\nu_{\tau}} \approx 2.4 \times 10^{-1} \mathrm{GeV}$ \\
\hline$m_{e} \approx 7.0 \times 10^{-4} \mathrm{GeV}$ & $m_{\mu} \approx 1.2 \times 10^{-1} \mathrm{GeV}$ & $m_{\tau} \approx 1.7 \times 10^{0} \mathrm{GeV}$ \\
\hline$m_{u} \approx 2.2 \times 10^{-3} \mathrm{GeV}$ & $m_{c} \approx 1.3 \times 10^{0} \mathrm{GeV}$ & $m_{t} \approx 1.7 \times 10^{2} \mathrm{GeV}$ \\
\hline$m_{d} \approx 4.1 \times 10^{-3} \mathrm{GeV}$ & $m_{s} \approx 9.1 \times 10^{-2} \mathrm{GeV}$ & $m_{b} \approx 4.2 \times 10^{0} \mathrm{GeV}$ \\
\hline
\end{tabular}

Table 1. We present our qualitative result of the hierarchy spectrum of 12 SM-fermion masses, which seems to be consistent with the SM. The top-quark mass is generated by the SSB, and others by the ESB attributed to the top-quark mass and family-mixing. All masses are calculated at $\mu=2 \mathrm{GeV}$, except $m_{t}, m_{b}$ and $m_{\tau}$ calculated by their mass-shell conditions.

approximately obtain the hierarchy pattern of 12 SM-fermion masses, see table 1 , and their Yukawa couplings, consistently with the parameter $\alpha_{w}(6.3)$ and the hierarchy pattern of 9 family-mixing parameters $\mathcal{M}_{i}$.

It is energetically favorable that the SSB solely occurs for the $\bar{t} t$-channel (1.1) generating the top-quark mass and three Goldstone modes only. The SSB realizes the approximate ground state (vacuum), in which the pattern of fermion masses is $m_{t} \neq 0$ and $m_{f \neq t}=0$. However, this SSB generated vacuum alignment is re-arranged to the real ground states, where the real hierarchy pattern (table 1) is realized. Such rearrangement is due to the nontrivial ESB terms in the SD gap-equations for fermion masses, so that fermions become massive $m_{t} \gg m_{f \neq t} \neq 0$. These ESB terms are introduced by the top-quark mass and fermion-family mixing matrices in the two ways: (i) the fermion-family-mixing matrices (3.8) and (3.17) including the CKM and PMNS matrices introduce the ESB terms, due to the vector-like coupling $\alpha_{w}(5.13)$ and $(6.3)$ of the $W^{ \pm}$-boson at high energies $\mathcal{E}$ (see preliminary study $[32,33]$ ); (ii) the quark-lepton-family mixing matrices (3.22) introduce the ESB terms, due to the quark-lepton interactions (3.21) at high energies $\mathcal{E}$. It is expected that the ESB terms perturbatively re-arrange the SSB generated vacuum alignment, because of the small coupling $\alpha_{w}$ and fermion-family-mixing matrix elements. The table 1 shows that the following relations between (i) neutrino Dirac masses and charged $(2 / 3)$ quark masses; (ii) charged lepton and charged (-1/3) quark masses;

$$
\begin{array}{r}
m_{\nu_{\tau}}: m_{\nu_{\mu}}: m_{\nu_{e}} \approx m_{t}: m_{c}: m_{u} \approx 10^{-5}: 10^{-2}: 1, \\
m_{\tau}: m_{\mu}: m_{e} \approx m_{b}: m_{s}: m_{d} \approx 10^{-4}: 10^{-2}: 1 .
\end{array}
$$

In conclusion, the spectrum of fermion masses, i.e., the structure of eigenvalues of fermion mass matrices mainly depends on the ESB terms that relats to the unitary matrices or mixing matrices between three fermion-flavor families and four families of fermions with different electric charge. We cannot theoretically determine these matrices, except for adopting those CKM- and PMNS-matrix elements already experimentally measured. If these fermion-family mixing-matrix elements are small deviations from triviality, namely the hierarchy pattern likes the observed CKM matrix, the pattern of fermion masses is hierarchy, and vice versa. In this article, the hierarchy pattern of fermion masses (Yukawa couplings) is obtained consistently with the hierarchy pattern of fermion-family mixingmatrix elements. It should be mentioned that both of them are equally the basic parameters of the Nature, and they are closely related each other by the symmetries and/or dynamics 
of the fundamental theory. Some relations between them are given in this article, however more fundamental relations are expected in the framework of unification theories, e.g., $\mathrm{SO}(10)$-theory. For the light quarks and leptons, they acquire their masses dominantly from the ESB terms of the $W^{ \pm}$-boson coupling $\alpha_{w}$-terms associating with either CKM or PMNS matrix. This implies that there are the approximate relations of light quark/lepton masses and CKM/PMNS mixing angles, as intensively studied in literature.

It should be emphasized that we have at the infrared scale $12 \mathrm{SD}$ equations for 12 SM fermion masses coupled together via the fermion-family-mixing matrices (3.8), (3.17) and (3.22), which are unknown except the well (poor) known CKM (PMNS) matrix. These mixing matrices have to be understood in a UV-fundamental theory symmetrically unifying not only gauge interactions but also three fermion families. In this sense, fermion mixing matrices are even more fundamental than fermion masses. Their values, mixing matrix elements and fermion masses in unit of the top-quark mass, are related and determined upon the chiral-symmetry-breaking ground state of the UV-fundamental theory. The presented results only show that the known hierarchical masses $\left(10^{0}-10^{-8}\right)$ of 12 SM Dirac fermions are related to the hierarchical pattern of 9 fermion-family mixing parameters $\mathcal{M}_{i}$ $\left(10^{0}-10^{-7}\right)$ of eqs. $(7.5),(7.17)$ and $(7.27)$. Since we have not understood the hierarchical mixing-matrix pattern of the UV-fundamental theory, the hierarchical fermion masses are not ultimately explained. It should be also emphasized that the presented results are preliminarily qualitative, and far from being quantitatively compared with the SM fermion masses and precision tests of e.g., Yukawa couplings. Due to the fact that 12 coupled SD mass-gap equations depend on not only poorly known and totally unknown family-mixing parameters, but also running gauge couplings, the quantitative study of solving these SD equations is a difficult and challenging task. These results could be quantitatively improved, if one would be able to solve coupled SD equations by using a numerical approach in future. Our goal in this article is to present an insight into a possible scenario and understanding of the origins and hierarchy spectrum of fermion masses in the SM without drastic fine-tuning.

In the next section, we will relabel neutrino Dirac mass $m_{\nu}$ by $m_{\nu}^{D}$, discuss three heavy sterile Majorana neutrinos $\left(\nu_{R}^{f}+\nu_{R}^{f c}\right)$ and three light gauged Majorana neutrinos $\left(\nu_{L}^{f}+\nu_{L}^{f c}\right)$ in terms of their Dirac masses $m_{\nu}^{\mathrm{D}}$ and Majorana masses $m_{\nu}^{\mathrm{M}}$.

\section{Neutrino sector}

On the basis of Dirac neutrino mass eigenstates and masses calculated (see table 1) in previous sections, as well as some experimental results of neutrino oscillations, we calculate the mass-spectra of gauged and sterile neutrinos by taking into account the Majorana masses generated by the spontaneous symmetry breaking of the global $U_{\text {lepton }}(1)$ symmetry for the lepton-number conservation.

\subsection{Spontaneous symmetry breaking of $U_{\text {lepton }}(1)$ symmetry}

In the four-fermion operators (3.9) of the lepton sector, the last term reads

$$
-G \sum_{f f^{\prime}}\left[\left(\bar{\nu}_{R}^{f c} \nu_{R}^{f^{\prime}}\right)\left(\bar{\nu}_{R}^{f^{\prime}} \nu_{R}^{f c}\right)\right]
$$


where the conjugate fields of sterile Wely neutrinos $\nu_{R}^{f}$ are given by $\nu_{R}^{f c}=i \gamma_{2}\left(\nu_{R}^{f}\right)^{*}$. This four-fermion operator preserves the global $U_{\text {lepton }}(1)$-symmetry for the lepton-number conservation. Similarly to the discussions of the SSB mechanism for the generation of top-quark mass in section 4, the four-fermion operator (8.1) can generate a mass term of Majorana type, since the family index " $f$ " is summed over as the color index " $a$ " and the family number $N_{f}=3$ plays the similar role as the color number $N_{c}$ in the $\langle\bar{t} t\rangle$-condensate (4.1). We notice that the lepton-number is conserved in the ground state (vacuum state) realized by the SSB of the SM chiral gauge symmetries, whereas the lepton-number is not conserved in the ground state realized by the spontaneous symmetry breaking of the global $U_{\text {lepton }}(1)$-symmetry of the Lagrangian (8.1).

On the basis of the mass eigenstates, the spontaneous symmetry breaking of the $U_{\text {lepton }}(1)$-symmetry generates the masses of Majorana type

$$
m^{M}=\sum_{f=1,2,3} m_{f}^{M}, \quad m_{f}^{M}=-G\left\langle\bar{\nu}_{R}^{f c} \nu_{R}^{f}\right\rangle,
$$

together with a sterile massless Goldstone boson, i.e. the pseudoscalar bound state

$$
\phi^{M}=\sum_{f=1,2,3}\left\langle\bar{\nu}_{R}^{f c} \gamma_{5} \nu_{R}^{f}\right\rangle
$$

and a sterile massive scalar particle, i.e. the scalar bound state

$$
\phi_{H}^{M}=\sum_{f=1,2,3}\left\langle\sum_{f} \bar{\nu}_{R}^{f c} \nu_{R}^{f}\right\rangle
$$

both of them carry two units of the lepton number. The sterile neutrino mass $m^{M}$ and sterile scalar particle mass $m_{H}^{M}$ satisfy the mass-shell conditions,

$$
m^{M}=\bar{g}_{\text {sterile }}\left(m^{M}\right) v_{\text {sterile }} / \sqrt{2}, \quad\left(m_{H}^{M}\right)^{2} / 2=\tilde{\lambda}_{\text {sterile }}\left(m_{H}^{M}\right) v_{\text {sterile }}^{2}
$$

where $\bar{g}_{\text {sterile }}\left(\mu^{2}\right)$ and $\tilde{\lambda}_{\text {sterile }}\left(\mu^{2}\right)$ obey the same RG equations (absence of gauge interactions) of eqs. (4.3), (4.4) and (4.5), as well as the boundary conditions (8.5). However, we cannot determine the solutions $\bar{g}_{\text {sterile }}\left(\mu^{2}\right)$ and $\tilde{\lambda}_{\text {sterile }}\left(\mu^{2}\right)$, since the energy scale $v_{\text {sterile }}$ of boundary conditions (8.5) are unknown. The electroweak scale $v$ is determined by the gauge-boson masses $M_{W}$ and $M_{Z}$ experimentally measured, the scale $v_{\text {sterile }}$ needs to be determined by the sterile neutrino masses $m_{i}^{M}$ and the sterile scalar particle mass $m_{H}^{M}$. In fact, the scale $v_{\text {sterile }}$ represents the energy scale of the lepton-number violation.

\subsection{Gauged and sterile Majorana neutrino masses}

The SSB and ESB of the SM chiral gauge symmetries, as well as the spontaneous symmetry breaking of the $U_{\text {lepton }}(1)$-symmetry result in the following bilinear Dirac and Majorana mass terms

$$
m_{f}^{D} \bar{\nu}_{L}^{f} \nu_{R}^{f}+m_{f}^{M} \bar{\nu}_{R}^{f c} \nu_{R}^{f}+\text { h.c. }
$$


in terms of neutrino mass eigenstates $\nu_{L}^{f}$ and $\nu_{R}^{f}$ in the $f$-th fermion family, see eqs. (3.14) and (3.15). Following the usual approach [112, 113], diagonalizing the $2 \times 2$ mixing matrix (8.6) in terms of the neutrino and sterile neutrino mass eigenstates of the family " $f=1,2,3$ ", we obtains two mass eigenvalues

$$
\begin{aligned}
& M_{f}^{g}=\frac{1}{2}\left\{m_{f}^{M}-\left[\left(m_{f}^{M}\right)^{2}+\left(m_{f}^{D}\right)^{2}\right]^{1 / 2}\right\} \\
& M_{f}^{s}=\frac{1}{2}\left\{m_{f}^{M}+\left[\left(m_{f}^{M}\right)^{2}+\left(m_{f}^{D}\right)^{2}\right]^{1 / 2}\right\} .
\end{aligned}
$$

This corresponds to two mass eigenstates: three light gauged Majorana neutrinos (four components)

$$
\nu_{g}^{f}=\nu_{L}^{f}+\nu_{L}^{f c}, \quad E_{g}^{f}=\left[p^{2}+\left(M_{f}^{g}\right)^{2}\right]^{1 / 2}, \quad M_{f}^{g} \approx\left(m_{f}^{D}\right)^{2} / 4 m_{f}^{M},
$$

and three heavy sterile Majorana neutrinos (four components)

$$
\nu_{s}^{f}=\nu_{R}^{f}+\nu_{R}^{f c}, \quad E_{s}^{f}=\left[p^{2}+\left(M_{f}^{s}\right)^{2}\right]^{1 / 2}, \quad M_{f}^{s} \approx m_{f}^{M} .
$$

where $p$ stands for neutrino momentum, corresponding velocity $v_{p}$. The mixing angles between gauged and sterile Majorana neutrinos are

$$
2 \theta_{f}=\tan ^{-1}\left(m_{f}^{D} / m_{f}^{M}\right) \approx\left(m_{f}^{D} / m_{f}^{M}\right) \ll 1,
$$

The previously obtained Dirac masses $m_{f} \equiv m_{f}^{D}$ have the structure of hierarchy (see table 1). The discussions after eq. (3.19) show that the Majorana masses $m_{f}^{M}$ are expected to have a hierarchy structure relating to the one of Dirac masses $m_{f}^{D}$. ${ }^{3}$ This indicates the normal hierarchy structure of neutrino mass spectrum: Dirac neutrino masses $m_{1}^{D}<m_{2}^{D}<$ $m_{3}^{D}$, sterile Majorana neutrino masses $m_{1}^{M}<m_{2}^{M}<m_{3}^{M}$ (8.10) and gauged Majorana neutrino masses $M_{1}^{g}<M_{2}^{g}<M_{3}^{g}(8.9)$, i.e.,

$$
\left(m_{1}^{D}\right)^{2} / 4 m_{1}^{M}<\left(m_{2}^{D}\right)^{2} / 4 m_{2}^{M}<\left(m_{3}^{D}\right)^{2} / 4 m_{3}^{M} .
$$

Moreover, due to the absence of observed lepton-violating processes up to the electroweak scale and the smallness of gauged neutrino masses, it is nature to assume that the neutrino Majorana masses are much larger than their Dirac masses $m_{f}^{M} \gg m_{f}^{D}$, i.e., the energy scale $v_{\text {sterile }}$ of the lepton-number violation is much larger than the electroweak scale $v$.

\subsection{Flavor oscillations of gauged Majorana neutrinos}

We first discuss the family-flavor oscillations of three light gauged Majorana neutrinos (8.9) in the usual framework. They are described by the PMNS mixing matrix $U_{L}^{\ell}=\mathcal{U}_{L}^{\nu \dagger} \mathcal{U}_{L}^{\ell}$, the mass and mass-squared differences of gauged Majorana neutrino mass-eigenstates $\left(f, f^{\prime}=\right.$

\footnotetext{
${ }^{3}$ In ref. [107], we assume that the Majorana masses $m_{f}^{M}$ are approximately equal (degenerate) $m_{i}^{M} \approx$ $m^{M} / 3$, since there is not any preferential $i$-th component of the condensate $\left\langle\bar{\nu}_{R}^{i c} \nu_{R}^{i}\right\rangle$. This is not correct because of the non-trivial chiral transformation $\mathcal{U}_{R}^{\nu}$ in eqs. (3.14) and (3.15).
} 


\begin{tabular}{|c|c|c|c|}
\hline Dirac mass & $m_{1}^{D} \approx 4.3 \times 10^{-6} \mathrm{GeV}$ & $m_{2}^{D} \approx 2.4 \times 10^{-3} \mathrm{GeV}$ & $m_{3}^{D} \approx 2.4 \times 10^{-1} \mathrm{GeV}$ \\
\hline Majorana mass & $m_{1}^{M} \approx 1.7 \times 10^{2} \mathrm{GeV}$ & $m_{2}^{M} \approx 1.7 \times 10^{5} \mathrm{GeV}$ & $m_{3}^{M} \approx 2.9 \times 10^{8} \mathrm{GeV}$ \\
\hline sterile neutrino mass & $M_{1}^{s} \approx 1.7 \times 10^{2} \mathrm{GeV}$ & $M_{2}^{s} \approx 1.7 \times 10^{5} \mathrm{GeV}$ & $M_{3}^{s} \approx 2.9 \times 10^{8} \mathrm{GeV}$ \\
\hline gauged neutrino mass & $M_{1}^{g} \approx 2.8 \times 10^{-5} \mathrm{eV}$ & $M_{2}^{g} \approx 8.4 \times 10^{-3} \mathrm{eV}$ & $M_{3}^{g} \approx 5.0 \times 10^{-2} \mathrm{eV}$ \\
\hline
\end{tabular}

Table 2. Spectra of neutrino Dirac masses $m_{f}^{D}$, neutrino Majorana masses $m_{f}^{M}$, sterile Majorana neutrino masses $M_{f}^{s} \approx m_{f}^{M}$ and gauged Majorana neutrino masses $M_{f}^{g} \approx\left(m_{f}^{D}\right)^{2} / 4 m_{f}^{M}$. Here we label three flavor-families by using notation $f=1,2,3$ instead of $f=\nu_{e}, \nu_{\mu}, \nu_{\tau}$.

$1,2,3)$, which are calculated by using eq. (8.7)

$$
\begin{aligned}
& \Delta M_{f f^{\prime}}^{g} \equiv M_{f}^{g}-M_{f^{\prime}}^{g} \approx\left[\frac{\left(m_{f^{\prime}}^{D}\right)^{2}}{4 m_{f^{\prime}}^{M}}\right]-\left[\frac{\left(m_{f}^{D}\right)^{2}}{4 m_{f}^{M}}\right], \\
& \Delta M_{f f^{\prime}}^{g 2} \equiv\left(M_{f}^{g}\right)^{2}-\left(M_{f^{\prime}}^{g}\right)^{2} \approx\left[\frac{\left(m_{f}^{D}\right)^{2}}{4 m_{f}^{M}}\right]^{2}-\left[\frac{\left(m_{f^{\prime}}^{D}\right)^{2}}{4 m_{f^{\prime}}^{M}}\right]^{2},
\end{aligned}
$$

Equation (8.13) is up to the order $\mathcal{O}\left\{\left(m_{f}^{D}\right)^{2} / 4 m_{f}^{M}\right\}$, and eq. (8.14) is up to the order $\mathcal{O}\left\{\left[\left(m_{f}^{D}\right)^{2} / 4 m_{f}^{M}\right]^{2}\right\}$. The oscillating probability from the flavor $\nu_{\alpha}^{g}$ to the flavor $\nu_{\beta}^{g}$ reads

$$
P_{\nu_{\alpha}^{g} \longleftrightarrow \nu_{\beta}^{g}}(t)=\sum_{f f^{\prime}}\left(U_{L}^{\ell}\right)_{\alpha f}^{*}\left(U_{L}^{\ell}\right)_{\beta f}\left(U_{L}^{\ell}\right)_{\alpha f^{\prime}}\left(U_{L}^{\ell}\right)_{\beta f^{\prime}}^{*} \exp \left[-i\left(E_{g}^{f}-E_{g}^{f^{\prime}}\right) t\right] .
$$

The large oscillating lengths of relativistic and non-relativistic gauged neutrinos are given by

$$
\begin{array}{ll}
L_{g}^{f f^{\prime}}=\frac{2 \pi}{\left(E_{g}^{f}-E_{g}^{f^{\prime}}\right)} \approx \frac{2 \pi(2 p)}{\Delta M_{f f^{\prime}}^{g 2}} \quad \text { for } \quad p \gg \frac{\left(m_{f}^{D}\right)^{2}}{4 m_{f}^{M}}, \quad\left(f \rightarrow f^{\prime}\right), \\
L_{g}^{f f^{\prime}}=\frac{2 \pi}{\left(E_{g}^{f}-E_{g}^{f^{\prime}}\right)} \approx \frac{2 \pi}{\Delta M_{f f^{\prime}}^{g}}\left(\frac{v_{p}}{c}\right), \quad \text { for } \quad p \ll \frac{\left(m_{f}^{D}\right)^{2}}{4 m_{f}^{M}}, \quad\left(f \rightarrow f^{\prime}\right),
\end{array}
$$

where the second line (8.17) may be used for the case of cosmic neutrino background of temperature $\mathcal{O}\left(10^{-4}\right) \mathrm{eV}$.

These oscillations between the family flavors of gauged Majorana neutrinos have been important for experiments performed in ground and underground laboratories. Using eq. (8.14), neutrino Dirac masses $m_{f}^{D}$ (table 1 ) and experimental values [114]:

$$
\left|\Delta M_{21}^{g 2}\right| \approx 7.5 \times 10^{-5}(\mathrm{eV})^{2}, \quad\left|\Delta M_{31}^{g 2}\right| \approx 2.5 \times 10^{-3}(\mathrm{eV})^{2},
$$

neglecting the term $\left[\left(m_{1}^{D}\right)^{2} / 4 m_{1}^{M}\right]^{2}$ we obtain ratios $m_{3}^{D} / m_{3}^{M}=8.3 \times 10^{-10}$ and $m_{2}^{D} / m_{2}^{M}=$ $1.4 \times 10^{-8}$, and the Majorana masses $m_{3}^{M} \approx 2.9 \times 10^{8} \mathrm{GeV}$ and $m_{2}^{M} \approx 1.7 \times 10^{5} \mathrm{GeV}$. As a result, two sterile Majorana neutrino masses $(8.10) M_{3}^{s} \approx 2.9 \times 10^{8} \mathrm{GeV}$ and $M_{2}^{s} \approx$ $1.7 \times 10^{5} \mathrm{GeV}$, two gauged Majorana neutrino masses $(8.9) M_{3}^{g} \approx 5.0 \times 10^{-2} \mathrm{eV}$ and $M_{2}^{g} \approx$ $8.4 \times 10^{-3} \mathrm{eV}$, see table 2 . 
Among the three neutrino mass-squared differences (8.14), only two of them are independent for $\Delta M_{32}^{g 2}=\Delta M_{31}^{g 2}-\Delta M_{21}^{g 2} \approx \Delta M_{31}^{g 2}$. In principle we cannot determine the values $m_{1}^{M}, m_{1}^{D} / m_{1}^{M}$, and $\left[\left(m_{1}^{D}\right)^{2} / 4 m_{1}^{M}\right]^{2}$. However, we infer the hierarchy structure of Majorana masses $m_{2}^{M}$ and $m_{1}^{M}$

$$
\left(m_{2}^{M} / m_{1}^{M}\right) \approx\left(m_{3}^{M} / m_{2}^{M}\right) \approx 1.0 \times 10^{3}, \text { then } m_{1}^{M} \approx 1.7 \times 10^{2} \mathrm{GeV},
$$

on the basis of the reasons we discussed in the paragraph of eq. (3.19). This inference (8.19) leads to the ratio $m_{1}^{D} / m_{1}^{M} \approx 2.5 \times 10^{-8}$ and the lowest lying Majorana neutrino mass

$$
M_{1}^{g} \approx\left(m_{1}^{D}\right)^{2} / 4 m_{1}^{M} \approx 2.8 \times 10^{-5} \mathrm{eV} .
$$

Thus we tabulate the values (8.19) and (8.20) in the first column of table 2. These results satisfy the recent cosmological constrain [126] on the total mass of three light gauged neutrinos (8.9),

$$
\sum_{f=1,2,3} M_{f}^{g} \approx \sum_{f=1,2,3}\left(m_{f}^{D}\right)^{2} / 4 m_{f}^{M} \approx 5.8 \times 10^{-2} \mathrm{eV}<2.3 \times 10^{-1} \mathrm{eV} .
$$

Needless to say, it is important that the sensitivity of experiments and observations on neutrino masses can be reached at least to the level $\mathcal{O}\left(10^{-2}\right) \mathrm{eV}$.

\subsection{Flavor oscillations of sterile Majorana neutrinos}

We turn to discuss the family-flavor oscillations of three heavy sterile Majorana neutrinos (8.10). They are described by the mixing matrix $U_{R}^{\ell}=\mathcal{U}_{R}^{\nu \dagger} \mathcal{U}_{R}^{\ell}(3.18)$, the mass and mass-squared differences of sterile Majorana neutrino mass-eigenstates $(i, j=1,2,3)$, which are calculated by using eq. (8.8)

$$
\begin{aligned}
\Delta M_{f f^{\prime}}^{s} & \equiv M_{f}^{s}-M_{f^{\prime}}^{s} \approx m_{f}^{M}-m_{f^{\prime}}^{M} \\
\Delta M_{f f^{\prime}}^{s 2} & \equiv\left(M_{f}^{s}\right)^{2}-\left(M_{f^{\prime}}^{s}\right)^{2} \\
& \approx \frac{1}{2}\left(2 \Delta m_{f f^{\prime}}^{2 M}+\Delta m_{f f^{\prime}}^{2 D}\right) \approx \Delta m_{f f^{\prime}}^{2 M}
\end{aligned}
$$

Equation (8.22) is up to the order $\mathcal{O}\left\{\left(m_{i}^{D}\right)^{2} / 4 m_{i}^{M}\right\}$, eq. (8.23) is up to the order $\mathcal{O}\left\{\left[\left(m_{i}^{D}\right)^{2} / 4 m_{i}^{M}\right]^{2}\right\}$ and the definitions are

$$
\Delta m_{f f^{\prime}}^{2 M} \equiv\left(m_{f}^{M}\right)^{2}-\left(m_{f^{\prime}}^{M}\right)^{2}, \quad \Delta m_{f f^{\prime}}^{2 D} \equiv\left(m_{f}^{D}\right)^{2}-\left(m_{f^{\prime}}^{D}\right)^{2} .
$$

We $\Delta m_{f f^{\prime}}^{2 M} \gg \Delta m_{f f^{\prime}}^{2 D}$ and $\Delta m_{f f^{\prime}}^{M} \gg \Delta m_{f f^{\prime}}^{D}$, see table 2. The oscillating probability from the sterile flavor $\nu_{\alpha}^{s}$ to the sterile flavor $\nu_{\beta}^{s}$ reads

$$
P_{\nu_{\alpha}^{s} \longleftrightarrow \nu_{\beta}^{s}}(t)=\sum_{f f^{\prime}}\left(U_{R}^{\ell}\right)_{\alpha f}^{*}\left(U_{R}^{\ell}\right)_{\beta f}\left(U_{R}^{\ell}\right)_{\alpha f^{\prime}}\left(U_{R}^{\ell}\right)_{\beta f^{\prime}}^{*} \exp \left[-i\left(E_{s}^{f}-E_{s}^{f^{\prime}}\right) t\right] .
$$

The oscillating lengths of non-relativistic and relativistic sterile neutrinos are given by

$$
\begin{aligned}
& L_{s}^{f f^{\prime}}=\frac{2 \pi}{\left(E_{s}^{f}-E_{s}^{f^{\prime}}\right)} \approx \frac{2 \pi}{\Delta M_{f f^{\prime}}^{s}}\left(\frac{v_{p}}{c}\right), \text { for } \quad m_{f}^{D} \ll p \ll m_{f}^{M}, \quad\left(f \rightarrow f^{\prime}\right) \\
& L_{s}^{f f^{\prime}}=\frac{2 \pi}{\left(E_{s}^{f}-E_{s}^{f^{\prime}}\right)} \approx \frac{2 \pi(2 p)}{\Delta M_{f f^{\prime}}^{s 2}}, \quad \text { for } \quad m_{f}^{D} \ll m_{f}^{M}<p, \quad\left(f \rightarrow f^{\prime}\right) .
\end{aligned}
$$


Table 2 shows the large mass and mass-squared differences (8.22) and (8.23), therefore in addition to their sterility the oscillating lengths between the flavors of sterile Majorana neutrinos are too small to be relevant for experiments in ground and underground laboratories. However these oscillations could be relevant in early universe evolution, depending on the Majorana masses $m^{M}$ or the energy scale $v_{\text {sterile }}$ of the lepton-number violation.

\subsection{Oscillations between gauged and sterile Majorana neutrinos}

Following eqs. (8.7)-(8.11), the oscillating probability between two mass eigenstates of gauged Majorana neutrino $\nu_{g}^{f}$ and sterile Majorana neutrino $\nu_{s}^{f}$ reads

$$
P_{\nu_{g}^{f} \longleftrightarrow \nu_{s}^{f}}(t)=1-2^{-1} \sin ^{2} 2 \theta_{f}\left[1-\cos \left(E_{g}^{f}-E_{s}^{f}\right) t\right],
$$

where $f=1,2,3$ and

$$
\begin{array}{lll}
\left(E_{s}^{f}-E_{g}^{f}\right) \approx m_{f}^{M}, & & \left(m_{f}^{D}\right)^{2} / 4 m_{f}^{M} \ll p \ll m_{f}^{M}, \\
\left(E_{s}^{f}-E_{g}^{f}\right) \approx\left(m_{f}^{M}\right)^{2} /(2 p), & & \left(m_{f}^{D}\right)^{2} / 4 m_{f}^{M} \ll m_{f}^{M}<p .
\end{array}
$$

for non-relativstic and relativistic cases. The oscillating lengths read

$$
\begin{array}{ll}
L_{s g}^{f}=\frac{2 \pi}{\left(E_{s}^{f}-E_{g}^{f}\right)} \approx \frac{2 \pi}{m_{f}^{M}}\left(\frac{v_{p}}{c}\right), & \text { for } \quad \frac{\left(m_{f}^{D}\right)^{2}}{4 m_{f}^{M}} \ll p \ll m_{f}^{M}, \\
L_{s g}^{f}=\frac{2 \pi}{\left(E_{s}^{f}-E_{g}^{f}\right)} \approx \frac{2 \pi(2 p)}{\left(m_{f}^{M}\right)^{2}}, \quad \text { for } \quad \frac{\left(m_{f}^{D}\right)^{2}}{4 m_{f}^{M}} \ll m_{f}^{M}<p .
\end{array}
$$

The large values of Majorana mass $m_{f}^{M}$ and mass-squared $\left(m_{f}^{M}\right)^{2}$ [see table 2] show the small oscillating lengths. The small mixing angle (8.11) indicates the small oscillating probabilities (8.28) between gauged and sterile Majorana neutrinos.

The oscillating probability between the sterile flavor $\nu_{\alpha}^{s}$ and the gauged flavor $\nu_{\beta}^{g}$ reads

$$
P_{\nu_{\alpha}^{s} \longleftrightarrow \nu_{\beta}^{g}}(t)=\sum_{f f^{\prime}}\left(U_{R}^{\ell}\right)_{\alpha f}^{*}\left(U_{L}^{\ell}\right)_{\beta f}\left(U_{R}^{\ell}\right)_{\alpha f^{\prime}}\left(U_{L}^{\ell}\right)_{\beta f^{\prime}}^{*} \exp \left[-i\left(E_{s}^{f}-E_{g}^{f^{\prime}}\right) t\right] .
$$

Apart from mixing matrices, via the oscillatory factor $\exp \left[-i\left(E_{s}^{f}-E_{g}^{f^{\prime}}\right) t\right]$ the oscillation probability depends on the sum over the mass differences $\Delta m_{f f^{\prime}}$ or mass-squared differences $\Delta m_{f f^{\prime}}^{2}$ of mass-eigenstates $\left(f \neq f^{\prime}\right)$ of two flavor neutrinos $\nu_{\alpha}^{s}$ and $\nu_{\beta}^{g}$. Given neutrino energies, and their masses or mass-squared differences, one can select an oscillating length $L_{f f^{\prime}}$ that is relevant for a possible observation or effect. The mass spectra (table 2) of gauged and sterile neutrinos show a large difference of their mass scales, indicating oscillations between them at short distances. For the example $M_{1}^{s}$ and $M_{1}^{g}$ cases, the oscillating length is at least $10^{-1} \mathrm{GeV}^{-1}\left(v_{p} / c\right)$ for $p \ll 10^{2} \mathrm{GeV}$ or $10^{-1} \mathrm{GeV}^{-1}\left(p / 10^{2} \mathrm{GeV}\right)$ for $p \gg 10^{2} \mathrm{GeV}$, as shown in eqs. (8.31) and (8.32). The latter implies the possibility (8.33) for very high-energy electron neutrinos converting themselves into sterile neutrinos. It seems to be hard to detect the oscillations between gauged and sterile Majorana neutrinos in experiments performed in space, ground and underground laboratories. However these 
oscillations could be important in early universe evolution, depending on the energy scale $v_{\text {sterile }}$ or $m^{M}$ of the lepton-number violation.

Actually, the probabilities of three flavor oscillations (8.15), (8.25) and (8.33) are described by the following $6 \times 6$ mixing matrix, see eq. (3.17)

$$
\frac{1}{\sqrt{2}}\left(\begin{array}{cc}
U_{L}^{\ell} & e^{i \varphi_{1}} \mathcal{U}_{L}^{\nu \dagger} \mathcal{U}_{R}^{\ell} \\
e^{i \varphi_{2}} \mathcal{U}_{R}^{\nu \dagger} \mathcal{U}_{L}^{\ell} & U_{R}^{\ell}
\end{array}\right),
$$

where $e^{i \varphi_{1}}$ is a relative phase between $\mathcal{U}_{L}^{\nu \dagger}$ and $\mathcal{U}_{R}^{\ell}$, and $e^{i \varphi_{2}}$ is another relative phase between $\mathcal{U}_{R}^{\nu \dagger}$ and $\mathcal{U}_{L}^{\ell}$. The maxing matrix (8.34) is unitary, if $\varphi_{2}-\varphi_{1}=n \pi, \quad n=1,2,3, \cdots$. The diagonal parts $U_{L}^{\ell}$ (PMNS) and $U_{R}^{\ell}$ respectively represent the mixing matrices for the gauged flavor oscillations (8.15) and sterile flavor oscillations (8.25), and the off-diagonal parts represent the mixing matrices for the gauged-sterile flavor oscillations (8.33).

\section{$9 \quad$ A summary and some remarks}

We end this lengthy article by making some relevant remarks and preliminary discussions on possible consequences of SM gauged particle, Majorana sterile and gauged neutrino spectra, tables 1 and 2 qualitatively obtained in this article.

\subsection{SM fermion Dirac masses and Yukawa couplings}

Due to the ground-state (vacuum) alignment of the effective theory of relevant four-fermion operators, the top-quark mass is generated by the SSB, and other fermion masses are originated from the ESB terms, which are induced by the top-quark mass via the fermion-family mixing, quark-lepton interactions and vector-like $W^{ \pm}$-boson coupling at high energies. As a consequence, the fermion masses are functions of the top-quark mass and the fermion Yukawa couplings are functions of the top-quark Yukawa coupling. Based on the approach adopted and the results obtained in ref. [97], we study the inhomogeneous SD-equations for all SM fermion masses with the ESB terms and obtain the hierarchy patter of fermion masses and Yukawa couplings, consistently with the hierarchy patter of the fermion-family mixing matrix elements. However, we do not discuss the detailed properties of the quarkflavor mixing matrices (3.8), the lepton-flavor mixing matrices (3.17) [or (8.34)], where the CKM matrix $U^{q}=\mathcal{U}_{L}^{u \dagger} \mathcal{U}_{L}^{d}$ and PMNS matrix $U_{L}^{\ell}=\mathcal{U}_{L}^{\nu^{\dagger}} \mathcal{U}_{L}^{\ell}$ are particular examples relating to the coupling vertex of $W^{ \pm}$-bosons. Also we do not discuss the quark-lepton flavor mixing matrices (3.22) relates to the quark-lepton interactions. These unitary matrices are composed by the eigenvectors corresponding to eigenvalues (fermion-mass spectra) of fermion-mass matrices. They code all information about mixing angles and CP-violations.

Relating to the slowly varying Yukawa coupling $\bar{g}_{t}(\mu)$ of the top quark, see section 4.2.2, all fermion Yukawa couplings obtained slowly vary from $1 \mathrm{GeV}$ to $13.5 \mathrm{TeV}$. These features imply that it should be hard to have any detectable nonresonant signatures in the LHC ppcollisions, showing the deviations from the SM with the elementary Higgs boson. All these results are preliminarily qualitative, and they should receive the high-order corrections and some non-perturbative contributions. It should be emphasized that these qualitative 
results cannot be quantitatively compared with the SM precision tests. The quantitative study is a difficult and challenging task and one will probably be able to carry on it by using a numerical approach in future. Nevertheless, these qualitative results may give us some insight into the long-standing problem of fermion-mass origin and hierarchy.

\subsection{Neutrinos and dark-matter particles}

The values of three light gauged Majorana neutrino masses $M_{f}^{g}$ give some insight into the neutrino problems that directly relate to the absolute values of neutrino masses. The $\beta$-decay rate depends on $m_{\beta}^{2}=\sum_{f}\left|U_{e f}^{\ell}\right|^{2}\left(M_{f}^{g}\right)^{2}$. The double $\beta$-decay rate depends on $m_{2 \beta}=\sum_{f}\left|U_{e f}^{\ell}\right|^{2} M_{f}^{g} \tilde{\eta}_{f}$, where $\tilde{\eta}_{f}= \pm 1$ is the CP eigenvalue of the mass eigenstate $\nu_{f}^{g}$ (8.9). The $M_{3}^{g}$ and $M_{2}^{g}$ values, as well as $M_{1}^{g}$ range in table 2 seem to be in agreement with the analysis of using experimental data of mass-squared differences (8.18) and the PMNS mixing matrix $U_{L}^{\ell}$ in the normal hierarchy case (see for example ref. [128]). In addition to the measurements of neutrino mixing angles, it is obviously important to experimentally measure neutrino masses with a sensitivity below $10^{-2} \mathrm{eV}$ so as to determine the neutrino features.

The very massive sterile neutrinos (8.10) of Majorana type, whose masses $M_{1}^{s} \sim$ $10^{2} \mathrm{GeV}, M_{2}^{s} \sim 10^{5} \mathrm{GeV}$ and $M_{3}^{s} \sim 10^{8} \mathrm{GeV}$ (see table 2 ), could be candidates for very massive cold dark-matter $(\mathrm{DM})$ particles. While, the right-handed sterile neutrinos $\nu_{R}^{f}$ of Dirac type, whose Dirac masses $M_{1}^{D} \sim 10^{-6} \mathrm{GeV}, M_{2}^{D} \sim 10^{-3} \mathrm{GeV}$ and $M_{3}^{D} \sim 10^{-1} \mathrm{GeV}$ (see table 2), could be considered as light, weak-interacting "warm" DM particles, in particular the one $\nu_{R}^{1}$ with a few $\mathrm{KeV}$ mass. Moreover, the sterile composite scalar particle (8.4) could be probably a candidate for a massive cold DM particle, though we do not know its mass $m_{H}^{M}$ (8.5), i.e., the scale of lepton-number non-conservation. What is then the candidate for light, non-interacting warm DM particle? We expect that it should be the pseudoscalar boson $\phi^{M}$ (8.3), which acquires a small mass $m_{\phi}$ by the analogy of the PCAC (partially conserved axial-vector current) and soft pion theorems,

$$
\partial^{\mu} A_{\mu}=f_{\phi} m_{\phi}^{2} \phi^{M}, \quad A_{\mu}=\sum_{f} \bar{\nu}_{R}^{f c} \gamma^{\mu} \gamma_{5} \nu_{R}^{f}
$$

This is due to the presence of soft explicit $U_{\text {lepton }}(1)$-symmetry breaking terms $\tilde{m}_{f}^{s} \bar{\nu}_{R}^{f c} \nu_{R}^{f}$ $\left(\tilde{m}_{f}^{s} \ll m_{f}^{M}\right)$ in eq. (8.6). The $f_{\phi}$ is the pseudoscalar boson $\phi^{M}$ decay constant relating to the processes $\phi^{M} \rightarrow \nu_{R}^{f c}+\nu_{R}^{f}$. Both mass $m_{\phi}$ and decay constant $f_{\phi}$ depend on the soft explicit breaking scale $\tilde{m}_{f}^{s}$ of $U_{\text {lepton}}(1)$-symmetry. It is worthwhile to notice that both sterile Majorana neutrinos (the candidates of cold DM particles) and the sterile pseudoscalar boson (the candidate of warm DM particle) carry two units of lepton number. This implies that the relevant processes of these sterile particles interacting with the SM particles, though very weak, should violate the lepton-number conservation and lead to the asymmetry of matter and anti-matter. At the end we mention that for strong coupling $G$ the relevant four-fermion operators (2.12) and (3.9) present the interactions of DM and SM particles, and form gauged and neutral composite particles as resonances of masses at $\mathrm{TeV}$ scale, then these composite particles (resonances) decay into their constitutes - SM and/or DM particles [89, 94, 97, 107, 130]. 


\section{Acknowledgments}

The author thanks Prof. Hagen Kleinert for discussions on the IR- and UV-stable fixed points of quantum field theories, Prof. Remo Ruffini for discussions on the Einstein gravitational theory and Prof. Zhiqing Zhang for discussions on the LHC physics. The author also thanks the anonymous referee for his/her effort of reviewing this lengthy article.

Open Access. This article is distributed under the terms of the Creative Commons Attribution License (CC-BY 4.0), which permits any use, distribution and reproduction in any medium, provided the original author(s) and source are credited.

\section{References}

[1] Y. Nambu and G. Jona-Lasinio, Dynamical model of elementary particles based on an analogy with superconductivity. 1, Phys. Rev. 122 (1961) 345 [INSPIRE].

[2] F. Englert and R. Brout, Broken symmetry and the mass of gauge vector mesons, Phys. Rev. Lett. 13 (1964) 321 [INSPIRE].

[3] P.W. Higgs, Broken symmetries, massless particles and gauge fields, Phys. Lett. 12 (1964) 132 [INSPIRE].

[4] P.W. Higgs, Broken symmetries and the masses of gauge bosons, Phys. Rev. Lett. 13 (1964) 508 [INSPIRE].

[5] P.W. Higgs, Spontaneous symmetry breakdown without massless bosons, Phys. Rev. 145 (1966) 1156 [INSPIRE].

[6] G.S. Guralnik, C.R. Hagen and T.W.B. Kibble, Global conservation laws and massless particles, Phys. Rev. Lett. 13 (1964) 585 [INSPIRE].

[7] T.W.B. Kibble, Symmetry breaking in non-abelian gauge theories, Phys. Rev. 155 (1967) 1554 [INSPIRE].

[8] ATLAS collaboration, Observation of a new particle in the search for the standard model Higgs boson with the ATLAS detector at the LHC, Phys. Lett. B 716 (2012) 1 [arXiv:1207.7214] [INSPIRE].

[9] CMS collaboration, Observation of a new boson at a mass of $125 \mathrm{GeV}$ with the CMS experiment at the LHC, Phys. Lett. B 716 (2012) 30 [arXiv:1207.7235] [InSPIRE].

[10] CDF collaboration, F. Abe et al., Observation of top quark production in $\bar{p} p$ collisions, Phys. Rev. Lett. 74 (1995) 2626 [hep-ex/9503002] [INSPIRE].

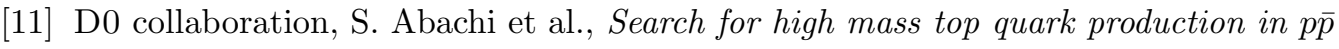
collisions at $\sqrt{s}=1.8$ TeV, Phys. Rev. Lett. 74 (1995) 2422 [hep-ex/9411001] [INSPIRE].

[12] R. Gatto, G. Sartori and M. Tonin, Weak selfmasses, Cabibbo angle and broken $\mathrm{SU}(2) \times \mathrm{SU}(2)$, Phys. Lett. B 28 (1968) 128 [INSPIRE].

[13] N. Cabibbo and L. Maiani, Dynamical interrelation of weak, electromagnetic and strong interactions and the value of theta, Phys. Lett. B 28 (1968) 131 [INSPIRE].

[14] K. Tanaka and P. Tarjanne, Cabibbo angle and selfconsistency condition, Phys. Rev. Lett. 23 (1969) 1137 [INSPIRE]. 
[15] R.J. Oakes, $\mathrm{SU}(2) \times \mathrm{SU}(2)$ breaking and the Cabibbo angle, Phys. Lett. B 29 (1969) 683 [INSPIRE].

[16] H. Genz, J. Katz, L.R. Ram Mohan and S. Tatur, Chiral symmetry breaking and the cabibbo angle, Phys. Rev. D 6 (1972) 3259 [InSPIRE].

[17] H. Pagels, Vacuum stability and the Cabibbo angle, Phys. Rev. D 11 (1975) 1213 [InSPIRE].

[18] A. Ebrahim, Chiral $\mathrm{SU}(4) \times \mathrm{SU}(4)$ symmetry breaking and the Cabibbo angle, Phys. Lett. $\mathrm{B}$ 69 (1977) 229 [INSPIRE].

[19] H. Fritzsch, Calculating the Cabibbo angle, Phys. Lett. B 70 (1977) 436 [InSPIRE].

[20] S. Weinberg, The problem of mass, Trans. New York Acad. Sci. 38 (1977) 185.

[21] A. De Rujula, H. Georgi and S.L. Glashow, A theory of flavor mixing, Annals Phys. 109 (1977) 258 [INSPIRE].

[22] F. Wilczek and A. Zee, Discrete flavor symmetries and a formula for the Cabibbo angle, Phys. Lett. B 70 (1977) 418 [Erratum ibid. B 72 (1978) 504] [InSPIRE].

[23] R.N. Mohapatra and G. Senjanović, Cabibbo Angle, CP-violation and quark masses, Phys. Lett. B 73 (1978) 176 [INSPIRE].

[24] F. Wilczek and A. Zee, Horizontal interaction and weak mixing angles, Phys. Rev. Lett. 42 (1979) 421 [INSPIRE].

[25] D. Wyler, The Cabibbo angle in the $\mathrm{SU}(2)_{L} \times \mathrm{U}(1)$ gauge theories, Phys. Rev. D 19 (1979) 330 [INSPIRE].

[26] H. Fritzsch, Hierarchical chiral symmetries and the quark mass matrix, Phys. Lett. B 184 (1987) 391 [INSPIRE].

[27] P.H. Frampton and Y. Okada, Simplified symmetric quark mass matrices and flavor mixing, Mod. Phys. Lett. A 6 (1991) 2169 [inSPIRE].

[28] J.L. Rosner and M.P. Worah, Models of the quark mixing matrix, Phys. Rev. D 46 (1992) 1131 [INSPIRE].

[29] S. Raby, Introduction to theories of fermion masses, hep-ph/9501349 [INSPIRE].

[30] T. Ito and M. Tanimoto, The Unitarity triangle and quark mass matrices on the NNI basis, Phys. Rev. D 55 (1997) 1509 [hep-ph/9603393] [INSPIRE].

[31] Z.-Z. Xing, Implications of the quark mass hierarchy on flavor mixings, J. Phys. G 23 (1997) 1563 [hep-ph/9609204] [INSPIRE].

[32] S.-S. Xue, Quark masses and mixing angles, Phys. Lett. B 398 (1997) 177 [hep-ph/9610508] [INSPIRE].

[33] S.-S. Xue, Neutrino masses and mixings, Mod. Phys. Lett. A 14 (1999) 2701 [hep-ph/9706301] [INSPIRE].

[34] R. Barbieri, L.J. Hall, S. Raby and A. Romanino, Unified theories with U(2) flavor symmetry, Nucl. Phys. B 493 (1997) 3 [hep-ph/9610449] [INSPIRE].

[35] D. Falcone and F. Tramontano, Relation between quark masses and weak mixings, Phys. Rev. D 59 (1999) 017302 [hep-ph/9806496] [INSPIRE].

[36] A. Mondragon and E. Rodriguez-Jauregui, The breaking of the flavor permutational symmetry: mass textures and the CKM matrix, Phys. Rev. D 59 (1999) 093009 [hep-ph/9807214] [INSPIRE]. 
[37] A. Mondragon and E. Rodriguez-Jauregui, The CP-violating phase $\delta_{13}$ and the quark mixing angles $\theta_{13}, \theta_{23}$ and $\theta_{12}$ from flavor permutational symmetry breaking, Phys. Rev. D 61 (2000) 113002 [hep-ph/9906429] [INSPIRE].

[38] H. Fritzsch and Z.-Z. Xing, Mass and flavor mixing schemes of quarks and leptons, Prog. Part. Nucl. Phys. 45 (2000) 1 [hep-ph/9912358] [INSPIRE].

[39] G.C. Branco, D. Emmanuel-Costa and C. Simoes, Nearest-neighbour interaction from an abelian symmetry and deviations from hermiticity, Phys. Lett. B 690 (2010) 62 [arXiv: 1001.5065] [INSPIRE].

[40] F. González Canales et al., Quark sector of S3 models: classification and comparison with experimental data, Phys. Rev. D 88 (2013) 096004 [arXiv:1304.6644] [INSPIRE].

[41] L.J. Hall and A. Rasin, On the generality of certain predictions for quark mixing, Phys. Lett. B 315 (1993) 164 [hep-ph/9303303] [INSPIRE].

[42] A. Rasin, Diagonalization of quark mass matrices and the Cabibbo-Kobayashi-Maskawa matrix, hep-ph/9708216 [INSPIRE].

[43] A. Rasin, Hierarchical quark mass matrices, Phys. Rev. D 58 (1998) 096012 [hep-ph/9802356] [INSPIRE].

[44] H. Fritzsch and Z.-Z. Xing, The light quark sector, CP-violation and the unitarity triangle, Nucl. Phys. B 556 (1999) 49 [hep-ph/9904286] [inSPIRE].

[45] Z.-Z. Xing, Model-independent access to the structure of quark flavor mixing, Phys. Rev. D 86 (2012) 113006 [arXiv:1211.3890] [INSPIRE].

[46] H. Ishimori, T. Kobayashi, H. Ohki, Y. Shimizu, H. Okada and M. Tanimoto, Non-abelian discrete symmetries in particle physics, Prog. Theor. Phys. Suppl. 183 (2010) 1 [arXiv: 1003.3552] [INSPIRE].

[47] S.F. King and C. Luhn, Neutrino mass and mixing with discrete symmetry, Rept. Prog. Phys. 76 (2013) 056201 [arXiv: 1301.1340] [INSPIRE].

[48] J. Schechter and J.W.F. Valle, Neutrino masses in $\mathrm{SU}(2) \times \mathrm{U}(1)$ theories, Phys. Rev. D 22 (1980) 2227 [INSPIRE].

[49] H. Fritzsch, Quark masses and flavor mixing, Nucl. Phys. B 155 (1979) 189 [InSPIRE].

[50] A.E. Faraggi, String phenomenology: past, present and future perspectives, Galaxies 2 (2014) 223 [arXiv: 1404.5180] [inSPIRE].

[51] M. Dine, Supersymmetry and string theory: beyond the standard model, Cambridge University Press, Cambridge U.K. (2015).

[52] H. Fritzsch, Weak interaction mixing in the six-quark theory, Phys. Lett. B 73 (1978) 317 [INSPIRE].

[53] P. Ramond, R.G. Roberts and G.G. Ross, Stitching the Yukawa quilt, Nucl. Phys. B 406 (1993) 19 [hep-ph/9303320] [INSPIRE].

[54] G.C. Branco, D. Emmanuel-Costa and R. Gonzalez Felipe, Texture zeros and weak basis transformations, Phys. Lett. B 477 (2000) 147 [hep-ph/9911418] [INSPIRE].

[55] R.G. Roberts, A. Romanino, G.G. Ross and L. Velasco-Sevilla, Precision test of a fermion mass texture, Nucl. Phys. B 615 (2001) 358 [hep-ph/0104088] [inSPIRE].

[56] H. Fritzsch and Z.-Z. Xing, Four zero texture of Hermitian quark mass matrices and current experimental tests, Phys. Lett. B 555 (2003) 63 [hep-ph/0212195] [INSPIRE]. 
[57] M. Gupta and G. Ahuja, Flavor mixings and textures of the fermion mass matrices, Int. J. Mod. Phys. A 27 (2012) 1230033 [arXiv: 1302.4823] [INSPIRE].

[58] S. Pakvasa and H. Sugawara, CP violation in six quark model, Phys. Rev. D 14 (1976) 305 [INSPIRE].

[59] S. Pakvasa and H. Sugawara, Discrete symmetry and Cabibbo angle, Phys. Lett. B 73 (1978) 61 [INSPIRE].

[60] E. Derman, Flavor unification, $\tau$ decay and $b$ decay within the six quark six lepton Weinberg-Salam model, Phys. Rev. D 19 (1979) 317 [INSPIRE].

[61] Y. Yamanaka, H. Sugawara and S. Pakvasa, Permutation symmetries and the fermion mass matrix, Phys. Rev. D 25 (1982) 1895 [Erratum ibid. D 29 (1984) 2135] [InSPIRE].

[62] R. Yahalom, Horizontal permutation symmetry, fermion masses and pseudogoldstone bosons in $\mathrm{SU}(2)_{L} \times \mathrm{U}(1)$, Phys. Rev. D 29 (1984) 536 [INSPIRE].

[63] G. Altarelli, Status of neutrino mass and mixing, Int. J. Mod. Phys. A 29 (2014) 1444002 [arXiv: 1404.3859] [INSPIRE].

[64] S.F. King, A. Merle, S. Morisi, Y. Shimizu and M. Tanimoto, Neutrino mass and mixing: from theory to experiment, New J. Phys. 16 (2014) 045018 [arXiv:1402.4271] [InSPIRE].

[65] H. Fritzsch and Z.-Z. Xing, Lepton mass hierarchy and neutrino mixing, Phys. Lett. B 634 (2006) 514 [hep-ph/0601104] [INSPIRE].

[66] H. Fritzsch and Z.-Z. Xing, Relating the neutrino mixing angles to a lepton mass hierarchy, Phys. Lett. B 682 (2009) 220 [arXiv:0911.1857] [INSPIRE].

[67] W.G. Hollik and U.J. Saldaña Salazar, The double mass hierarchy pattern: simultaneously understanding quark and lepton mixing, Nucl. Phys. B 892 (2015) 364 [arXiv:1411.3549] [INSPIRE].

[68] G. Lazarides, Q. Shafi and C. Wetterich, Proton lifetime and fermion masses in an $\mathrm{SO}(10)$ model, Nucl. Phys. B 181287 [InSPIRE].

[69] G. Altarelli and G. Blankenburg, Different $\mathrm{SO}(10)$ paths to fermion masses and mixings, JHEP 03 (2011) 133 [arXiv:1012.2697] [INSPIRE].

[70] A.S. Joshipura and K.M. Patel, Fermion masses in SO(10) models, Phys. Rev. D 83 (2011) 095002 [arXiv: 1102.5148] [INSPIRE].

[71] W. Grimus and H. Kühböck, Fermion masses and mixings in a renormalizable $\mathrm{SO}(10) \times Z_{2}$ GUT, Phys. Lett. B 643 (2006) 182 [hep-ph/0607197] [INSPIRE].

[72] L. Lavoura, H. Kuhbock and W. Grimus, Charged-fermion masses in $\mathrm{SO}(10)$ : analysis with scalars in $10+120$, Nucl. Phys. B 754 (2006) 1 [hep-ph/0603259] [INSPIRE].

[73] C.H. Albright and S. Nandi, New approach for the construction of fermion mass matrices in SO(10) SUSY GUTs, Phys. Rev. Lett. 73 (1994) 930 [hep-ph/9311227] [INSPIRE].

[74] C.H. Albright and S.M. Barr, Fermion masses in $\mathrm{SO}(10)$ with a single adjoint Higgs field, Phys. Rev. D 58 (1998) 013002 [hep-ph/9712488] [INSPIRE].

[75] C.T. Hill, Topcolor: top quark condensation in a gauge extension of the standard model, Phys. Lett. B 266 (1991) 419 [INSPIRE].

[76] C.T. Hill, Topcolor assisted technicolor, Phys. Lett. B 345 (1995) 483 [hep-ph/9411426] [INSPIRE]. 
[77] C.T. Hill, 'Super'-dilatation symmetry of the top-Higgs system, Phys. Rev. D 87 (2013) 065002 [arXiv: 1211.2773] [INSPIRE].

[78] C.T. Hill, Quark and lepton masses from renormalization group fixed points, Phys. Rev. D 24 (1981) 691 [INSPIRE].

[79] C.T. Hill, C.N. Leung and S. Rao, Renormalization group fixed points and the Higgs boson spectrum, Nucl. Phys. B 262 (1985) 517 [INSPIRE].

[80] J. Bagger, S. Dimopoulous and E. Masso, Renormalization-group constraints in supersymmetric theories, Phys. Rev. Lett. 55 (1985) 920.

[81] W.A. Bardeen, C.T. Hill and M. Lindner, Minimal dynamical symmetry breaking of the standard model, Phys. Rev. D 41 (1990) 1647 [INSPIRE].

[82] Y. Nambu, in Proceedings of the 1989 workshop on Dynamical Symmetry Breaking, T. Muta and K. Yamawaki eds., Nagoya University, Nagoya, Japan (1990).

[83] V.A. Miransky, M. Tanabashi and K. Yamawaki, Is the $t$ quark responsible for the mass of $W$ and $Z$ bosons?, Mod. Phys. Lett. A 4 (1989) 1043 [inSPIRE].

[84] H. Kleinert, Particles and quantum field, World Scientific, Singapore (2016), http://klnrt.de/b6.

[85] W.J. Marciano, Heavy top-quark mass predictions, Phys. Rev. Lett. 62 (1989) 2793.

[86] G. Cvetič, Top quark condensation, Rev. Mod. Phys. 71 (1999) 513 [hep-ph/9702381] [INSPIRE].

[87] C.T. Hill and E.H. Simmons, Strong dynamics and electroweak symmetry breaking, Phys. Rept. 381 (2003) 235 [Erratum ibid. 390 (2004) 553] [hep-ph/0203079] [INSPIRE].

[88] S.-S. Xue, Higgs boson and top-quark masses and parity-symmetry restoration, Phys. Lett. B 727 (2013) 308 [arXiv: 1308.6486] [INSPIRE].

[89] S.-S. Xue, Ultraviolet fixed point and massive composite particles in TeV scales, Phys. Lett. B 737 (2014) 172 [arXiv:1405.1867] [INSPIRE].

[90] S.-S. Xue, A lattice chiral theory with multi-fermion couplings, Phys. Lett. B 381 (1996) 277 [hep-lat/9605003] [INSPIRE].

[91] S.-S. Xue, A possible scaling region of chiral fermions on a lattice, Nucl. Phys. B 486 (1997) 282 [hep-lat/9605005] [INSPIRE].

[92] S.-S. Xue, Chiral gauged fermions on a lattice, Nucl. Phys. B 580 (2000) 365 [hep-lat/0002026] [INSPIRE].

[93] S.-S. Xue, A Further study of the possible scaling region of lattice chiral fermions, Phys. Rev. D 61 (2000) 054502 [hep-lat/9910013] [InSPIRE].

[94] S.-S. Xue, On the standard model and parity conservation, J. Phys. G 29 (2003) 2381.

[95] S.-S. Xue, Do high-energy neutrinos travel faster than photons in a discrete space-time?, Phys. Lett. B 706 (2011) 213 [arXiv:1110.1317] [InSPIRE].

[96] K. Cheung, W.-Y. Keung and T.-C. Yuan, Top quark forward-backward asymmetry, Phys. Lett. B 682 (2009) 287 [arXiv:0908.2589] [INSPIRE].

[97] S.-S. Xue, Vectorlike $W^{ \pm}$-boson coupling at TeV and third family fermion masses, Phys. Rev. D 93 (2016) 073001 [arXiv: 1506.05994] [INSPIRE]. 
[98] S.-S. Xue, Detailed discussions and calculations of quantum Regge calculus of Einstein-Cartan theory, Phys. Rev. D 82 (2010) 064039 [arXiv:0912.2435] [InSPIRE].

[99] S.-S. Xue, Quantum Regge calculus of Einstein-Cartan theory, Phys. Lett. B 682 (2009) 300 [arXiv:0902.3407] [INSPIRE].

[100] S.-S. Xue, The phase and critical point of quantum Einstein-Cartan gravity, Phys. Lett. B 711 (2012) 404 [arXiv:1112.1323] [INSPIRE].

[101] H.B. Nielsen and M. Ninomiya, Absence of neutrinos on a lattice. 1. Proof by homotopy theory, Nucl. Phys. B 185 (1981) 20 [Erratum ibid. B 195 (1982) 541] [InSPIRE].

[102] H.B. Nielsen and M. Ninomiya, Absence of Neutrinos on a Lattice. 2. Intuitive Topological Proof, Nucl. Phys. B 193 (1981) 173 [INSPIRE].

[103] H.B. Nielsen and M. Ninomiya, No go theorem for regularizing chiral fermions, Phys. Lett. 105B (1981) 219 [INSPIRE].

[104] H.B. Nielsen and M. Ninomiya, Intuitive understanding of anomalies: A Paradox with regularization, Int. J. Mod. Phys. A 6 (1991) 2913 [INSPIRE].

[105] C. Itzykson and J.B. Zuber, Quantum field theory, McGraw-Hill Inc., U.S.A. (2006).

[106] H. Kleinert, On the hadronization of quark theories, in Understanding the fundamental constituents of matter, A. Zichichi ed., Plenum Press, New York U.S.A. (1978).

[107] S.-S. Xue, Resonant and nonresonant new phenomena of four-fermion operators for experimental searches, Phys. Lett. B 744 (2015) 88 [arXiv:1501.06844] [INSPIRE].

[108] http://atlas.ch/.

[109] S.-S. Xue, Why is the top quark much heavier than other fermions?, Phys. Lett. B 721 (2013) 347 [arXiv: 1301.4254] [inSPIRE].

[110] E. Eichten and J. Preskill, Chiral gauge theories on the lattice, Nucl. Phys. B 268 (1986) 179 [INSPIRE].

[111] M. Creutz, M. Tytgat, C. Rebbi and S.-S. Xue, Lattice formulation of the standard model, Phys. Lett. B 402 (1997) 341 [hep-lat/9612017] [INSPIRE].

[112] T.P. Cheng and L.F. Li, Gauge theory of elementary particle physics, Oxford University Press, Oxford U.K. (1984).

[113] R.N. Mohapatra, P.B. Pal, Massive neutrinos in physics and astro-physics, $3^{\text {rd }}$ edition, World Scientific, Singapore (2004).

[114] Particle Data Group collaboration, J. Beringer et al., Review of particle physics, Phys. Rev. D 86 (2012) 010001 [INSPIRE].

[115] M.C. Gonzalez-Garcia, M. Maltoni and T. Schwetz, Updated fit to three neutrino mixing: status of leptonic CP-violation, JHEP 11 (2014) 052 [arXiv:1409.5439] [INSPIRE].

[116] CDF collaboration, T. Aaltonen et al., Forward-backward asymmetry in top quark production in p $\bar{p}$ collisions at sqrts $=1.96$ TeV, Phys. Rev. Lett. 101 (2008) 202001 [arXiv: 0806.2472] [INSPIRE].

[117] D0 collaboration, V.M. Abazov et al., First measurement of the forward-backward charge asymmetry in top quark pair production, Phys. Rev. Lett. 100 (2008) 142002 [arXiv: 0712.0851] [INSPIRE]. 
[118] R. Fukuda and T. Kugo, Schwinger-Dyson Equation for Massless Vector Theory and Absence of Fermion Pole, Nucl. Phys. B 117 (1976) 250 [InSPIRE].

[119] C.N. Leung, S.T. Love and W.A. Bardeen, Spontaneous Symmetry Breaking in Scale Invariant Quantum Electrodynamics, Nucl. Phys. B 273 (1986) 649 [INSPIRE].

[120] C.N. Leung, S.T. Love and W.A. Bardeen, Aspects of dynamical symmetry breaking in gauge field theories, Nucl. Phys. B 323 (1989) 493 [INSPIRE].

[121] A. Kocić, S. Hands, J.B. Kogut and E. Dagotto, The equation of state and critical exponents in quenched strongly coupled QED, Nucl. Phys. B 347 (1990) 217 [INSPIRE].

[122] G. Preparata and S.-S. Xue, Do we live on a lattice? Fermion masses from Planck mass, Phys. Lett. B 264 (1991) 35 [InSPIRE].

[123] G. Preparata and S.-S. Xue, A possible massive solution to Dyson equation with small gauge coupling, Phys. Lett. B 302 (1993) 442 [INSPIRE].

[124] G. Preparata and S.-S. Xue, Mass relation between top and bottom quarks, Phys. Lett. B 325 (1994) 161 [hep-lat/9401017] [INSPIRE].

[125] S.-S. Xue, Fine-tuning the low-energy physics, Mod. Phys. Lett. A 15 (2000) 1089.

[126] Planck collaboration, P.A.R. Ade et al., Planck 2015 results. XIII. Cosmological parameters, Astron. Astrophys. 594 (2016) A13 [arXiv:1502.01589] [INSPIRE].

[127] MiniBoonE collaboration, A.A. Aguilar-Arevalo et al., Improved search for $\bar{\nu}_{\mu} \rightarrow \bar{\nu}_{e}$ oscillations in the MiniBooNE experiment, Phys. Rev. Lett. 110 (2013) 161801 [arXiv: 1303.2588] [INSPIRE].

[128] C. Giunti and C. W. Kim, Fundamentals of neutrino physics and astrophysics, Oxford University Press, Oxford U.K. (2007).

[129] Particle Data Group collaboration, K.A. Olive et al., Review of particle physics, Chin. Phys. C 38 (2014) 090001 [INSPIRE].

[130] S.-S. Xue, Diboson channels in ATLAS and CMS experiments, arXiv:1601.06845 [INSPIRE]. 